Eduardo Werley Silva dos Ângelos

\title{
Modelagem Tempo real de Sistemas de Energia Elétrica considerando Sincrofasores e Estimação de Estado Descentralizada
}

Tese apresentada à Escola de Engenharia de São Carlos da Universidade de São Paulo como parte dos requisitos para obtenção do título de Doutor em Ciências, Programa de Engenharia Elétrica.

Área de Concentração: Sistemas Elétricos de Potência.

Orientador: Prof. Dr. Eduardo Nobuhiro Asada.

São Carlos

2013

Trata-se da versão corrigida da tese. A versão original se encontra disponível na EESC/USP, que aloja o Programa de Pós-Graduação de Engenharia Elétrica. 


\begin{abstract}
AUTORIZO A REPRODUÇÃO TOTAL OU PARCIAL DESTE TRABALHO POR QUALQUER MEIO CONVENCIONAL OU ELETRÔNICO, PARA FINS DE ESTUDO E PESQUISA, DESDE QUE CITADA A FONTE.
\end{abstract}

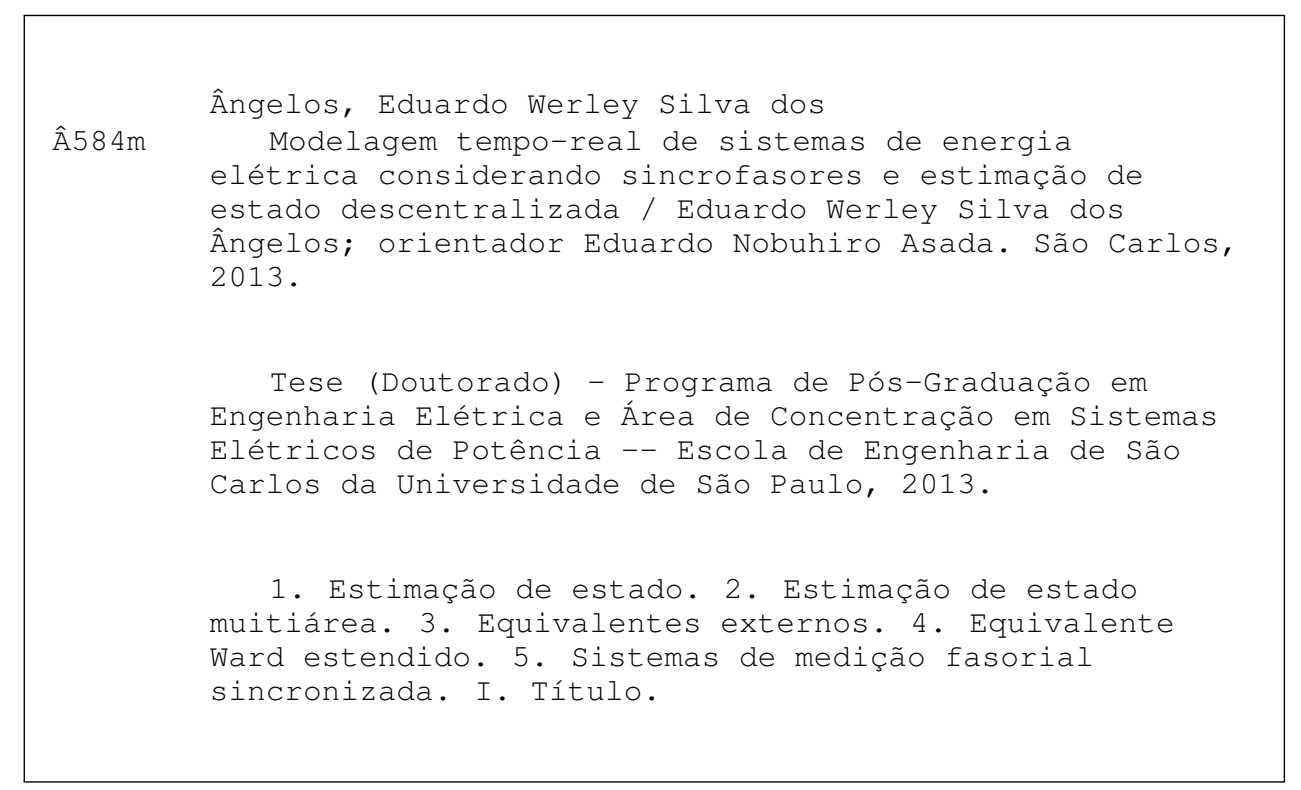


Candidato: Engenheiro EDUARDO WERLEY SILVA DOS ÂNGELOS.

Título da tese: "Modelagem tempo real de sistemas de energia elétrica considerando sincrofasores e estimação de estado descentralizada ".

Data da defesa: 01/11/2013

Comissão Julgadora:

Prof. Dr. Eduardo Nobuhiro Asada (Orientador)

(Escola de Engenharia de São Carlos/EESC)

Prof. Associado João Bosco Augusto London Júnior

(Escola de Engenharia de São Carlos/EESC)

Prof. Dr. Madson Cortes de Almeida

(Universidade Estadual de Campinas/UNICAMP)

Prof. Dr. Júlio César Stacchini de Souza

(Universidade Federal Fluminense/UFF)

Prof. Dr. Antonio José Alves Simões Costa

(Universidade Federal de Santa Catarina/UFSC)
Resultado:
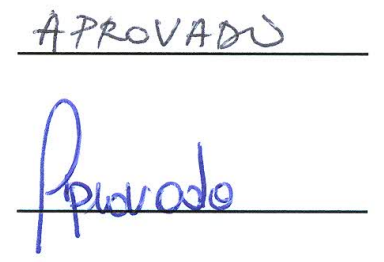

Aprovado

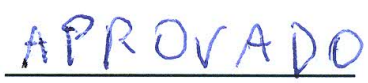

Aprovado

Coordenador do Programa de Pós-Graduação em Engenharia Elétrica e Presidente da Comissão de Pós-Graduação:

Prof. Titular Denis Vinicius Coury 
A minha avó paterna, Josefa, e a Rosa, em memória 


\section{Agradecimentos}

Ao Professor Eduardo Asada, pelos conhecimentos partilhados, pelo profissionalismo, parceria e atenção ao longo destes anos.

A meus pais, Josué e Ana Lúcia, por tornarem este sonho possível.

A minha amiga e esposa Sônia, e nossa pequena Quéren, que veio abrilhantar os intensos anos de doutorado.

A meus familiares e amigos, próximos e distantes.

Aos mestres e colegas da USP São Carlos.

À Universidade de São Paulo e à Escola de Engenharia de São Carlos, pelas instalações e infraestrutura fornecidas para o desenvolvimento desta pesquisa.

Ao Prof. Osvaldo Saavedra e amigos da Engenharia Elétrica da UFMA, que são parte indissolúvel da minha história.

Ao CNPq pelo suporte financeiro. 


\section{Resumo}

Ângelos, E.W.S. Modelagem Tempo real de Sistemas de Energia Elétrica considerando Sincrofasores e Estimação de Estado Descentralizada. 2013. 117 f. Tese (Doutorado em Ciências, Programa de Engenharia Elétrica) - Escola de Engenharia de São Carlos, Universidade de São Paulo, São Carlos, 2013.

Esta tese investiga novas estratégias para a construção de modelos em tempo real de Sistemas Elétricos de Potência. Busca-se a melhoria das funções de Estimação de Estado e aplicações correlatas por meio da consideração da medição fasorial sincronizada, fornecida por dispositivos PMUs, em ambientes onde as regiões monitoradas são de domínios de empresas diferentes e cuja distribuição geográfica apresenta distâncias consideráveis, como é o caso brasileiro. Uma das tarefas mais críticas dentro deste contexto é a representação adequada de sistemas não monitorados, que devem ser modelados de forma precisa, robusta e, preferencialmente, considerando dados que são acessíveis ao operador. A incorporação de redes externas em estimação multiárea é efetuada por uma etapa adicional de estimação ou embutida diretamente nos processos iterativos locais, mediante, neste último caso, a exigência de contínuos fluxos de dados entre áreas. No entanto, constata-se, neste estudo, que modelos clássicos de Equivalentes Externos reduzidos, particularmente os modelos tipo Ward, atendem satisfatoriamente aos requisitos computacionais e de precisão do problema, desde que sejam devidamente atualizados a cada mudança do ponto de operação. Desta forma, considerando sincrofasores de tensão e de corrente coletados por PMUs em regiões de fronteira, desenvolve-se um modelo de Estimação de Estado Descentralizada em que a etapa de pós-processamento por agentes externos independentes é removida, permitindo a obtenção do estado interconectado em um único passo, sem intercâmbio de dados operacionais em tempo real. Dois modelos são implementados, que diferem essencialmente na forma de tratamento dos dados de equivalentes externos. A metodologia é codificada em linguagem $\mathrm{C}++$, sendo validada nos Sistemas IEEE de 14, 30 e 118 barras sob várias configurações de medição e de particionamento, mediante análise estatística e comparação de estimativas com valores de referência. Os resultados obtidos indicam a viabilidade da proposta para o fornecimento de mo- 
delos de estimação de estado mais confiáveis, adaptados à atual tendência de descentralização de redes elétricas, sem grandes alterações nas funções já existentes e sob um custo computacional reduzido. Sugerem também a factibilidade do tratamento conjunto das funções relacionadas a Estimação de Estado e Equivalentes Externos.

Palavras-chave: Estimação de Estado, Estimação de Estado Multiárea, Equivalentes Externos, Equivalente Ward Estendido, Sistemas de Medição Fasorial Sincronizada. 


\section{Abstract}

\section{Ângelos, E.W.S. Power Systems Real-time Modelling with PMUs and Decentralized}

State Estimation. 2013. 117 p. PhD. Thesis - São Carlos Engineering School, University of São Paulo, São Carlos, Brazil, 2013.

New approaches for the real time modelling of Power Systems are investigated in this work. The improvement of State Estimation and related functions is pursued with the aid of synchronized measurements gathered by PMU devices, in a multi-owner environment where utilities are independent and distributed across large distances, as in the Brazilian interconnected system case. One of the critical tasks on this subject is the correct representation of non-monitored networks in precise and feasible way, where less data traffic between operators is preferable. In Multiarea State Estimation, the incorporation of external networks is usually performed as the additional estimation phase or directly included in local estimation models by means of inter-area communication channels. This research shows that classic models of External Equivalents, specially Ward types, meet the computational and precision requirements of the problem if they are correctly updated after changes in the operating point. Thus, by using voltage and current synchrophasors measured by boundary PMUs, a Decentralized State Estimation model is developed, where the need for a post-processing higher coordination step is suppressed, allowing the interconnected state to be found rapidly, in a single step and with no real time data exchange. Two strategies of including on-line information about External Equivalents are proposed, taking it as regular measurements or constraints to be imposed in the classical formulation. A computational software coded in $\mathrm{C}++$ language is built to support the models, which are validated with the IEEE-14, 30 and 118 test bed systems, under several placement strategies and split network schemes. A consistent statistical analysis of the results is also performed, where outcomes are compared with reference values of a regular estimator. Results indicate the feasibility to generate reliable and robust real time models, without significant changes in existing energy management applications, and also shows the greater benefits of integrating State Estimation and External Equivalents into a single framework. 
Keywords: State Estimation, Multiarea State Estimation, External Equivalents, Extended Ward Equivalent. Phasor Measurement Units. 


\section{Lista de Ilustrações}

2.1 Blocos básicos para a construção do modelo de SEPs em tempo real . . . . . . 28

2.2 Modelo $\pi$-ramo unificado . . . . . . . . . . . . . . . . . . . . . . 31

2.3 Arquitetura de um Estimador de Estado Hierárquico . . . . . . . . . . . . . . . 40

2.4 Arquitetura de um Estimador de Estado Distribuído . . . . . . . . . . . . . . . . 40

2.5 Formas de decomposição existentes em ambientes descentralizados . . . . . . . . 41

2.6 Estimação de Estado Multiárea, definições e nomenclaturas . . . . . . . . . . . . 43

2.7 Organização do Sistema no método STLSE . . . . . . . . . . . . . . . . . . . 45

3.1 Convenção para representação de sincrofasores . . . . . . . . . . . . . . . . . 56

3.2 Geração de sincrofasores para um sinal $x(t)$ com frequência distinta da nominal . $\quad 57$

3.3 Blocos funcionais de uma PMU . . . . . . . . . . . . . . . . . . . . . 58

3.4 Medidas disponibilizadas por uma PMU . . . . . . . . . . . . . . . . . . . . 59

4.1 Divisões de um Sistema Elétrico de Potência. . . . . . . . . . . . . . . . . . . . . 64

4.2 Equivalente Externo Ward Linear . . . . . . . . . . . . . . . . . . . . 66

5.1 Organização do sistema multiárea . . . . . . . . . . . . . . . . . . . 72

5.2 Modelagem em tempo real com Equivalentes Externos incorporados ao Estimador. 73

5.3 Redução de Ward em barras de primeira vizinhança . . . . . . . . . . . . . . . 75

5.4 Sistema IEEE-14: configuração das subáreas. . . . . . . . . . . . . . . . . . . . . . . . . . . . . . .

5.5 Sistema IEEE-14: modelo reduzido da área $1 \ldots \ldots$. . . . . . . . . . . . . 81

5.6 Sistema IEEE-14: modelo reduzido da área $2 \ldots \ldots \ldots$. . . . . . . . . . 84

6.1 Sistema IEEE-30: configuração das subáreas . . . . . . . . . . . . . . . . 88

6.2 Sistema IEEE-30: matriz admitância nodal completa vista pela área 1 . . . . . . 90

6.3 Sistema IEEE-30: matriz admitância nodal reduzida, área 1 . . . . . . . . . . . 90

6.4 Sistema IEEE-30: rede completa com equivalentes incorporados . . . . . . . . . . 91

6.5 Erros absolutos de magnitude de tensão na área 2: modelos STLSE, EEEQ e EEEQp 92

6.6 Erros absolutos de ângulo de tensão na área 2: modelos STLSE, EEEQ e EEEQp 92

6.7 Erros absolutos de magnitude de tensão (área 2): modelos EEEQ e EEEQx . . 93

6.8 Erros absolutos de ângulo de tensão (área 2): modelos EEEQ e EEEQx . . . . . 93 
6.9 Sistema IEEE-118: configuração em três subáreas ～. . . . . . . . . . . . . . . . 95

6.10 Sistema IEEE-118 (3 áreas): erros absolutos de magnitude de tensão para o caso 196

6.11 Sistema IEEE-118 (3 áreas): erros absolutos de ângulo de tensão para o caso 1 . 96

6.12 Sistema IEEE-118 (3 áreas): erros absolutos de magnitude de tensão para o caso 297

6.13 Sistema IEEE-118 (3 áreas): erros absolutos de magnitude de tensão para o caso 397

6.14 Sistema IEEE-118: configuração em nove subáreas . . . . . . . . . . . . . . . 100

6.15 Sistema IEEE-118 (9 áreas): erros absolutos de magnitude de tensão . . . . . . 103

6.16 Sistema IEEE-118 (9 áreas): erros absolutos de ângulo de tensão . . . . . . . . . 103 


\section{Lista de Tabelas}

5.1 Sistema IEEE-14: plano de medição da área $1 . \quad \ldots \ldots \ldots$. . . . . . . . . . 81

5.2 Sistema IEEE-14: medidas fornecidas pelas PMUs (área 1) . . . . . . . . . . . . 81

5.3 Sistema IEEE-14: plano de medição da área 2. . . . . . . . . . . . . . . . . 83

5.4 Sistema IEEE-14: medidas fornecidas pelas PMUs (área 2) . . . . . . . . . . . 83

5.5 Comparação dos ângulos estimados (em graus), entre os métodos MQP linear convencional e MQP com equivalentes incorporados. . . . . . . . . . . . . . . . 84

6.1 Desvios-padrão atribuídos para cada tipo de medida . . . . . . . . . . . . . . . 86

6.2 Sistema IEEE-30: partição em duas áreas . . . . . . . . . . . . . . . . . . 88

6.3 Sistema IEEE-118: partição em três áreas. . . . . . . . . . . . . . . . . . . . . . . 95

6.4 Sistemas IEEE-118 (3 áreas): níveis de redundância analisados $(\eta=m / N) \ldots . \quad 95$

6.5 Sistema IEEE-118 (3 áreas): índices $J(x)$ médios observados no caso $3 \ldots \ldots$

6.6 Sistema IEEE-118 (3 áreas): valores estimados e resíduos para o caso 3 . . . . . . 99

6.7 Sistema IEEE-118: partição em nove áreas. . . . . . . . . . . . . . . . . . . . . 101

6.8 Sistema IEEE-118 (9 áreas): tempos computacionais médios (s) em 5000 simulações.101

6.9 Maiores resíduos normalizados por ciclos de estimação e reestimação (caso 1 - erro grosseiro em $\left.P_{19}\right) \ldots \ldots \ldots \ldots$

6.10 Maiores resíduos normalizados (caso 2 - erros grosseiros em $P_{19}$ e $P_{23}$ ) . . . . . 105

6.11 Maiores resíduos normalizados (caso 3 - erros grosseiros em $p_{23-24}$ e $q_{19-34}$ ) . . 105

6.12 Maiores resíduos normalizados (caso 4 -erro grosseiro em $P_{34}^{e q}$ ) . . . . . . . . 105

6.13 Maiores resíduos normalizados (caso 5 - erros grosseiros em $P_{37}^{e q}$ e $P_{38}^{e q}$ ) . . . 105 


\section{Lista de Siglas}

EE

EEDC

EEEQ

EED

EEMA

EEH

EEDR

EEI

EEL

EQE

FCDR

GPS

MQP

PDC

PMU

POO

SCADA

SGE

SEP
Estimação de Estado

Estimação de Estado Descentralizada

Estimação ou Estimador de Estado com Equivalentes Externos incorporados

Estimação de Estado Distribuída

Estimação de Estado Multiárea

Estimação de Estado Hierárquica

Estimador de Estado Desacoplado Rápido

Estimador de Estado Integrado

Estimador de Estado Linearizado

Equivalente Externo

Fluxo de Carga Desacoplado Rápido

Global Positioning System

Mínimos Quadrados Ponderados

Phasor Data Concentrator

Phasor Measurement Unit

Programação Orientada a Objetos

Supervisory Control and Data Acquisition

Sistema de Gerenciamento de Energia

Sistema Elétrico de Potência 
SMFS

STLSE

TC

TP

TVE

UTC

ZHABSE
Sistemas de Medição Fasorial Sincronizada

Standard Two-Level State Estimator

Transformador de Corrente

Transformador de Potencial

Total Vector Error

Universal Time Coordinated

Zhao-Abur Multiarea State Estimator 


\section{Sumário}

1 Introdução $\quad 21$

1.1 Justificativa . . . . . . . . . . . . . . . . . . . . . . . . . . . . . . . 22

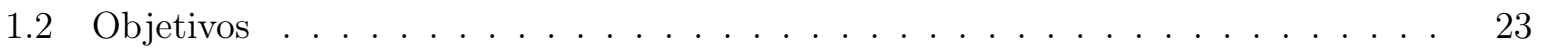

1.3 Organização do Texto . . . . . . . . . . . . . . . . . . . . . . . 24

2 Estimação de Estado em Sistemas Elétricos de Grande Porte 27

2.1 Definição do problema básico . . . . . . . . . . . . . . . . . . . . . . 28

2.1.1 Representação das Medidas . . . . . . . . . . . . . . . . . . . . . 30

2.1.2 Desacoplamento do modelo de medição . . . . . . . . . . . . . . . . . . . . 32

2.1 .3 Modelagem de Restrições de Igualdade . . . . . . . . . . . . . . . . . . . 35

2.1.4 Modelo de medição linearizado . . . . . . . . . . . . . . . . . . . . . . 36

2.1.5 Detecção e Identificação de Erros Grosseiros . . . . . . . . . . . . . . . . . 38

2.2 A Estimação de Estado Multiárea . . . . . . . . . . . . . . . . . . . . . . . . . 39

2.2.1 Formulação do problema . . . . . . . . . . . . . . . . . . . 42

2.2.2 Estimadores Hierárquicos baseados em Duas Etapas . . . . . . . . . . . . 44

2.2.3 Histórico e Trabalhos Correlatos . . . . . . . . . . . . . . . . . . . . 50

2.2.4 Perspectivas e Demandas Atuais . . . . . . . . . . . . . . . . . 53

3 Unidades de Medição Fasorial e Aplicações a Estimação de Estado $\quad 55$

3.1 Definições . . . . . . . . . . . . . . . . . . . . . . . 56

3.1 .1 Medidas disponibilizadas e precisão . . . . . . . . . . . . . . 58

3.2 Sincrofasores no contexto de Estimação de Estado ～. . . . . . . . . . . . . . . . . 60

3.2 .1 Representação das medidas no modelo . . . . . . . . . . . . . . . . . 60

3.2 .2 Aplicação a sistemas interconectados . . . . . . . . . . . . . . . . . . . . . 61

4 Equivalentes Externos em Sistemas Elétricos de Potência $\quad 63$

4.1 Equivalentes Externos do tipo Ward . . . . . . . . . . . . . . . . . . . . . . 64

4.1 .1 Modelo Ward não linear . . . . . . . . . . . . . . . . . . . . . 67

4.1.2 Reação externa e Modelo Ward Estendido . . . . . . . . . . . . . . . . . 68 
5 Estimação de Estado Descentralizada considerando Modelos Externos $\quad 71$

5.1 Definição do modelo das redes externas . . . . . . . . . . . . . . . . . . 73

5.2 Incorporação ao Estimador de Estado . . . . . . . . . . . . . . . . . . . 74

5.2.1 Modelagem como medidas de alta confiança . . . . . . . . . . . . . 75

5.2 .2 Modelagem como Restrições de Igualdade . . . . . . . . . . . . . . . . 77

5.3 Procedimento Geral . . . . . . . . . . . . . . . . . . . 78

5.4 Exemplo Ilustrativo: Sistema IEEE 14 barras com modelo linearizado . . . . . . 79

6 Testes e Validação dos Modelos $\quad 85$

6.1 Sistema IEEE 30 barras . . . . . . . . . . . . . . . . . . . . . . . 87

6.1.1 Configuração do EEEQ com retenção de barras de primeira vizinhança . . 89

$6.1 .2 \quad$ Estado Estimado . . . . . . . . . . . . . . . . . . . . . . . . 89

6.2 Sistema IEEE 118 barras - partição em 3 Áreas . . . . . . . . . . . . . . . . . . . 94

6.3 Sistema IEEE 118 barras - partição em 9 Áreas . . . . . . . . . . . . . . . . . . . 98

6.4 Robustez do modelo frente a erros grosseiros . . . . . . . . . . . . . . . . . 102

$\begin{array}{llr}7 & \text { Conclusões } & 107\end{array}$

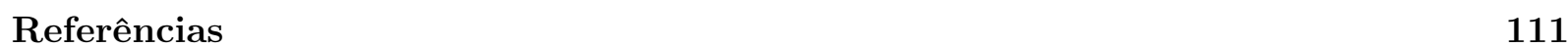




\section{Capítulo 1}

\section{Introdução}

Dentre as funções de análise em tempo real de Sistemas Elétricos de Potência (SEPs), a Estimação de Estado (EE) destaca-se como a base da qual todas as demais dependem para o correto funcionamento. Aliada a aplicações como o Processador Topológico e Análise de Observabilidade, a mesma é responsável pela determinação do estado do sistema, normalmente representado pelas magnitudes e ângulos das tensões nas barras, a partir de um conjunto de medidas redundantes e sujeitas a erros grosseiros.

A recente conjuntura dos SEPs, com sistemas de medição diversificados e estruturas organizacionais desverticalizadas (STOVALL et al., 2006), vem trazendo novos desafios para o controle ótimo e seguro da rede. Há uma demanda crescente nos Sistema de Gerenciamento de Energia (SGE) por modelos em tempo real que sejam mais adaptados à nova dinâmica econômicooperacional presente, caracterizada por companhias e agentes independentes e descentralizados. Aplicativos para a análise de segurança online dentro deste cenário são totalmente dependentes da eficácia do modelo de estimador de estado utilizado (STOTT; ALSAC; MONTICELLI, 1987).

Paralelamente ao crescimento e evolução organizacional das redes elétricas, iniciou-se o estudo da Estimação de Estado Multiárea (EEMA), também conhecida por EE Hierárquica (EEH), Distribuída (EED) ou Descentralizada (EEDC). Seu desenvolvimento foi motivado principalmente pelo surgimento de sistemas interconectados de grande escala nos quais modelos tradicionais de estimação de estado enfrentavam sérias restrições para aplicabilidade (EL-FATTAH; RIBBENSPAVELLA, 1976). Os benefícios diretos decorrentes do uso de modelos multiárea, particularmente enfatizados nos primeiros trabalhos sobre o assunto, são a redução da dimensionalidade do problema global, a redução do tráfego de dados interárea e a diminuição do tempo de processamento (VAN CUTSEM et al., 1980).

Os métodos de EEMA compreendem basicamente os tipos hierárquicos e distribuídos (VAN CUTSEM; RIBBENS-PAVELLA, 1983). Os primeiros são executados em duas etapas de estimação: uma local, representada por um processo de estimação convencional; e outra secundária, 
efetuada por um elemento coordenador independente incumbido da tarefa de resolver as incoerências de estimativas na fronteira, bem como de estabelecer as diferenças entre referências angulares. Os esquemas denominados distribuídos buscam dividir a carga computacional global em processos descentralizados, considerando que áreas adjacentes intercambiam entre si dados de fronteira, de forma que a etapa de coordenação geralmente é ignorada. Neste último caso, é também possível atribuir-se a um operador independente a tarefa de redistribuir determinados resultados obtidos no decorrer do processo iterativo.

\section{$1.1 \quad$ Justificativa}

Um dos principais problemas associados à EEMA é o tratamento de medidas de injeção em barras de fronteira. Nos primeiros trabalhos sobre o tema, especialmente em EEH, tais medidas são simplesmente ignoradas, tanto nos processos locais quanto na etapa de coordenação. A inclusão delas por meio da subtração dos fluxos associados a linhas externas é sugerida em Habiballah (1996). Posteriormente, propôs-se a extensão dos limites de áreas locais a barras de primeira vizinhança, de forma que medidas nestas regiões possam ser aproveitadas nas duas etapas de estimação (ZHAO; ABUR, 2005). Entretanto, neste caso, dados topológicos de linhas próximas às fronteiras devem obrigatoriamente ser enviados ao estimador central.

Constata-se que, em linhas gerais, modelos de EEMA desconsideram redes externas nos processos locais, no sentido que atribuem a um agente independente a tarefa de sincronização e centralização de estimativas comuns. Em EEH, além do fato de uma etapa centralizadora diferir das novas tendências operacionais dos SEPs, a eficácia e o desempenho do próprio elemento central depende também fortemente das estimativas locais. Em EED, quando elementos externos são considerados entre iterações, um tráfego contínuo e elevado de dados interáreas é necessário, condição que ainda é indesejável dentro da dinâmica dos SGEs (STOVALL et al., 2006). Nesse sentido, assevera-se que um modelo em tempo real adequado para as necessidades dos centros de controle modernos deveria, além de ser descentralizado, contemplar uma modelagem externa precisa, de forma a favorecer um pleno desacoplamento interárea e minimizar a transferência de dados entre regiões.

Nos últimos anos, mesmo com a tomada de uma série de medidas de segurança após o grande blackout de 1965 na costa nordeste norte-americana, os SEPs modernos ainda estão sujeitos a graves contingências e interrupções, como as ocorridas em 2003 nos Estados Unidos e Canadá. Este último blecaute teve impacto ainda maior que o primeiro mencionado, tendo sido apontada como uma de suas causas o incorreto funcionamento de um dos Estimadores de Estado locais por haver desconsiderado certas informações sobre a interconexão (U.S. DEPARTMENT OF ENERGY, 2004). Neste caso, uma modelagem imprecisa e a consequente insensibilidade a sistemas externos resultou na total inviabilização do modelo do sistema. 
O estudo de representações de redes elétricas não monitoradas está no âmbito da aplicação Equivalentes Externos. A partir de estimativas do sistema interno, informações topológicas e dados operacionais sobre sistemas externos, torna-se possível para uma rede local construir um modelo externo aproximado, completo ou equivalente, para estudos de contingências e outras funções de análise em tempo real (MONTICELLI et al., 1979; MONTICELLI; GARCIA, 1983). Partindo do pressuposto que o estado de um sistema de grande porte deverá ser obtido por um modelo apropriado de EEMA, nota-se que, por meio das estratégias existentes, é improvável que estimativas adequadas de fronteira sejam obtidas sem a partilha de dados a um agente independente ou sem links diretos de comunicação entre áreas. Sem estas condições, a própria qualidade dos dados fornecidos para o cálculo do equivalente externo então estará comprometida.

A maioria das informações em tempo real processadas por SGEs são oriundas do tradicional Sistema SCADA (do inglês, Supervisory Control and Data Acquisition), onde a varredura e coleta de medidas é executada na ordem de segundos. Atualmente, novos elementos têm sido agregados ao sistema de medição, como os PMUs (do inglês, Phasor Measurement Units), também referenciados por sincrofasores, que formam os chamados Sistemas de Medição Fasorial Sincronizada (SMFS), com taxas de atualização da ordem de milésimos de segundos. Observa-se como tendência mundial a incorporação cada vez maior de sincrofasores aos SEPs. O sistema interligado brasileiro e outras grandes redes elétricas como a norte-americana já possuem iniciativas para a alocação em grande escala de tais medições (MORAES; VOLSKIS; HU, 2008; NASPI, 2011), sendo, portanto, uma tecnologia já em consolidação.

O uso de PMUs em Estimação de Estado vem sendo um problema notoriamente investigado nos últimos anos. Sincrofasores são capazes de fornecer o fasor de tensão complexa nas barras onde são instalados, juntamente com os fasores de corrente dos ramos associados (PHADKE; THORP, 2008). Além disso, como possuem margem para estratégias nativas de calibração e rígidos níveis de padronização (IEEE STD C37.118.1-2011, 2011), assume-se que possuem precisão superior às medidas tratadas pelo sistema SCADA (PHADKE; THORP; KARIMI, 1986; RICE; HEYDT, 2006). No contexto EEMA, um consenso entre os pesquisadores é que a aptidão natural de tais medidas está na tarefa de sincronização entre as áreas, na medida em que possuem como referência angular um sinal de referência global (ZHAO; ABUR, 2005; JIANG; VITTAL; HEYDT, 2008; KORRES, 2011). Estes novos equipamentos também podem ser utilizados para a redução de erros de estimação e para aprimorar o processo de detecção de erros grosseiros, especialmente em regiões de fronteira.

\subsection{Objetivos}

Diante do exposto, esta tese tem como principal objetivo o estudo e desenvolvimento de um modelo de Estimação de Estado Descentralizado que venha a contemplar os novos agentes 
operacionais e sistemas de medição presentes nos SEPs modernos. Considerando as demandas atuais do problema de EEMA, busca-se, dentro da modelagem em tempo real, o aperfeiçoamento das estratégias para a modelagem de redes externas, de forma a permitir a descentralização do modelo global e proporcionar, ao mesmo tempo, estimativas mais precisas e um tráfego de dados interárea reduzido. A possibilidade da construção de modelos clássicos de Equivalentes Externos do tipo Ward e a incorporação deles diretamente nos processos de EE locais é, então, demonstrada, recorrendo-se, para isto, ao uso e alocação de PMUs em barras de fronteiras com vias à atualização dos sistemas equivalentes.

A definição de Equivalentes Externos em tempo real representa uma importante mudança na forma tradicional como esta aplicação vem sendo tratada. Neste sentido, informações atualizadas referentes aos modelos de redes externas estarão disponibilizadas antes da etapa de Estimação de Estado, trazendo implicações interessantes não só para a EEMA, mas para todas as aplicações relacionadas. Faz-se necessário, a priori, o desenvolvimento de uma adequada arquitetura computacional para o tratamento e análise dos modelos desenvolvidos. A filosofia de Programação Orientada a Objetos (POO) mostra-se satisfatória para a modelagem das diferentes estruturas de dados envolvidas no processo.

Em linhas gerais, este estudo possui os seguintes desafios: a compreensão profunda do problema de EEMA e seus maiores desafios, a caracterização correta das informações fornecidas por sincrofasores e sua introdução no problema de estimação de estado e, por último, o estudo da melhor forma de concentrar e de interpretar as diferentes informações obtidas e processadas em diferentes regiões geográficas.

\subsection{Organização do Texto}

Esta tese está estruturada em sete capítulos. Os modelos tratados na metodologia proposta estão definidos em suas respectivas seções, como segue. Prosseguindo-se a esta parte introdutória, no Capítulo 2 aborda-se o problema da Estimação de Estado em SEPs de grande porte. Partindo dos conceitos gerais de EE, discorre-se sobre os fundamentos que levaram ao desenvolvimento dos Estimadores Multiárea e efetua-se a um apanhado geral das principais metodologias e tendências do assunto.

$\mathrm{Na}$ terceira parte do texto, investigam-se as Unidades de Medição Fasorial, abordando as definições e padronizações associadas aos dispositivos e caracterizando as medidas que são disponibilizadas. São revisadas também as formas de integração destes equipamentos aos modelos de EE clássica e multiárea.

O Capítulo 4 compreende o estudo de Equivalentes Externos e sua relevância no processo de construção do modelo de SEPs em tempo real. Enfatizam-se os modelos mais difundidos em SGEs e aqueles que podem ser mais facilmente integrados a aplicações de análise online. 
A definição da metodologia para EEDC objeto da tese é então apresentada no Capítulo 5. Dois modelos para EEMA são propostos, cada um representando uma forma específica de tratamento matemático das informações relacionadas a Equivalentes Externos. Nesta mesma seção apresentam-se também um algoritmo geral e um exemplo de aplicação da metodologia ao Sistema IEEE de 14 barras.

No Capítulo 6, são apresentados os testes efetuados nos modelos propostos, nos sistemas IEEE de 30 e 118 barras. A arquitetura computacional de suporte aos métodos também é tratada brevemente. As análises são realizadas principalmente sob as óticas de precisão, desempenho computacional e robustez frente a erros. São também consideradas, para fins de comparação, outras importantes abordagens presentes na literatura técnica.

Por fim, no Capítulo 7, são tecidas as conclusões acerca da pesquisa. Ressaltam-se as maiores contribuições dos modelos propostos, suas perspectivas e desdobramentos no campo de estudo. 


\section{Capítulo 2}

\section{Estimação de Estado em Sistemas Elétricos de Grande Porte}

A Estimação de Estado (EE) compõe o conjunto de aplicativos responsáveis pela construção do modelo das redes elétricas em tempo real. Originou-se da necessidade de um controle e monitoramento mais efetivo dos sistemas, sobretudo para fins de análise de segurança. Seu estabelecimento foi acompanhado e motivado também pela maturação das diversas áreas de engenharia e computação envolvidas, como instrumentação, sistemas de comunicação, de processamento e armazenamento de dados.

Os blocos típicos para a construção do modelo de um SEP em tempo real, presentes nos Sistema de Gerenciamento de Energia, estão ilustrados na Fig. (2.1). Os dados processados incluem medidas analógicas, como fluxos e injeções de potência, e digitais, associadas ao estado (ligado ou desligado) de chaves e disjuntores, que são coletadas pelo Sistema SCADA e atualizadas na ordem de minutos. Novos sistemas de aquisição, como os concentradores de dados de PMUs, também já estão presentes em vários centros de controle, provendo medidas fasoriais de tensão e corrente sincronizadas na ordem de microssegundos.

A representação da rede é, normalmente, definida em dois passos: obtenção da topologia atual e cálculo do estado. A primeira etapa, sob responsabilidade da aplicação denominada processador topológico, tem por objetivo determinar o modelo da rede em termos de barras e ramos (modelo barra-ramo), ou diagrama unifilar, considerando parâmetros armazenados em banco de dados e informações correntes sobre as condições de chaves e outros dispositivos. Nesta mesma etapa é também efetuada a alocação de medidores. Após a obtenção da topologia atual da rede, as demais medidas são então processadas pelo Estimador de Estado, que busca encontrar a melhor estimativa para o estado atual do sistema, comumente representado pelas magnitudes e ângulos das tensões 


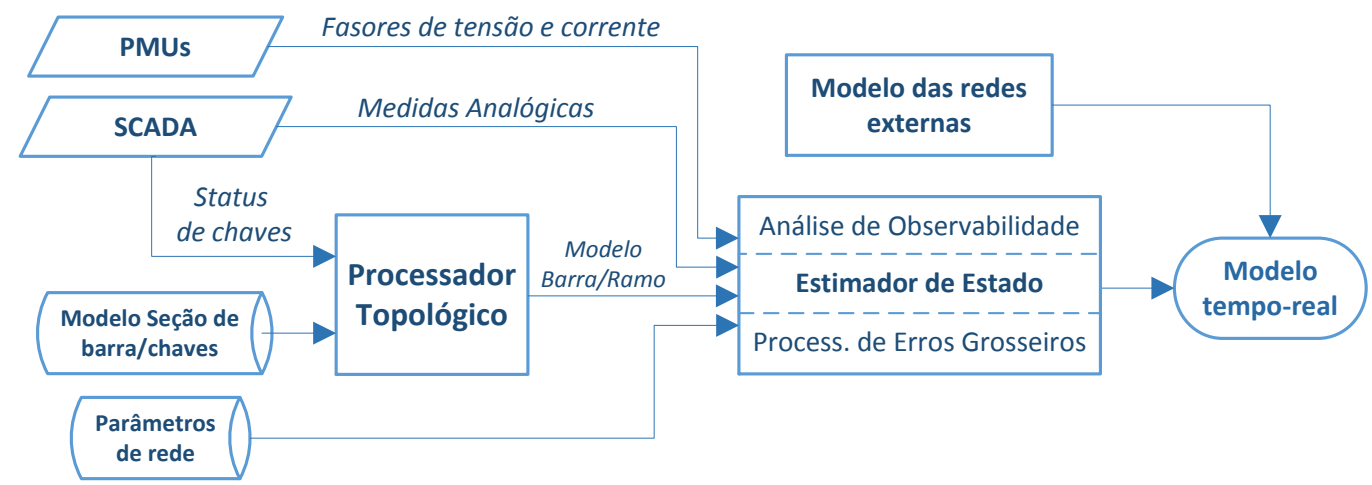

Figura 2.1 - Blocos básicos para a construção do modelo de SEPs em tempo real

nas barras. No modelo de Estimação Generalizada, estas duas fases podem ser processadas em um único passo, entretanto, o custo computacional associado impõe alguns fatores limitantes para sua aplicação.

Quando o conjunto de medidas considerado é suficiente para se calcular o estado, o sistema é dito observável. A análise de observabilidade será requerida cada vez que houver alteração na topologia ou no conjunto de medidas analógicas. Normalmente, o plano de medição de um SEP é disposto de forma a garantir não somente a observabilidade, mas a redundância do sistema. Logo, o estimador de estado atua também como um filtro, de modo a eliminar as medidas com comportamento mais discrepante. A função dedicada Processamento de Erros Grosseiros é executada após o cálculo do estado, sendo que as medidas errôneas são descartadas ou corrigidas, até que não sejam detectados mais erros.

Para finalizar a construção do modelo em tempo real, é necessária também a representação das redes externas. Embora não sejam de interesse direto, seus efeitos manifestam-se sobretudo na ocorrência de contingências na rede local, como faltas, saída de linhas ou perda de geração. Uma vez obtido o modelo da rede, dele dependerá todas as demais funções de análise e controle em tempo real, como o Fluxo de Potência Ótimo com Restrições de Segurança e a Análise de Contingências.

As demais Seções deste capítulo buscam detalhar especificamente a função Estimador de Estado, discorrendo sobre o modelo matemático correspondente e, posteriormente, expandido o conceito básico para SEPs de dimensões elevadas, assunto principal desta tese.

\subsection{Definição do problema básico}

A modelagem matemática do problema de EE é determinada pelo chamado modelo de medição, que expressa a correlação entre um dado conjunto de $m$ medidas coletadas ao longo de um 
sistema elétrico com $n_{b}$ barras, os erros de medição associados a elas e as variáveis de estado a serem determinadas. Schweppe, em seus trabalhos pioneiros no assunto (SCHWEPPE; WILDES, 1970; SCHWEPPE; ROM, 1970; SCHWEPPE, 1970), equacionou o problema de estimação de estado da seguinte forma:

$$
\mathbf{z}=\mathbf{h}(\mathbf{x})+\mathbf{e}
$$

em que $\mathbf{h}$ é o vetor de $m$ equações não lineares relacionando o vetor de estado verdadeiro $\mathbf{x}$, de dimensão $n=2 n_{b}-1(n<m)$, ao vetor de medidas $\mathbf{z}$ e ao vetor dos erros aleatórios das medidas e com distribuição normal.

Costuma-se definir como estado do sistema as magnitudes e ângulos das tensões nas barras. Na formulação clássica, o ângulo de tensão na barra de referência é excluído do vetor de estado, ao qual normalmente atribui-se o valor $0^{\circ}$. Por outro lado, o vetor de medidas é normalmente composto por fluxos, injeções de potência, magnitudes de corrente, magnitudes de tensão e, eventualmente, fasores sincronizados de tensão e corrente originados de PMUs. Considera-se que o vetor de erros aleatórios e possui distribuição Gaussiana com média zero e matriz covariância $\mathbf{R}$, isto é, $E(\mathbf{e})=0$ e $E\left(\mathbf{e e}^{\prime}\right)=\mathbf{R}$. Assume-se também que os erros são não correlacionados, com variâncias atribuídas de acordo com a precisão de cada medidor.

Dentre as técnicas para se resolver o sistema sobredeterminado representado pela Eq. (2.1), a mais difundida é a abordagem por Mínimos Quadrados Ponderados (MQP), onde o estado estimado $\hat{x}$ é obtido através da minimização da seguinte função objetivo:

$$
J(\hat{\mathbf{x}})=\frac{1}{2}[\mathbf{z}-\mathbf{h}(\hat{\mathbf{x}})]^{\prime} \mathbf{R}^{-1}[\mathbf{z}-\mathbf{h}(\hat{\mathbf{x}})]
$$

A primeira condição de otimalidade associada ao índice de desempenho é dada por:

$$
\left.\frac{\partial J}{\partial \mathbf{x}}\right|_{\mathbf{x}=\hat{\mathbf{x}}}=\mathbf{0} \quad \Rightarrow \quad \mathbf{H}^{\prime}(\hat{\mathbf{x}}) \mathbf{R}^{-1}[\mathbf{z}-\mathbf{h}(\hat{\mathbf{x}})]=\mathbf{0}
$$

em que $\mathbf{H}(\hat{\mathbf{x}})=\left.\frac{\partial \mathbf{h}}{\partial \mathbf{x}}\right|_{\mathbf{x}=\hat{\mathbf{x}}}$ é a matriz Jacobiana de $\mathbf{h}(\mathbf{x})$ calculada no ponto $\hat{\mathbf{x}}$. O estado estimado pode ser obtido por intermédio das Equações Normais de Gauss-Newton, considerando o seguinte processo iterativo:

$$
\begin{aligned}
\mathbf{G}\left(\hat{\mathbf{x}}^{v}\right) \boldsymbol{\Delta} \mathbf{x}^{v} & =\mathbf{H}^{\prime}\left(\hat{\mathbf{x}}^{v}\right) \mathbf{R}^{-1} \mathbf{r}\left(\hat{\mathbf{x}}^{v}\right) \\
\hat{\mathbf{x}}^{v+1} & =\hat{\mathbf{x}}^{v}+\Delta \mathbf{x}^{v}
\end{aligned}
$$

onde $\mathbf{G}\left(\hat{\mathbf{x}}^{v}\right)=\mathbf{H}^{\prime}\left(\hat{\mathbf{x}}^{v}\right) \mathbf{R}^{-1} \mathbf{H}\left(\hat{\mathbf{x}}^{v}\right)$ é conhecida por Matriz Ganho e $\mathbf{r}\left(\hat{\mathbf{x}}^{v}\right)=\mathbf{z}-\mathbf{h}\left(\hat{\mathbf{x}}^{v}\right)$ é o vetor de resíduos de estimação. O vetor $\boldsymbol{\Delta} \mathbf{x}^{v}$ representa a correção a ser aplicada ao estado na iteração $v$. 
Nos casos em que há escassez de medidas $(m<n)$, o problema de EE torna-se indeterminado $^{1}$, podendo ser formulado em termos da Mínima Norma. Em de Almeida, Garcia e Asada (2012), as soluções por mínimos quadrados ou mínima norma são partes de um única formulação multiobjetivo, resultando no chamado modelo de EE Regularizado.

A factibilidade matemática do estimador de estado diante da quantidade e localização das medidas disponíveis é definida pela análise de observabilidade. Duas principais classes estão representadas neste contexto, os métodos numéricos e os topológicos. Nos primeiros, a viabilidade do problema de EE é determinada a partir de informações sobre a topologia da rede e a configuração de medidores, evitando assim a necessidade de cálculos para a determinação do posto da Matriz Ganho (KRUMPHOLZ; CLEMENTS; DAVIS, 1980; QUINTANA; SIMÕES-COSTA; MANDEL, 1982). Já os métodos numéricos podem estar baseados na fatoração triangular da própria matriz Ganho, como proposto em Monticelli e Wu (1985b), na fatoração da matriz Jacobiana sem a necessidade da solução de sistemas lineares (LONDON; ALBERTO; BRETAS, 2007), ou ainda na chamada Matriz de Gram (DE ALMEIDA; ASADA; GARCIA, 2008). Este assunto e outros correlatos são amplamente investigados em Monticelli (1999).

\subsubsection{Representação das Medidas}

No que segue, descreve-se a formação do vetor $\mathbf{h}(\mathbf{x})$ para os tipos de medidas mais comuns, assumindo como variáveis de estado as magnitudes e ângulos das tensões nas barras.

- Fluxos de Potência $\left(P_{k m}, P_{m k}, Q_{k m}, Q_{m k}\right)$

Considerando o modelo $\pi$ de ramo unificado mostrado na Figura (2.2), que representa uma linha de transmissão ou transformador conectado entre as barras $k$ e $m$ em termos da admitância série $\left(y_{k m}\right)$, admitâncias shunt $\left(y_{k m}^{s h}, y_{m k}^{s h}\right)$, taps $\left(a_{k m}, a_{m k}\right)$ e elementos defasadores $\left(\varphi_{k m}, \varphi_{m k}\right)$, as expressões gerais dos fluxos serão dadas por:

$$
\begin{array}{r}
P_{k m}=\left(a_{k m} V_{k}\right)^{2} g_{k m}-a_{k m} V_{k} a_{m k} V_{m}\left(g_{k m} \cos \gamma_{k m}+b_{k m} \operatorname{sen} \gamma_{k m}\right) \\
P_{m k}=V_{m}^{2} g_{k m}-a_{k m} V_{k} a_{m k} V_{m}\left(g_{k m} \cos \gamma_{k m}-b_{k m} \operatorname{sen} \gamma_{k m}\right) \\
Q_{k m}=-\left(a_{k m} V_{k}\right)^{2}\left(b_{k m}+b_{k m}^{s h}\right)-a_{k m} V_{k} a_{m k} V_{m}\left(g_{k m} \operatorname{sen} \gamma_{k m}-b_{k m} \cos \gamma_{k m}\right) \\
Q_{m k}=-V_{m}^{2}\left(b_{k m}+b_{k m}^{s h}\right)+a_{k m} V_{k} a_{m k} V_{m}\left(g_{k m} \operatorname{sen} \gamma_{k m}+b_{k m} \cos \gamma_{k m}\right)
\end{array}
$$

\footnotetext{
${ }^{1}$ Condição necessária mas não suficiente.
} 


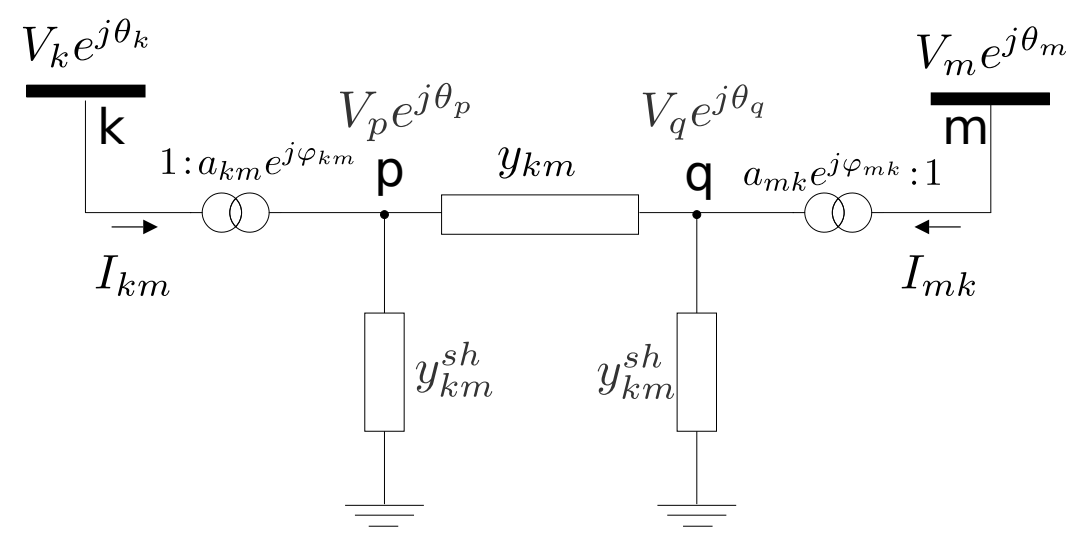

Figura 2.2 - Modelo $\pi$ - ramo unificado

em que $\gamma_{k m}=\theta_{k m}+\varphi_{k m}+\varphi_{m k}$. Para linhas de transmissão, $a_{k m}=a_{m k}=1$ e $\varphi_{k m}=\varphi_{m k}=0$. Para transformadores em fase com tap na barra $k, y_{k m}^{s h}=y_{m k}^{s h}=0, \varphi_{k m}=\varphi_{m k}=0$ e $a_{m k}=1$. Já no caso de defasadores puros com atuação nessa mesma barra, $y_{k m}^{s h}=y_{m k}^{s h}=0, a_{k m}=a_{m k}=1$ e $\varphi_{m k}=0$.

- Injeções de Potência $\left(P_{k}, Q_{k}\right)$

Medidas de injeção de potência ativa e reativa, em uma dada barra $k$, podem ser expressas por:

$$
\begin{aligned}
P_{k} & =V_{k} \sum_{m \in K} V_{m}\left(G_{k m} \cos \theta_{k m}+B_{k m} \operatorname{sen} \theta_{k m}\right) \\
Q_{k} & =V_{k} \sum_{m \in K} V_{m}\left(G_{k m} \cos \theta_{k m}-B_{k m} \operatorname{sen} \theta_{k m}\right)
\end{aligned}
$$

onde $m$ pertence ao conjunto de barras vizinhas ao nó $k$, e $Y_{k m}=G_{k m}+j B_{k m}$ é o elemento da matriz admitância nodal $(\mathbf{Y}=\mathbf{G}+j \mathbf{B})$ na posição $\mathrm{km}$.

- Magnitudes de Corrente $\left(\left|I_{k m}\right|,\left|I_{m k}\right|\right)$

Medidas desta categoria podem ser diretamente incluídas no problema de EE considerando as seguintes relações: 


$$
\begin{aligned}
\left|I_{k m}\right| & =\frac{\left(P_{k m}^{2}+Q_{k m}^{2}\right)^{1 / 2}}{V_{k}} \\
\left|I_{m k}\right| & =\frac{\left(P_{m k}^{2}+Q_{m k}^{2}\right)^{1 / 2}}{V_{m}}
\end{aligned}
$$

em que $\left|I_{k m}\right|$ e $\left|I_{m k}\right|$, são as magnitudes das correntes complexas representadas na Fig. (2.2.)

- Medidas relacionadas ao estado $\left(V_{k}^{\text {med. }}, \theta_{k}^{\text {med. }}\right)$

Neste conjunto estão inclusas magnitudes $\left(V_{k}^{\text {med. }}\right)$ e ângulos $\left(\theta_{k}^{\text {med. }}\right)$ de tensão, associados a uma dada barra $k$. Como são linearmente relacionadas ao estado, seus componentes na matriz Jacobiana são unitários. Em termos de modelo de medição, estas medidas estarão relacionadas ao estado verdadeiro da barra $k\left(V_{k} \angle \theta_{k}\right)$ da seguinte forma:

$$
\begin{gathered}
V_{k}^{\text {med. }}=V_{k}+e_{v} \\
\theta_{k}^{\text {med. }}=\theta_{k}+e_{\theta}
\end{gathered}
$$

em que $e_{v}$ e $e_{\theta}$ são os erros associados a cada medida.

Medidas de ângulo devem ser incorporadas ao problema com cautela, de forma que não estejam em conflito com a referência angular interna do sistema. Em redes interconectadas, esta questão torna-se sobremaneira relevante, como será mostrado nas próximas seções.

\subsubsection{Desacoplamento do modelo de medição}

Assumindo que o vetor de medidas $\mathbf{z}$ seja redefinido da seguinte forma:

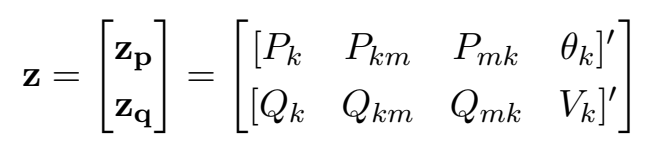

onde $\mathbf{z}_{\mathbf{p}}$ é o vetor de dimensão $m_{p}$ formado por medidas de injeção ativa, fluxos de potência ativa e ângulos de tensão; e $\mathbf{z}_{\mathbf{q}}$ é o vetor de dimensão $m_{q}$ composto de medidas de injeção reativa, fluxos de potência reativa e magnitudes de tensão.

Adicionalmente, suponha também que o vetor de estado $\mathrm{x}$ seja particionado como segue:

$$
\mathbf{x}=\left[\begin{array}{ll}
\theta^{\prime} & \mathbf{V}^{\prime}
\end{array}\right]^{\prime}
$$


em que $\mathbf{V}$ é o vetor de dimensão $n_{b}$ cujos elementos são as magnitudes das tensões de barras, e $\boldsymbol{\theta}$, de dimensão $n b-1$, é o vetor contendo os ângulos das tensões nodais.

Sob estas considerações, o modelo de medição representado na Eq. (2.1) poderá ser reescrito da seguinte forma:

$$
\left\{\begin{array}{l}
\mathbf{z}_{\mathbf{p}}=\mathbf{h}_{\mathbf{p}}(\mathbf{x})+\mathbf{e}_{\mathbf{p}} \\
\mathbf{z}_{\mathbf{q}}=\mathbf{h}_{\mathbf{q}}(\mathbf{x})+\mathbf{e}_{\mathbf{q}}
\end{array}\right.
$$

em que os subscritos $p$ e $q$ redefinem as mesmas grandezas apresentadas anteriormente nos subproblemas ativo e reativo. Considera-se também que $E\left(\mathbf{e}_{\mathbf{p}}\right)=0, E\left(\mathbf{e}_{\mathbf{p}} \mathbf{e}_{\mathbf{p}}^{\prime}\right)=\mathbf{R}_{\mathbf{p}}$ e que $E\left(\mathbf{e}_{\mathbf{q}}\right)=0, E\left(\mathbf{e}_{\mathbf{q}} \mathbf{e}_{\mathbf{q}}^{\prime}\right)=\mathbf{R}_{\mathbf{q}}$.

A Equação Normal de Gauss correspondente ao novo problema será, então, dada por

$$
\left[\begin{array}{cc}
\mathbf{A}_{\mathbf{p} \theta} & \mathbf{A}_{\mathbf{p v}} \\
\mathbf{A}_{\mathbf{q} \theta} & \mathbf{A}_{\mathbf{q v}}
\end{array}\right]\left[\begin{array}{c}
\Delta \theta \\
\Delta \mathbf{V}
\end{array}\right]=\left[\begin{array}{cc}
\mathbf{H}_{\mathbf{p} \theta} & \mathbf{H}_{\mathbf{p v}} \\
\mathbf{H}_{\mathbf{q} \theta} & \mathbf{H}_{\mathbf{q v}}
\end{array}\right]^{\prime}\left[\begin{array}{cc}
\mathbf{R}_{\mathbf{p}} & 0 \\
0 & \mathbf{R}_{\mathbf{q}}
\end{array}\right]^{-1}\left[\begin{array}{c}
\mathbf{r}_{\mathbf{p}} \\
\mathbf{r}_{\mathbf{q}}
\end{array}\right]
$$

em que

$$
\left[\begin{array}{cc}
\mathbf{A}_{\mathbf{p} \theta} & \mathbf{A}_{\mathbf{p v}} \\
\mathbf{A}_{\mathbf{q} \theta} & \mathbf{A}_{\mathbf{q v}}
\end{array}\right]=\left(\left[\begin{array}{ll}
\mathbf{H}_{\mathbf{p} \theta} & \mathbf{H}_{\mathbf{p v}} \\
\mathbf{H}_{\mathbf{q} \theta} & \mathbf{H}_{\mathbf{q v}}
\end{array}\right]^{\prime}\left[\begin{array}{cc}
\mathbf{R}_{\mathbf{p}} & 0 \\
0 & \mathbf{R}_{\mathbf{q}}
\end{array}\right]^{-1}\left[\begin{array}{ll}
\mathbf{H}_{\mathbf{p} \theta} & \mathbf{H}_{\mathbf{p v}} \\
\mathbf{H}_{\mathbf{q} \theta} & \mathbf{H}_{\mathbf{q v}}
\end{array}\right]\right)
$$

As submatrizes $\mathbf{H}_{\mathbf{p} \theta}, \mathbf{H}_{\mathbf{p v}}$ e $\mathbf{H}_{\mathbf{q} \theta}, \mathbf{H}_{\mathbf{q v}}$ denotam, respectivamente, as derivadas das funções não lineares dos problemas ativo $\left(\mathbf{h}_{\mathbf{p}}(\mathbf{x})\right)$ e reativo $\left(\mathbf{h}_{\mathbf{q}}(\mathbf{x})\right)$ em relação aos ângulos e magnitudes das tensões complexas nas barras. As matrizes covariâncias dos erros de medição associados aos vetores $\mathbf{z}_{\mathbf{p}}$ e $\mathbf{z}_{\mathbf{q}}$ estão representadas em $\mathbf{R}_{\mathbf{p}}$ e $\mathbf{R}_{\mathbf{q}}$, enquanto $\mathbf{r}_{\mathbf{p}}$ e $\mathbf{r}_{\mathbf{q}}$ denotam os vetores de resíduos relacionados aos dois subproblemas.

O desacoplamento do problema de EE consiste em se aplicar ao modelo de medição as mesmas aproximações feitas no problema do Fluxo de Carga Desacoplado Rápido (FCDR) (STOTT; ALSAC, 1974; DECKMANN et al., 1980a; AMERONGEN, 1989), onde as sensibilidades do problema ativo em relação às magnitudes de tensão (sensibilidades $P-V$ ) e do problema reativo em relação aos ângulos (sensibilidades $Q-\theta$ ) são desprezadas. As alterações envolvem ainda a inicialização das variáveis de estado em flat start $(V=1 p$.u. e $\theta=0)$ e o uso de matrizes constantes no processo iterativo. Sob estas circunstâncias, a Eq. (2.18) pode ser dividida em dois subproblemas:

$$
\begin{aligned}
\mathbf{A}_{\mathbf{p} \theta} \Delta \boldsymbol{\theta} & =\mathbf{H}_{\mathbf{p} \theta}^{\prime} \mathbf{R}_{\mathbf{p}}{ }^{-1} \mathbf{r}_{\mathbf{p}} \\
\mathbf{A}_{\mathbf{q v}} \Delta \mathbf{V} & =\mathbf{H}_{\mathbf{q v}}^{\prime} \mathbf{R}_{\mathbf{q}}{ }^{-1} \mathbf{r}_{\mathbf{q}}
\end{aligned}
$$


Caso as aproximações sejam aplicadas somente nas matrizes $\mathbf{A}_{\mathbf{p} \theta}$ e $\mathbf{A}_{\mathbf{q v}}$, ocorre o chamado desacoplamento no algoritmo (HORISBERGER; RICHARD; ROSSIER, 1976), onde a mesma solução do problema completo (acoplado) é alcançada, mas em um maior número de iterações. No entanto, esta estratégia apresenta falhas sobretudo em sistemas com elevada razão $\frac{X}{R}$.

Os algoritmos que operam o chamado desacoplamento no modelo buscam sanar estas deficiências aplicando as aproximações também na matriz Jacobiana. Neste caso, a solução final pode diferir da alcançada no modelo completo, todavia é possível mostrar que os erros apresentados são aceitáveis para o problema de EE (GARCIA; MONTICELLI; ABREU, 1979). Nesse contexto, destacam-se os modelos propostos por Allemong, Radu e Sasson (1982) e por Monticelli e Garcia (1990). Neste último, efetua-se um estudo aprofundado sobre o problema do desacoplamento em estimadores de estado e as diversas aproximações envolvidas. Mostra-se também que o desacoplamento no modelo é uma propriedade inerente dos sistemas, sob certas condições, podendo ser estabelecido sem maiores modificações na estrutura do modelo original. Por fim, no mesmo trabalho é desenvolvido o algoritmo do chamado Estimador de Estado Desacoplado Rápido (EEDR), que se tornou uma das referências em aplicações práticas, descrito resumidamente a seguir:

\section{Algoritmo - Estimador Desacoplado Rápido Versão BX}

1. Cálculo da correção de $\boldsymbol{\theta}(1 / 2$ iteração ativa)

$$
\begin{aligned}
\widetilde{\mathbf{A}}_{\mathbf{p} \theta} \boldsymbol{\Delta} \boldsymbol{\theta}^{v} & =\mathbf{H}_{\mathbf{p} \theta}^{\prime} \mathbf{R}_{\mathbf{p}}^{-1} \mathbf{r}_{\mathbf{p}}\left(\mathbf{V}^{v}, \boldsymbol{\theta}^{v}\right) \\
\boldsymbol{\theta}^{v+1} & =\boldsymbol{\theta}^{v}+\boldsymbol{\Delta} \boldsymbol{\theta}^{v}
\end{aligned}
$$

2. Cálculo da correção de $\mathbf{V}$ (1/2 iteração reativa)

$$
\begin{aligned}
\widetilde{\mathbf{A}}_{\mathbf{q v}} \Delta \mathbf{V}^{v} & =\mathbf{H}_{\mathbf{q v}}^{\prime} \mathbf{R}_{\mathbf{q}}^{-\mathbf{1}} \mathbf{r}_{\mathbf{q}}\left(\mathbf{V}^{v}, \boldsymbol{\theta}^{v+1}\right) \\
\mathbf{V}^{v+1} & =\mathbf{V}^{v}+\Delta \mathbf{V}^{v}
\end{aligned}
$$

- Obs: As matrizes Jacobianas devem ser calculadas em flat start. Para o cálculo de $\mathbf{H}_{\mathbf{q v}}$, as susceptâncias $b_{k m}$ são substituídas por $-1 / x_{k m}$. Em $\mathbf{H}_{\mathbf{p} \theta}$, medidas de fluxos e injeções de potência devem ser normalizadas pelas tensões nas barras correspondentes. Os sobrescritos nas matrizes $\mathbf{A}_{\mathbf{p} \theta}$ e $\mathbf{A}_{\mathbf{q v}}$ indicam que as mesmas deverão ser obtidas considerando as aproximações anteriores. 


\subsubsection{Modelagem de Restrições de Igualdade}

Além dos tipos de medidas já abordados, outros tipos de informações devem ser tratadas pelos Estimadores de Estado, como as pseudomedidas, que incluem dados sobre despacho de geradores, potência demandada em subestações ou outros dados históricos, utilizados, principalmente, para se garantir a observabilidade do sistema. Importantes aspectos relacionados à incorporação de informações a priori em Estimadores de estado são discutidos em Do Coutto Filho e Souza (2009) e Do Coutto Filho, Souza e Freund (2009). Há também as chamadas medidas virtuais, associadas a informações que normalmente não requerem monitoramento, como injeções nulas em barras de transferência.

Uma forma de se incorporar tais informações é integrando-as como medidas de baixa confiança, no caso das pseudomedidas, ou de alta confiança, no caso das últimas mencionadas. Embora muito utilizada, esta estratégia pode trazer instabilidades numéricas para o problema de EE, particularmente relacionadas à matriz Ganho. Dentre as soluções para se contornar este problema, pode-se destacar o uso de modelos que evitam a formação da matriz Ganho, como o método da Matriz Aumentada (GJELSVIK; AAM; HOLTEN, 1985), a aplicação de transformação ortogonal (SIMÕES-COSTA; QUINTANA, 1981a; SIMÕES-COSTA; QUINTANA, 1981b) e a utilização de restrições de igualdade. Esta última estratégia, inicialmente proposta em Aschmoneit, Peterson e Adrian (1977) é descrita a seguir.

O problema de MQP com restrições de igualdade pode ser formulado como:

$$
\begin{array}{ll}
\text { Minimizar } & J(\mathbf{x}) \\
\text { sujeito a } & \mathbf{c}(\mathbf{x})=0
\end{array}
$$

onde $\mathbf{c}(\mathbf{x})$ representa o vetor de equações não lineares associadas às restrições de igualdade, de dimensão $n_{c}$. A função Lagrangeana correspondente ao problema é dada por:

$$
\mathcal{L}(\mathbf{x}, \boldsymbol{\lambda})=\frac{1}{2}[\mathbf{z}-\mathbf{h}(\mathbf{x})]^{\prime} \mathbf{R}^{-1}[\mathbf{z}-\mathbf{h}(\mathbf{x})]+\boldsymbol{\lambda}^{\prime} \mathbf{c}(\mathbf{x})
$$

onde $\boldsymbol{\lambda}\left(n_{c} \times 1\right)$ é o vetor dos multiplicadores de Lagrange associados às restrições. Aplicando-se a primeira condição de otimalidade à Eq. (2.27), tem-se que:

$$
\begin{array}{rll}
\partial \mathcal{L} / \partial \mathbf{x}=\mathbf{0} & \Rightarrow & \mathbf{H}^{\prime}(\mathbf{x}) \mathbf{R}^{-1}[\mathbf{z}-\mathbf{h}(\mathbf{x})]-\mathbf{C}^{\prime}(\mathbf{x}) \boldsymbol{\lambda}=\mathbf{0} \\
\partial \mathcal{L} / \boldsymbol{\lambda}=\mathbf{0} & \Rightarrow & -\mathbf{c}(\mathbf{x})=\mathbf{0}
\end{array}
$$

onde $\mathbf{H}(\mathbf{x})=\partial \mathbf{h}(\mathbf{x}) / \partial \mathbf{x}$ e $\mathbf{C}(\mathbf{x})=\partial \mathbf{c}(\mathbf{x}) / \partial \mathbf{x}$. Finalmente, a solução ótima pode ser obtida 
utilizando o método de Gauss-Newton, considerando o seguinte processo iterativo:

$$
\left[\begin{array}{cc}
\mathbf{H}^{\prime}\left(\hat{\mathbf{x}}^{v}\right) \mathbf{R}^{-1} \mathbf{H}\left(\hat{\mathbf{x}}^{v}\right) & -\mathbf{C}^{\prime}\left(\mathbf{x}^{v}\right) \\
-\mathbf{C}\left(\mathbf{x}^{v}\right) & \mathbf{0}
\end{array}\right]\left[\begin{array}{c}
\Delta \mathbf{x} \\
\boldsymbol{\lambda}^{v+1}
\end{array}\right]=\left[\begin{array}{c}
\mathbf{H}^{\prime}\left(\mathbf{x}^{v}\right) \mathbf{R}^{-1} \mathbf{r}\left(\mathbf{x}^{v}\right) \\
\mathbf{c}\left(\mathbf{x}^{v}\right)
\end{array}\right]
$$

onde $\boldsymbol{\Delta} \mathbf{x}$ é a correção a ser aplicada ao estado na iteração $v$.

Com esta formulação, as restrições de igualdade passarão a compor um problema separado das medidas convencionais. Como consequência, o produto matricial sobre o vetor c é evitado. No entanto, providências adicionais devem ser tomadas para se efetuar a fatoração da matriz de coeficientes, que passa a ser do tipo indefinida.

O relacionamento entre a abordagem por restrições de igualdade e o uso de pesos elevados na formulação básica do método MQP pode ser demonstrado da seguinte forma. Suponha que o índice $J(x)$ (Eq. 2.2) seja reescrito de forma que medidas virtuais estejam separadas das regulares, ou seja:

$$
J(\mathbf{x})=\frac{1}{2}[\mathbf{z}-\mathbf{h}(\mathbf{x})]^{\prime} \mathbf{R}^{-1}[\mathbf{z}-\mathbf{h}(\mathbf{x})]+\frac{\rho}{2} \mathbf{c}(\mathbf{x})^{\prime} \mathbf{c}(\mathbf{x})
$$

onde $\rho$ é um fator de escala associado às medidas virtuais, de grandeza muito maior que os pesos das medidas tradicionais. As primeiras condições de otimalidade para este problema exigem que:

$$
\mathbf{H}^{\prime}(\mathbf{x}) \mathbf{R}^{-1}[\mathbf{z}-\mathbf{h}(\mathbf{x})]-\rho \mathbf{C}^{\prime}(\mathbf{x}) \mathbf{c}(\mathbf{x})=\mathbf{0}
$$

que pode ser reescrita, pela introdução da variável $\boldsymbol{\lambda}$, como:

$$
\begin{array}{r}
\mathbf{H}^{\prime}(\mathbf{x}) \mathbf{R}^{-1}[\mathbf{z}-\mathbf{h}(\mathbf{x})]+\mathbf{C}^{\prime}(\mathbf{x}) \boldsymbol{\lambda}=\mathbf{0} \\
\mathbf{c}(\mathbf{x})+\frac{1}{\rho} \boldsymbol{\lambda}=\mathbf{0}
\end{array}
$$

Linearizando o sistema em torno do ponto $\mathbf{x}^{v}$, a solução ótima pode ser obtida iterativamente considerando o seguinte sistema de equações:

$$
\left[\begin{array}{rc}
\mathbf{G}\left(\mathbf{x}^{v}\right) & -\mathbf{C}^{\prime}\left(\mathbf{x}^{v}\right) \\
-\mathbf{C}\left(\mathbf{x}^{v}\right) & -1 / \rho
\end{array}\right]\left[\begin{array}{c}
\Delta \mathbf{x} \\
\boldsymbol{\lambda}^{v+1}
\end{array}\right]=\left[\begin{array}{c}
\mathbf{H}^{\prime}\left(\mathbf{x}^{v}\right) \mathbf{R}^{-1} \mathbf{r}\left(\mathbf{x}^{v}\right) \\
\mathbf{c}\left(\mathbf{x}^{v}\right)
\end{array}\right]
$$

Para valores muitos grandes de $\rho$, esta última equação resume-se, portanto, à Eq. (2.29).

\subsubsection{Modelo de medição linearizado}

Baseando-se nos princípios que norteiam o chamado Fluxo de Carga Linearizado (ou Fluxo $\mathrm{CC}$ ), é possível deduzir-se um modelo de EE simplificado onde somente o acoplamento $P-\theta$ é con- 
siderado. Neste contexto, as magnitudes das tensões nas barras tornam-se conhecidas, possuindo valor de 1 p.u, e os elementos shunt e resistências nos ramos são desprezados. Adicionalmente, assume-se que as aberturas angulares nos ramos são pequenas, e que é válida a aproximação $\operatorname{sen} \theta_{k m} \cong \theta_{k m}$.

Como consequência, uma medida de fluxo de potência entre as barras $k$ e $m$ poderá ser aproximada por:

$$
P_{k m}=\frac{\theta_{k}-\theta_{m}}{x_{k m}}+e_{p k m}
$$

em que $\theta_{k}$ e $\theta_{m}$ representam, respectivamente, os ângulos das tensões complexas nas barras $k$ e $m ; x_{k m}$ é a reatância série do ramo $k-m$; e $e_{p k m}$ denota o erro associado à medida. Uma medida de injeção de potência ativa em uma determinada barra $k$, representada pelo somatório dos fluxos nas linhas adjacentes, será dada por:

$$
P_{k}=\sum_{m \in K} P_{k m}+e_{p}
$$

onde $e_{p}$ simboliza o erro associado à medida.

O modelo de medição do Estimador de Estado Linearizado (EEL) pode ser estabelecido como segue:

$$
\begin{gathered}
\mathbf{z}_{\mathbf{p}}=\mathbf{H}_{\mathbf{p}} \boldsymbol{\theta}+\mathbf{e}_{\mathbf{p}} \\
E\left(\mathbf{e}_{\mathbf{p}}\right)=0, E\left(\mathbf{e}_{\mathbf{p}} \mathbf{e}_{\mathbf{p}}^{\prime}\right)=\mathbf{R}_{\mathbf{p}}
\end{gathered}
$$

onde $\mathbf{z}_{\mathbf{p}}, \mathbf{e}_{\mathbf{p}}$ e $\mathbf{R}_{\mathbf{p}}$ são as mesmas grandezas definidas no EEDR, que correspondem à parte ativa do modelo. Nesse caso, como as quantidades medidas estão linearmente relacionadas ao estado, a matriz $\mathbf{H}_{\mathbf{p}}$ (ou matriz de observação) será constante, em função unicamente das reatâncias do sistema. A solução do problema utilizando MQP poderá ser obtida em um único passo, da seguinte forma:

$$
\mathbf{G}_{\mathbf{p}} \hat{\boldsymbol{\theta}}=\mathbf{H}_{\mathbf{p}}^{\prime} \mathbf{R}_{\mathrm{p}}^{-1} \mathbf{z}_{\mathrm{p}}
$$

onde $\mathbf{G}_{\mathbf{p}}=\mathbf{H}_{\mathbf{p}}^{\prime} \mathbf{R}_{\mathbf{p}}^{-\mathbf{1}} \mathbf{H}_{\mathbf{p}}$ é a matriz Ganho e $\hat{\boldsymbol{\theta}}$ é a estimativa do estado.

O modelo EEL é muitas vezes utilizado para se verificar a viabilidade de estratégias de solução do problema de EE pela análise simplificada do plano de medição. Assume-se normalmente que medidas ativas e reativas são fornecidas aos pares, portanto, resultados obtidos para as primeiras podem ser diretamente estendidos para as últimas. 


\subsubsection{Detecção e Identificação de Erros Grosseiros}

Uma das principais características do processo de Estimação de Estado é a possibilidade de rejeição em tempo real das medidas que não se compatibilizam com o modelo do sistema. Uma pré-análise dos limites de certas variáveis ou valores esperados pode eliminar importantes fontes de erro. Todavia, inevitavelmente, medidas portadoras de desvios grosseiros passarão indetectadas nesta fase inicial, havendo a necessidade de serem identificadas e excluídas do processo.

É possível mostrar que, na ausência de erros grosseiros, o índice $J(x)$ é uma variável aleatória com distribuição $\left(\chi^{2}\right)$ (qui-quadrado) com $m-n$ graus de liberdade. A deteç̧ão de erros grosseiros poderá ser então efetuada aplicando-se um teste estatístico de hipóteses, comparando-se o valor do índice convergido com um parâmetro pré-determinado. Caso a hipótese seja aceita, considera-se que existem erros grosseiros no modelo, que devem ser localizados e eliminados.

Uma das formas mais utilizadas para se identificar medidas errôneas consiste na análise dos resíduos normalizados, que são calculados dividindo-se cada componente do vetor de resíduos (r) pelo desvio padrão correspondente obtido da matriz covariância dos resíduos (W), ou seja:

$$
r_{N, k}=\frac{r_{k}}{\sqrt{w_{k k}}}
$$

onde $r_{N, k}$ é o resíduo normalizado associado à medida $k$; e $w_{k k}$ é o elemento na posição $k-k$ da matriz covariância dos resíduos, definida como:

$$
\mathbf{W}=\mathbf{R}-\mathbf{H G}^{-1} \mathbf{H}^{\prime}
$$

É possível mostrar que, se apenas uma medida no plano de medição for portadora de erro grosseiro e as demais medidas perfeitas, o maior resíduo normalizado estará associado à medida com erro (CLEMENTS; DAVIS, 1986). Desde que o nível de redundância seja adequado, esta propriedade também será válida no caso de erros múltiplos. Várias metodologias para detecção de erros grosseiros foram desenvolvidas baseando-se neste princípio, onde ciclos sucessivos de deteç̧ão e reestimação são efetuados até que todas as medidas com erros sejam eliminadas. Nesse sentido, citam-se os trabalhos de Aboytes e Cory (1975), Garcia, Monticelli e Abreu (1979) e Monticelli e Garcia (1983), que aplicam o chamado Teste do Máximo Resíduo Normalizado, onde a presença de erros grosseiros estará associada a valores de resíduos normalizados acima de um dado limiar. 


\subsection{A Estimação de Estado Multiárea}

A Estimação de Estado Multiárea (EEMA) corresponde à aplicação de técnicas de EE a sistemas de potência interconectados ou com dimensões elevadas, onde a operação descentralizada apresenta vantagens consideráveis em relação à convencional. Os métodos de solução do problema de EEMA são normalmente classificados pelo tipo de arquitetura que operam, se hierárquica ou distribuída. Na Estimação de Estado Hierárquica (EEH) assume-se uma arquitetura do tipo estrela, onde partes das estimativas locais são transferidas para um centro de controle independente que estabelece a sincronização entre áreas e refina os estados de fronteira. Este esquema está ilustrado na Fig. (2.3). Os métodos hierárquicos são os modelos multiárea mais difundidos, porque em sua grande parte possuem formulações matemáticas simples e geralmente permitem o uso de técnicas tradicionais de EE e aplicações associadas.

Modelos com arquitetura distribuída, por outro lado, assumem que o sistema interconectado possui toda a infraestrutura operacional e de comunicação que permite o compartilhamento de dados entre áreas, especialmente aqueles relacionados à zonas de fronteira. Estes métodos normalmente não possuem a figura do elemento central supervisor, como ilustrado na Fig. (2.4), podendo nesse caso serem denominados de Descentralizados (GOMEZ-EXPOSITO et al., 2011). Há, porém, modelos que requerem uma área independente para troca de dados, como em Korres (2011). Embora a maioria dos métodos em EED obtenham as estimativas consideradas "ótimas" em EEMA, o fluxo de informações entre áreas exigido é, muitas vezes, impraticável.

No âmbito de EEMA, um SEP pode estar particionado de várias formas, obedecendo a limites geográficos, operacionais ou estruturais. Dentre as diversas configurações de áreas possíveis, podem ser citadas (VAN CUTSEM; RIBBENS-PAVELLA, 1983; GOMEZ-EXPOSITO et al., 2011):

- Áreas não sobrepostas: áreas que não possuem barras em comum, estando interligadas por ramos com terminação em barras de fronteira (ramos de interconexão), como exemplificado na Fig. (2.5a), na interligação entre as áreas 1 e 2;

- Áreas sobrepostas por barras de fronteira: nesse caso, sistemas adjacentes possuem barras de fronteira em comum, como mostrado na Fig. (2.5b). Esta topologia pode também ser estabelecida a partir de áreas não sobrepostas (Fig. 2.5a), adicionando-se barras fictícias nos pontos intermediários das linhas de interconexão, segundo a Fig. (2.5c).

- Áreas sobrepostas por linhas de interconexão: áreas vizinhas possuem um ramo de interconexão em comum, compartilhando todas suas barras terminais (Fig. 2.5d);

- Áreas sobrepostas estendidas: neste caso, sistemas adjacentes estão interligados por mais de um nível de barras de fronteira, como ilustrado na Fig. (2.5e). 


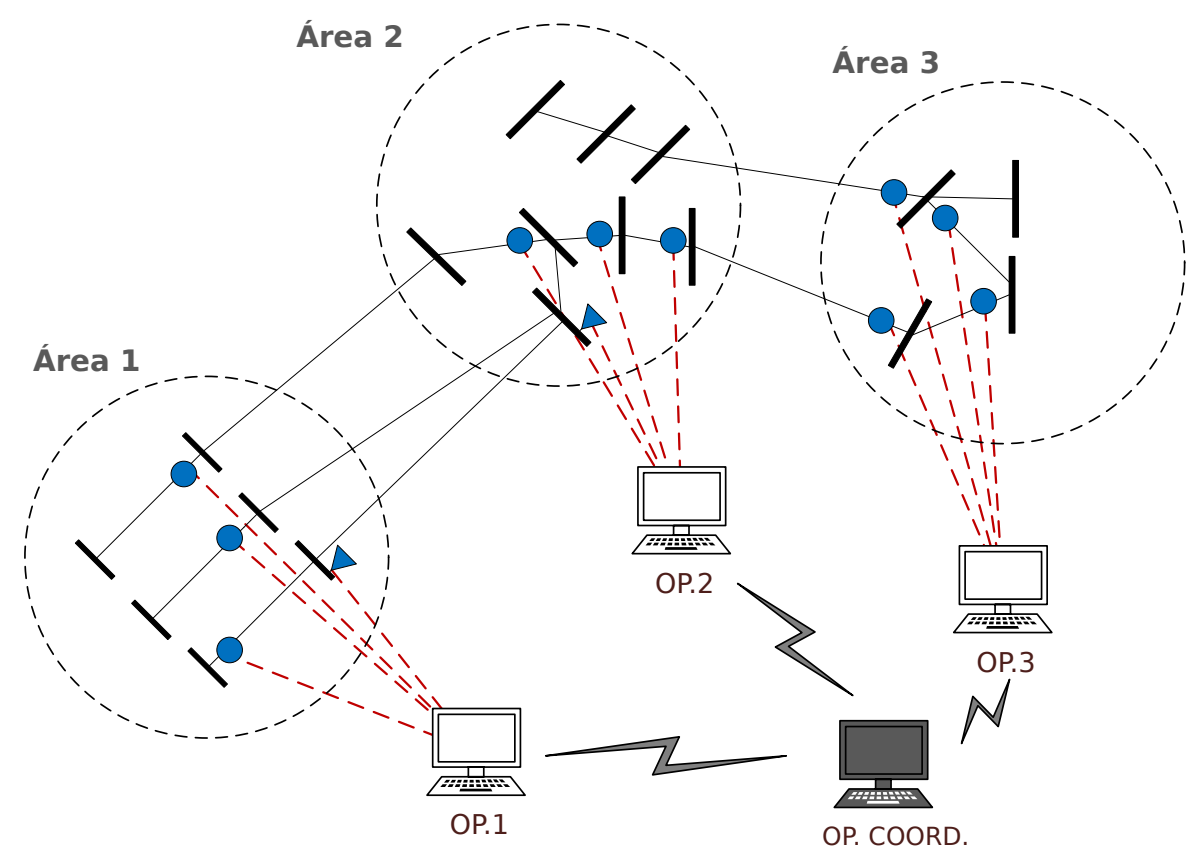

Figura 2.3 - Arquitetura de um Estimador de Estado Hierárquico

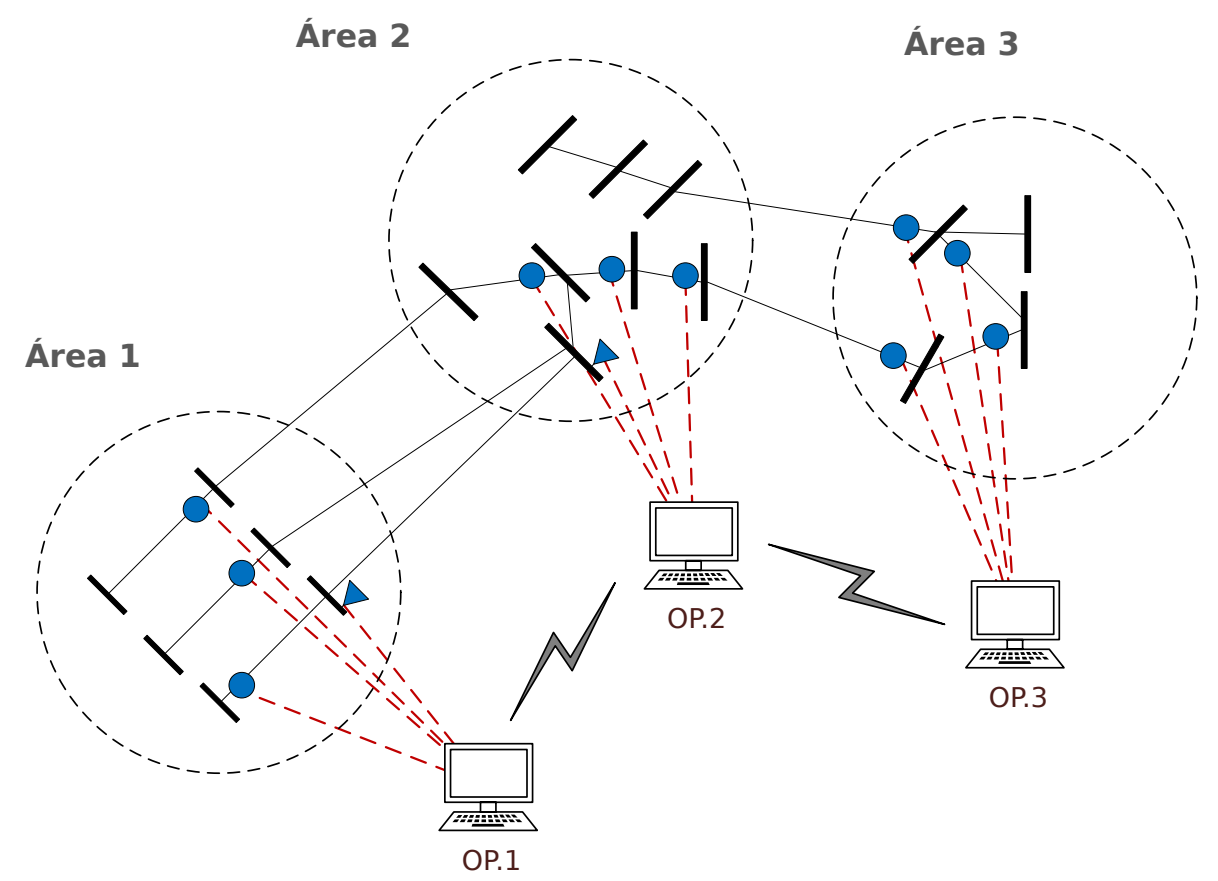

Figura 2.4 - Arquitetura de um Estimador de Estado Distribuído 


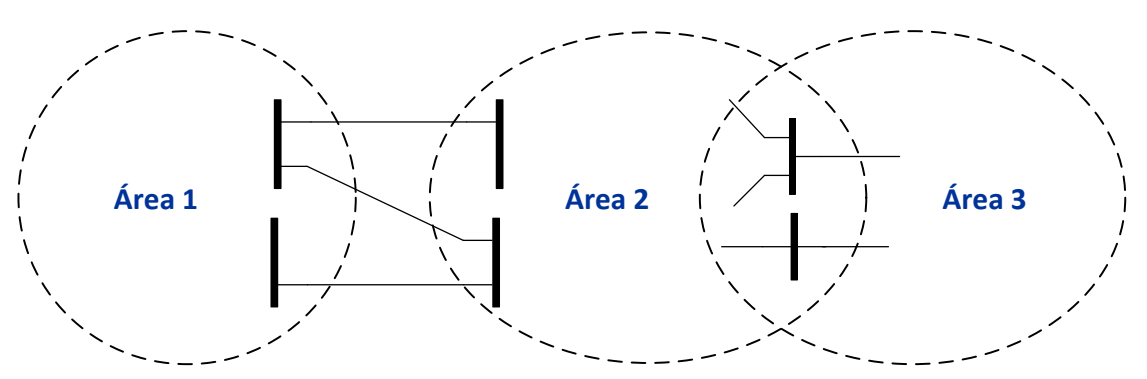

(a)

(b)

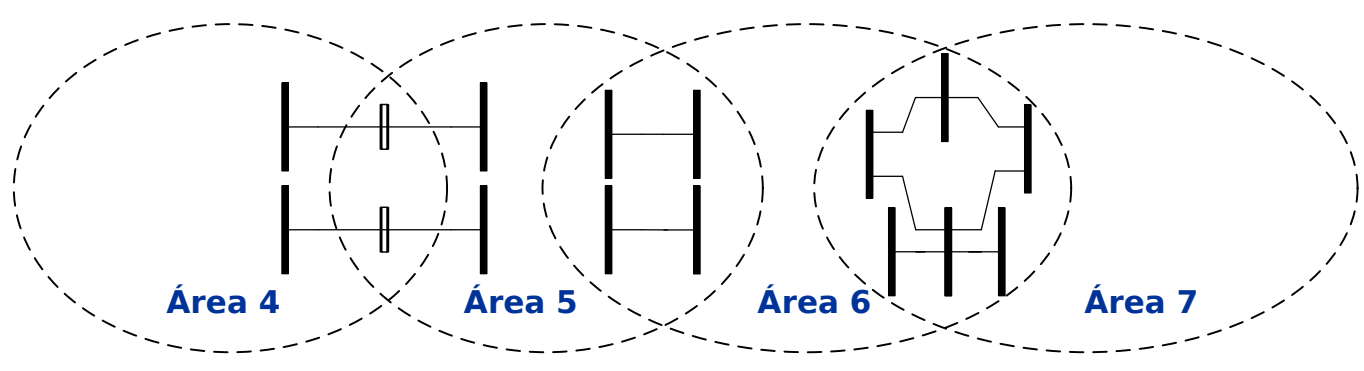

(c)

(d)

(e)

Figura 2.5 - Formas de decomposição existentes em ambientes descentralizados: (a) áreas não sobrepostas; (b) áreas sobrepostas por barras de fronteira; (c) áreas sobrepostas por barras fictícias em linhas de interconexão; (d) áreas sobrepostas por linhas de interconexão; (e) áreas sobrepostas estendidas.

De acordo com a estratégia de decomposição adotada, os seguintes tipos de barras estarão definidos:

- Barra interna: é uma barra local a uma determinada área e que não possui conexões com barras de áreas vizinhas;

- Barra de fronteira: barra local que está conectada a, no mínimo, uma barra pertencente a sistemas vizinhos;

- Barra de primeira vizinhança: barra que pertence a um sistema vizinho, mas que está conectada a uma barra de fronteira local. 


\subsubsection{Formulação do problema}

Um modelo de EE convencional quando aplicado a um SEP multiárea considerando todas as medidas existentes como pertencentes a um único sistema é geralmente denominado de modelo integrado ou completo. Resultados obtidos pelo Estimador de Estado Integrado (EEI) são considerados como ótimos no problema de EEMA (VAN CUTSEM; RIBBENS-PAVELLA, 1983; GOMEZ-EXPOSITO et al., 2011). Consequentemente, metodologias baseadas na minimização do índice $J(x)$ relacionado ao problema completo são também consideradas ótimas (CONEJO; TORRE; CANAS, 2007).

É possível constatar que a aplicação direta de modelos tradicionais de EE a sistemas multiárea é pouco viável, devido a uma série de fatores. Áreas adjacentes geralmente são relutantes em compartilhar dados entre si, especialmente informações da operação em tempo real. Adicionalmente, a sincronização de sistemas de supervisão distintos e a coordenação das estimativas locais dentro de margens de tempo satisfatórias se tornam muitas vezes impraticáveis. O elevado tráfego de dados exigido, a infraestrutura demandada e a implantação de medidas de segurança tornam-se também problemas difíceis de serem equacionados.

A motivação básica da EEMA está em se reduzir a complexidade associada ao problema completo, de elevada ordem, decompondo-o em subproblemas menores. Dentro do contexto de EE, em outras palavras, objetivam minimizar a função objetivo global com o auxílio de técnicas de descentralização.

Seja uma área arbitrária $a$ interligada a outras áreas por linhas de interconexão, como mostrado na Fig. (2.6). Considerando os tipos de barras definidos na Seção anterior, o vetor de estado da área $a\left(\mathbf{x}_{\mathbf{a}}\right)$ pode ser particionadas como segue:

$$
\mathbf{x}_{\mathbf{a}}=\left[\begin{array}{lll}
\mathbf{x}_{\mathbf{i} a}^{\prime} & \mathbf{x}_{\mathbf{b} a}^{\prime} & \mathbf{x}_{\mathbf{n} a}^{\prime}
\end{array}\right]^{\prime}
$$

em que:

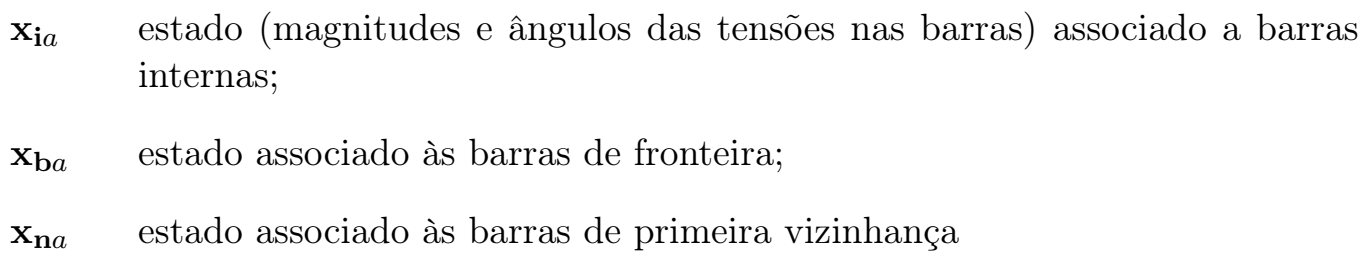

De forma similar, o vetor de medidas da mesma área estará definido como: 


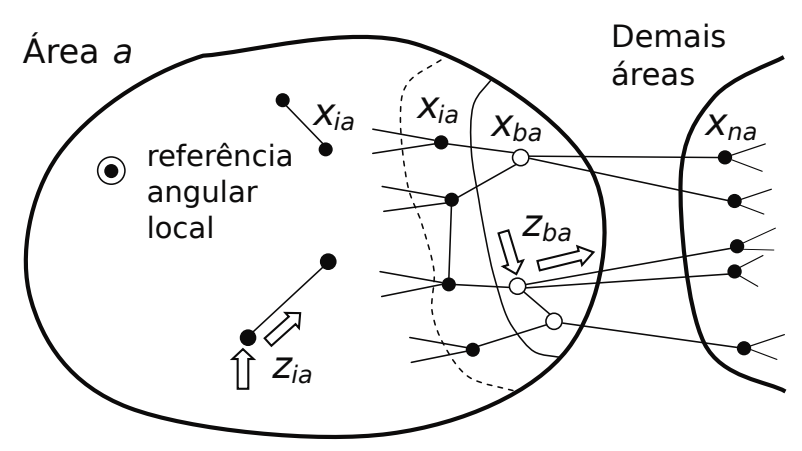

Figura 2.6 - Estimação de Estado Multiárea, definições e nomenclaturas ${ }^{2}$

$$
\mathbf{z}_{\mathbf{a}}=\left[\begin{array}{ll}
\mathbf{z}_{\mathbf{i} a}^{\prime} & \mathbf{z}_{\mathbf{b} a}^{\prime}
\end{array}\right]^{\prime}
$$

onde:

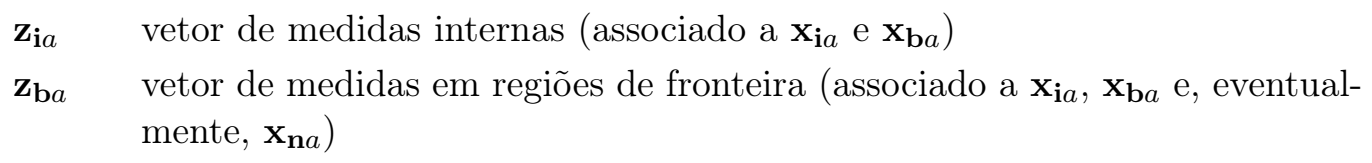

Suponha que o método dos Mínimos Quadrados Ponderados, na forma como estudado na Seção (2.1), seja escolhido para estimar o estado da área $a$. Nesse caso, o índice $J(x)$ correspondente será dado por:

$$
J\left(\hat{\mathbf{x}}_{\mathbf{a}}\right)=\frac{1}{2}\left[\mathbf{z}_{\mathbf{a}}-\mathbf{h}_{\mathbf{a}}\left(\hat{\mathbf{x}}_{\mathbf{a}}\right)\right]^{\prime} \mathbf{R}_{z a}^{-1}\left[\mathbf{z}_{\mathbf{a}}-\mathbf{h}_{\mathbf{a}}\left(\hat{\mathbf{x}}_{\mathbf{a}}\right)\right]
$$

Desprezando-se as medidas existentes em regiões de borda (fluxos em linhas de interconexões e injeções em barras de fronteira), cujas equações não lineares são dependentes do estado de sistemas vizinhos, o problema poderá ser resolvido da forma convencional, pelo método de Gauss-Newton (conforme Equações 2.4a e 2.4b). Ressalta-se que, neste cenário, estimativas de ângulo estarão baseadas na referência angular interna da área $a$.

Constata-se que, desde que seja possível processar estimadores independentes para cada área em um sistema interconectado, estimativas aceitáveis podem ser obtidas, com boas características

\footnotetext{
${ }^{2}$ Gráfico baseado no original em Gomez-Exposito et al. (2011), mas modificado de forma que $\mathbf{x}_{\mathbf{n} a}$ corresponderá a barras de primeira vizinhança externas, e não internas. Desta forma, $\mathbf{x}_{\mathbf{n} a}$ será equivalente à notação $x_{i}^{\text {ext }}$ definida em Zhao e Abur (2005).
} 
de convergência e desempenho computacional (FALCAO; WU; MURPHY, 1995). No entanto, caso medidas de fronteira não sejam devidamente tratadas, o estado das barras próximas será fortemente discrepante em relação às estimativas ótimas. Uma alternativa para minimizar este problema é aumentar a redundância nas regiões de interligação, mas ainda assim a sensibilidade entre áreas adjacentes permanece comprometida. Menciona-se ainda que, caso não haja uma estratégia eficiente para a sincronização das diferentes referências angulares, não será possível obter um panorama completo do sistema analisado, nem estabelecer, por exemplo, os fluxos interáreas.

\subsubsection{Estimadores Hierárquicos baseados em Duas Etapas}

As primeiras tentativas para resolver os problemas mencionados anteriormente levaram ao desenvolvimento dos métodos de EEMA baseado em duas etapas de estimação. A seguir são analisados dois dos modelos mais difundidos nesse contexto.

\section{O método STLSE}

Proposto por Van Cutsem, Horward e Ribbens-Pavella (1981), o método padrão de dois níveis, ou Standard Two-Level State Estimator (STLSE) considera um SEP cujas áreas estão associadas por meio de ramos de interconexão (linhas ou transformadores), como mostrado na Fig. (2.7).

A solução do problema de EEMA é obtida em dois níveis de estimação, da seguinte forma:

Nível 1 - Estimadores Individuais

A primeira etapa compreende o processamento de estimadores de estado convencionais, em cada uma das $n_{a}$ áreas existentes. Para um dado sistema $a$, o vetor de estado correspondente será dado por:

$$
\mathbf{y}_{\mathbf{a}}=\left[\begin{array}{ll}
\mathbf{y}_{\mathbf{i} a}^{\prime} & \mathbf{y}_{\mathbf{b} a}^{\prime}
\end{array}\right]^{\prime}
$$

onde a notação y indica que os ângulos das tensões nas barras estão sob a referência angular interna (local) da área. O vetor de medidas local será definido como:

$$
\mathbf{z}_{\mathbf{a}}=\left[\mathbf{z}_{\mathbf{i} a}\right]
$$

Nesse caso, medidas de injeção de potência em barras de fronteira e fluxos em linhas de interconexão, que estão relacionadas a variáveis de estado do sistema externo, são descartadas. 


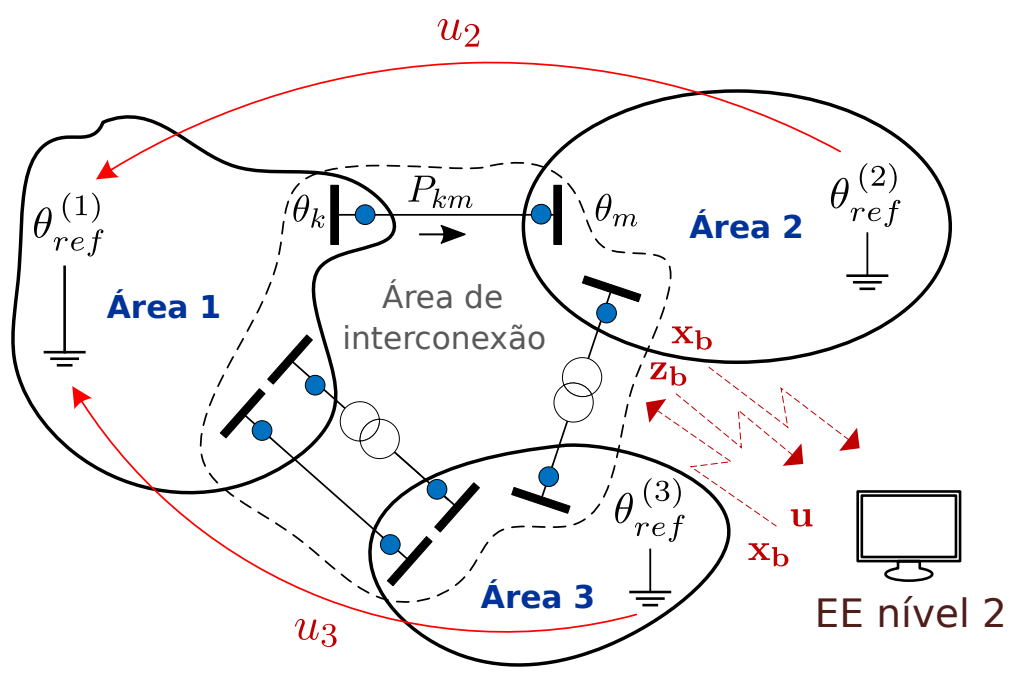

Medida de fluxo de potência

Figura 2.7 - Organização do Sistema no método STLSE (baseado no original em Van Cutsem, Horward e Ribbens-Pavella (1981).

O modelo de medição da área $a$ será, então, representado por:

$$
\mathbf{z}_{\mathbf{a}}=\mathbf{h}_{\mathbf{a}}\left(\mathbf{y}_{\mathbf{a}}\right)+\mathbf{e}_{\mathbf{z}_{a}}
$$

$\operatorname{com} E\left(\mathbf{e}_{\mathbf{z}_{a}}\right)=0, E\left(\mathbf{e}_{\mathbf{z}_{a}} \mathbf{e}_{\mathbf{z}_{a}}^{\prime}\right)=\mathbf{R}_{\mathbf{z}_{a}}$.

O problema representado pela Eq. (2.46) pode ser resolvido utilizando qualquer método convencional de EE, como já abordado em seções anteriores. Após a convergência do processo iterativo, o estado estimado $\hat{\mathbf{y}}_{\mathbf{a}}$ será obtido, juntamente com a matriz covariância do estado estimado $\left(\mathbf{R}_{\hat{\mathbf{y}}_{\mathbf{a}}}\right)$, definida como:

$$
\mathbf{R}_{\hat{\mathbf{y}}_{\mathbf{a}}}=\mathbf{G}_{\mathbf{a}}^{-1}
$$

onde $\mathbf{G}_{\mathbf{a}}$ é a matriz ganho local. A componente do vetor de estado relacionada às barras de fronteira $\left(\mathbf{y}_{\mathbf{b} a}\right)$ será, então, enviada ao centro de controle de coordenação, juntamente com a matriz $\mathbf{R}_{\hat{\mathbf{y}}_{\mathbf{a}}}$. 
Nível 2 - Estimador de Coordenação

O principal objetivo desta etapa adicional é incorporar ao problema medidas de fluxos em linhas de interconexão ignoradas na etapa anterior. Como tais informações estão associadas a estados sob referências angulares distintas, a adoção de uma variável de sincronismo é necessária.

Uma vez processados os estimadores das $n_{a}$ áreas, o vetor de estado da área central (índice c) será definido como:

$$
\mathbf{y}_{\mathbf{c}}=\left[\begin{array}{ll}
\mathbf{y}_{\mathbf{b}}^{\prime} & \mathbf{u}^{\prime}
\end{array}\right]^{\prime}
$$

em que $\mathbf{y}_{\mathbf{b}}=\left[\begin{array}{llll}\mathbf{y}_{\mathbf{b} 1}^{\prime} & \mathbf{y}_{\mathbf{b} 2}^{\prime} & \ldots & \mathbf{y}_{\mathbf{b} n_{a}}^{\prime}\end{array}\right]^{\prime}$ é o vetor composto pelos estados de fronteira das $n_{a}$ áreas do sistema, e u é o vetor de dimensão $\left(n_{a}-1\right)$ de sincronismo angular, que representa as defasagens entre as referências angulares dos $\left(n_{a}-1\right)$ subsistemas em relação a uma referência global arbitrariamente escolhida $(u=0)$.

A aplicação da variável de sincronismo $u$ no âmbito de EEMA pode ser compreendida considerando novamente o modelo da Fig. (2.7), onde uma medida de fluxo de potência ativa está alocada entre as barras $k$, que pertence à área 1, e $m$, pertencente à área 2. Para o cálculo de $P_{k m}$, é necessário que $\theta_{m}$ (com referência angular em $\theta_{r e f}^{(2)}$ ) seja corrigida para a referência angular da barra $k\left(\theta_{\text {ref }}^{(1)}\right)$.

A partir do gráfico, considerando $\theta_{\text {ref }}^{(1)}=\theta_{\text {ref }}^{(2)}=0^{\circ}$, a seguinte relação será válida:

$$
\theta_{m}^{(2)}=\theta_{m}^{(1)}+u_{2}
$$

em que $\theta_{m}^{(2)}$ denota o ângulo da barra $m$ com referência na área 2 , e $\theta_{m}^{(1)}$ é o mesmo ângulo referenciado na área 1 . Nesta situação, a defasagem angular $\theta_{k m}$ será dada por:

$$
\theta_{k m}=\theta_{k}^{(1)}-\theta_{m}^{(1)}=\theta_{k}^{(1)}-\left(\theta_{m}^{(2)}-u_{2}\right)=\theta_{k}^{(1)}-\theta_{m}^{(2)}+u_{2}
$$

Assim, uma medida $P_{k m}$, com $k$ referenciada em $\theta_{r e f}^{1}$ e $m$ em $\theta_{r e f}^{2}$, será processada da seguinte forma:

$$
P_{k m}=V_{k}^{2} g_{k m}-V_{k} V_{m}\left(g_{k m} \cos \left(\theta_{k m}+u_{2}\right)+b_{k m} \operatorname{sen}\left(\theta_{k m}+u_{2}\right)\right)
$$

Semelhantemente, uma medida $Q_{k m}$ será expressa por: 


$$
Q_{k m}=-V_{k}^{2}\left(b_{k m}+b_{k m}^{s h}\right)-V_{k} V_{m}\left(g_{k m} \operatorname{sen}\left(\theta_{k m}+u_{2}\right)-b_{k m} \cos \left(\theta_{k m}+u_{2}\right)\right)
$$

Para o aumento da redundância do estimador central, estimativas de fronteira enviadas pelas subáreas serão integradas ao plano de medição como pseudomedidas, ponderadas pelos correspondentes elementos diagonais da matriz $\mathbf{R}_{\hat{\mathbf{y}}}$ (Eq. 2.47).

Sob estas considerações, o modelo de medição do Estimador de Coordenação pode então ser estabelecido como:

$$
\mathbf{z}_{\mathbf{c}}=\mathbf{h}_{\mathbf{c}}\left(\mathbf{y}_{\mathbf{c}}\right)+\mathbf{e}_{\mathbf{z}_{c}}
$$

onde $\mathbf{z}_{\mathbf{c}}=\left[\begin{array}{ll}\mathbf{z}_{\mathbf{u}}^{\prime} & \hat{\mathbf{y}}_{\mathbf{b}}^{\prime}\end{array}\right]^{\prime}$, sendo o vetor $\mathbf{z}_{\mathbf{u}}$ formado por medidas de fluxos de potência ativa e reativa, e o vetor $\hat{\mathbf{y}}_{\mathbf{b}}$ composto pelos estados estimados nas regiões de fronteira, calculados pelos EE locais. Em notação matricial, o modelo pode ser reescrito como:

$$
\left[\begin{array}{c}
\mathbf{z}_{\mathbf{u}} \\
\hat{\mathbf{y}}_{\mathbf{b}}
\end{array}\right]=\left[\begin{array}{c}
\mathbf{h}_{\mathbf{u}}\left(\mathbf{y}_{\mathbf{b}}, \mathbf{u}\right) \\
\mathbf{y}_{\mathbf{b}}
\end{array}\right]+\left[\begin{array}{l}
\mathbf{e}_{\mathbf{z}_{u}} \\
\mathbf{e}_{\hat{\mathbf{y}}_{b}}
\end{array}\right]
$$

onde considera-se que:

$$
\begin{aligned}
& E\left(\mathbf{e}_{\mathbf{z}_{\mathbf{u}}}\right)=0 \quad, \quad E\left(\mathbf{e}_{\mathbf{z}_{u}} \mathbf{e}_{\mathbf{z}_{u}}^{\prime}\right)=\mathbf{R}_{\mathbf{z}_{u}} \\
& E\left(\mathbf{e}_{\hat{\mathbf{y}}_{b}}\right)=0 \quad, \quad E\left(\mathbf{e}_{\hat{\mathbf{y}}_{b}} \mathbf{e}_{\hat{\mathbf{y}}_{b}}^{\prime}\right)=\operatorname{diag}\left(\mathbf{R}_{\hat{\mathbf{y}}}\right)
\end{aligned}
$$

Para a minimização do índice quadrático

$$
J\left(\hat{\mathbf{y}}_{\mathbf{c}}\right)=\frac{1}{2}\left[\mathbf{z}_{\mathbf{c}}-\mathbf{h}_{\mathbf{c}}\left(\hat{\mathbf{y}}_{\mathbf{c}}\right)\right]^{\prime} \mathbf{R}_{z_{c}}^{-1}\left[\mathbf{z}_{\mathbf{c}}-\mathbf{h}_{\mathbf{c}}\left(\hat{\mathbf{y}}_{\mathbf{c}}\right)\right]
$$

tem-se que:

$$
\left.\frac{\partial J}{\partial \mathbf{y}}\right|_{\mathbf{y}=\hat{\mathbf{y}}_{c}}=\mathbf{0} \quad \Rightarrow \quad \mathbf{H}^{\prime}\left(\hat{\mathbf{y}}_{\mathbf{c}}\right) \mathbf{R}_{z_{c}}^{-1}\left[\mathbf{z}_{\mathbf{c}}-\mathbf{h}_{\mathbf{c}}\left(\hat{\mathbf{y}}_{\mathbf{c}}\right)\right]=\mathbf{0}
$$

onde $\mathbf{H}\left(\hat{\mathbf{y}}_{\mathbf{c}}\right)=\left.\frac{\partial \mathbf{h}}{\partial \mathbf{y}}\right|_{\mathbf{y}=\hat{\mathbf{y}}_{c}}$. Esta última matriz possuirá a seguinte estrutura: 


$$
\mathbf{H}\left(\mathbf{y}_{\mathbf{c}}\right)=\left[\begin{array}{ll}
\frac{\partial \mathbf{h}_{u}}{\partial \mathbf{y}_{\mathbf{b}}} & \frac{\partial \mathbf{h}_{u}}{\partial \mathbf{u}} \\
\frac{\partial \mathbf{y}_{\mathbf{b}}}{\partial \mathbf{y}_{\mathbf{b}}} & \frac{\partial \mathbf{y}_{\mathbf{b}}}{\partial \mathbf{u}}
\end{array}\right]=\left[\begin{array}{cc}
\mathbf{H}_{\mathbf{b}} & \mathbf{H}_{u} \\
\mathbf{I} & \mathbf{0}
\end{array}\right]
$$

Finalmente, a estimativa da etapa de coordenação pode ser obtida através da seguinte expressão:

$$
\hat{\mathbf{y}}_{\mathbf{c}}^{v+1}=\hat{\mathbf{y}}_{\mathbf{c}}^{v}+\mathbf{G}^{-1} \mathbf{H}^{\prime}\left(\hat{\mathbf{y}}_{\mathbf{c}}^{v}\right) \mathbf{R}_{z_{c}}^{-1}\left[\mathbf{z}_{\mathbf{c}}-\mathbf{h}_{\mathbf{c}}\left(\hat{\mathbf{y}}_{\mathbf{c}}^{v}\right)\right]
$$

onde $\mathbf{G}^{-1}=\mathbf{H}^{\prime}\left(\hat{\mathbf{y}}_{\mathbf{c}}^{v}\right) \mathbf{R}_{z_{c}}^{-1} \mathbf{H}\left(\hat{\mathbf{y}}_{\mathbf{c}}^{v}\right)$.

Encerrada esta etapa, as estimativas nas barras de fronteira $\left(\hat{\mathbf{y}}_{\mathbf{b}}\right)$ são reenviadas às respectivas áreas, juntamente com o vetor de sincronismo u. Para o subsistema a, o vetor de estado com referência global $\left(\hat{\mathbf{x}}_{\mathbf{a}}\right)$ poderá então ser determinado a partir do estado estimado local $\hat{\mathbf{y}}_{\mathbf{a}}$, da seguinte forma:

$$
\hat{\mathbf{x}}_{\mathbf{a}}=\hat{\mathbf{y}}_{\mathbf{a}}+u_{a}
$$

em que $u_{a}$ representa o deslocamento a ser somado às estimativas de ângulo locais para sincronizálas na referência global do sistema.

Apesar de não fornecer resultados ótimos, o modelo STLSE apresenta um desempenho eficiente, no sentido que é adaptável a sistemas com diversos tipos de decomposições, além de ser compatível com algoritmos clássicos de estimação de estado e requerer um reduzido tráfego de dados entre áreas. Não obstante, sua estratégia de tratamento de informações entre áreas é deficiente, ao assumir que linhas de interconexão obrigatoriamente devem possuir medidas de fluxo em ambas as terminações e que, caso haja medidas de injeção na fronteira, as mesmas não sejam medidas $\operatorname{críticas}^{3}$ (HABIBALLAH, 1996).

\footnotetext{
${ }^{3}$ Medida crítica é aquela que, quando removida do sistema, torna o mesmo não observável. O termo $k$-tupla crítica é uma generalização deste conceito, ou seja, um conjunto de $k$ medidas não críticas onde a remoção simultânea de todas elas torna o sistema não observável.
} 


\section{O método ZHABSE}

A proposta de Zhao e Abur (2005), referido neste texto por ZHABSE (Zhao-Abur Multiarea State Estimator), constitui uma extensão do método padrão de duas etapas, onde aplica-se o conceito de áreas sobrepostas por linhas de interconexão (Fig. 2.5d). Nesse caso, os limites de áreas são estendidos às barras de primeira vizinhança, tornando possível a incorporação de medidas de fronteira tanto na estimação local quanto na etapa de coordenação.

Partindo da formulação em duas etapas já discutida anteriormente, o modelo de medição local em uma área arbitrária a será definido, no método ZHABSE, como:

$$
\mathbf{z}_{\mathbf{a}}=\mathbf{h}_{\mathbf{a}}\left(\mathbf{y}_{\mathbf{a}}\right)+\mathbf{e}_{\mathbf{z}_{a}}
$$

em que:

$$
\begin{gathered}
\mathbf{y}_{\mathbf{a}}=\left[\begin{array}{lll}
\mathbf{y}_{\mathbf{i} a}^{\prime} & \mathbf{y}_{\mathbf{b} a}^{\prime} & \mathbf{y}_{\mathbf{n} a}^{\prime}
\end{array}\right]^{\prime} \\
\mathrm{e} \\
\mathbf{z}_{\mathbf{a}}=\left[\begin{array}{ll}
\mathbf{z}_{\mathbf{i} a}^{\prime} & \mathbf{z}_{\mathbf{b} a}^{\prime}
\end{array}\right]^{\prime} .
\end{gathered}
$$

Nota-se que a adição da componente $\mathbf{z}_{\mathbf{b} a}$ ao vetor de medidas, constituída por injeções de potência em barras de fronteira e fluxos em interconexões, demanda a inclusão da componente $\mathbf{y}_{\mathbf{n} a}$ no estado, relacionada a barras de primeira vizinhança. Como consequência, para uma mesma barra de fronteira, haverão duas estimativas, uma local e outra advinda da área adjacente.

Ao final da primeira etapa, os vetores de estado locais, referentes às barras de fronteira $\left(\hat{\mathbf{y}}_{\mathbf{b}}=\left[\begin{array}{llll}\hat{\mathbf{y}}_{\mathbf{b} 1}^{\prime} & \hat{\mathbf{y}}_{\mathbf{b} 2}^{\prime} & \ldots & \hat{\mathbf{y}}_{\mathbf{b} n_{a}}^{\prime}\end{array}\right]^{\prime}\right)$ e barras de primeira vizinhança $\left(\hat{\mathbf{y}}_{\mathbf{n}}=\left[\begin{array}{llll}\hat{\mathbf{y}}_{\mathbf{n} 1}^{\prime} & \hat{\mathbf{y}}_{\mathbf{n} 2}^{\prime} & \ldots & \hat{\mathbf{y}}_{\mathbf{n} n_{a}}^{\prime}\end{array}\right]^{\prime}\right)$ serão enviados à área central, juntamente com as respectivas matrizes covariâncias, para serem tratados como pseudomedidas. Informações sobre fluxos nas interconexões e injeções de potência em barras de fronteira também serão fornecidas. Ressalta-se que, para o tratamento destas últimas medidas, o envio da topologia dos ramos internos associados também será necessário.

Por fim, a etapa de coordenação é processada de forma simular ao STLSE, sob o seguinte modelo de medição:

$$
\mathbf{z}_{\mathbf{c}}=\mathbf{h}_{\mathbf{c}}\left(\mathbf{y}_{\mathbf{c}}\right)+\mathbf{e}_{\mathbf{z}_{\mathbf{c}}}
$$

onde $\mathbf{z}_{\mathbf{c}}=\left[\begin{array}{lll}\mathbf{z}_{\mathbf{u}}^{\prime} & \hat{\mathbf{y}}_{\mathbf{b}}^{\prime} & \hat{\mathbf{y}}_{\mathbf{n}}^{\prime}\end{array}\right]^{\prime}$. Nesse caso, estarão também incluídas no vetor $\mathbf{z}_{u}$ medidas de injeção de potência em barras de fronteira, que passarão a estar em função do novo vetor de estado $\mathbf{y}_{\mathbf{c}}=\left[\begin{array}{lll}\mathbf{y}_{\mathbf{b}}^{\prime} & \mathbf{y}_{\mathbf{n}}^{\prime} & \mathbf{u}^{\prime}\end{array}\right]^{\prime}$. 
O método ZHABSE apresenta desempenho computacional compatível ao STLSE, com a vantagem da inclusão de medidas não tratadas por este último. Não obstante, o modelo ainda sofre com algumas deficiências comuns aos métodos de duas etapas, como o problema de elevadas dimensões na área de coordenação (KURZYN, 1983).

\subsubsection{Histórico e Trabalhos Correlatos}

Nesta seção pretende-se discorrer sobre alguns dos trabalhos mais relevantes no contexto da EEMA, ressaltando suas principais características e contribuições. Estudos específicos sobre o estado da arte no tema, classificação das metodologias e questões computacionais podem também ser vistos em Van Cutsem e Ribbens-Pavella (1983) e Gomez-Exposito et al. (2011).

As primeiras menções sobre técnicas de EE para sistemas de grande porte surgiram já nos trabalhos fundamentais publicados (SCHWEPPE; WILDES, 1970; SCHWEPPE; ROM, 1970; SCHWEPPE, 1970). Em sua proposta, o pesquisador menciona alternativas para a redução do esforço computacional no método MQP explorando os conceitos de Quantização Espacial e Varredura Espacial. Em ambos os casos, assume-se que o sistema é formado por áreas sobrepostas separadas por barras fictícias que dividem as linhas de interconexão. Na primeira técnica, cada subárea é responsável por processar um estimador local com as medidas internas correspondentes, sem nenhuma etapa posterior de centralização. Na segunda estratégia, após a obtenção das estimativas individuais, os estados das barras de fronteira são reestimados considerando como pseudomedidas os estados estimados nas barras fictícias. Ambas as técnicas podem ser incluídas na categoria dos métodos de Estimação de Estado Distribuída (EED).

Posteriormente, outros trabalhos nesta mesma linha foram também desenvolvidos por Sasaki, Aoki e Yokoyama (1987) e Ebrahimian e Baldick (2000), dentre outros. Técnicas correlatas também são citadas em Gomez-Exposito et al. (2011). Os métodos distribuídos em geral consideram a existência de constantes links de comunicação entre as áreas. Tal premissa, contudo, é pouco condizente com o panorama estrutural atualmente presente nos SGEs modernos.

Seguindo a tendência da aplicação de estratégias de controle hierárquicas em SEPs, por volta da década de 70-80 iniciaram-se também os primeiros esforços no desenvolvimento de modelos hierárquicos para EEMA. Além do método STLSE, descrito na seção anterior, metodologias que envolvem outras formas de particionamento interárea também foram propostas, como por exemplo, nos trabalhos de Wallach, Handschin e Bongers (1981), onde são consideradas áreas sobrepostas por linhas de interconexão e em Seidu e Mukai (1985), onde a decomposição é realizada simplesmente pela configuração da matriz ganho. Van Cutsem e Ribbens-Pavella (1983) apresentam um comparativo entre diversos modelos de EEH, especialmente sob as óticas de robustez, fluxos de dados envolvidos e tempo computacional, que eram os requisitos mais exigidos nos trabalhos pioneiros na problemática. 
No algoritmo proposto por Kurzyn (1983), baseado no método STLSE, a tarefa de reestimação do estado em barras de fronteira é eliminada da etapa de coordenação. O vetor de sincronismo angular (u) é então determinado por meio de um processo sequencial efetuado em cada linha de interligação, resultando em expressivos ganhos computacionais. De forma similar, o trabalho de Lo et al. (1988a) fundamenta-se na premissa que estimativas em fronteiras obtidas localmente são muito precisas e não necessitam ser reestimadas. Para a determinação do vetor de coordenação, somente medidas de fluxo ativo são consideradas. Testes computacionais comparando este último modelo ao algoritmo de Kurzyn e ao método STLSE são apresentados em Lo et al. (1988b).

Habiballah (1996) discorre sobre alterações no modelo STLSE convencional para a superação de duas dificuldades: o tratamento de medidas de injeções de fronteira e a obrigatoriedade da existência de medidas de fluxos nas linhas de interconexão. Para a primeira situação, o autor sugere a transformação de medidas de injeção em somatórios de fluxos internos, através da subtração dos fluxos estimados nas linhas adjacentes. As medidas resultantes são então tomadas como pseudomedidas na etapa posterior de centralização. Para o segundo problema, propõem-se também adaptações de medidas de forma a aumentar a redundância nas regiões de fronteira.

Três grandes questões associadas aos estimadores de dois níveis são tratadas por Lakshminarasimhan e Girgis (2007): a possibilidade de assincronismo, atraso, ou falha no envio de dados para a etapa de coordenação. A metodologia consiste, basicamente, em se aguardar os resultados dos diferentes estimadores locais para se efetuar a centralização. Caso o estado de qualquer um dos estimadores locais não esteja disponível, estimativas históricas são então utilizadas, ponderadas a partir de um determinado índice. No decorrer do processo iterativo, se o índice reduzir-se abaixo de um determinado limiar, o processamento do estado para a área com falta de dados será totalmente ignorado.

Em outro modelo de solução, Falcao, Wu e Murphy (1995) aplicam técnicas de otimização ao problema de estimação multiárea. Inicialmente, áreas adjacentes são consideradas sobrepostas por barras de fronteiras. Assume-se também que não há medidas de injeção em barras de fronteira, possibilitando então que injeções nulas entre áreas sobrepostas sejam tomadas como restrições de igualdade no modelo. Aplicando o princípio do desacoplamento do modelo e desprezando-se os elementos fora das diagonais existentes nas matrizes ganho, o problema é resolvido de forma distribuída, sendo que os estados associados às fronteiras são atualizados nas etapas locais.

De maneira equivalente, em Zhao, Guo e Fan (2008) são aplicadas estratégias de otimização sob a restrição que fluxos ativos e reativos associados a duas áreas vizinhas devem ser iguais. Desta forma, a sincronização é efetuada diretamente a partir do estado estimado e dos fluxos determinados na primeira etapa. Trabalhos nesta mesma linha podem ser vistos em Conejo, Torre e Canas (2007) e em Caro, Conejo e Minguez (2011), que utilizam técnicas de decomposição lagrangeana para estimar o estado global. 
Patel e Girgis (2007) propõem uma ligeira modificação no modelo de duas etapas proposto por Zhao e Abur, revisto na seção anterior, para permitir o tratamento de medidas de injeção de fronteira sem a necessidade do envio de informações topológicas dos ramos associados. Para tal, os autores sugerem a correção das medidas de injeção considerando os fluxos estimados nas linhas dos sistemas internos, de forma que as medidas só estarão relacionadas à fronteira e poderão ser usadas sem maiores dificuldades na etapa de coordenação.

Já Jiang, Vittal e Heydt (2007) ponderam que o impacto das medidas de uma determinada área no estado do subsistema vizinho será tanto mais abrangente quanto mais interconexões existirem, influenciando não somente barras de fronteira, mas também certas barras internas da área vizinha. Os autores propõem então um modelo multiárea baseado em dois níveis de estimação, onde barras internas que apresentarem elevada sensibilidade em relação às barras de fronteira terão seus estados reestimados. Na primeira etapa ocorre a estimação dos estados locais, de forma semelhante ao método STLSE, sendo que ao final do processo serão calculados, para cada barra, os índices de sensibilidade de fronteira. No segundo nível são tratadas medidas de fluxos interáreas e de injeções nas barras de fronteira, sendo incluídos também, como pseudomedidas, os estados estimados das barras com índice de sensibilidade superior a uma determinada margem. A sincronização das estimativas de ângulo é direta, na medida em que considera-se a disponibilidade de PMUs em todas as barras de referência do sistema.

Os mesmos autores do trabalho anterior propõem também em Jiang, Vittal e Heydt (2008) uma metodologia de EED com comportamento ótimo, baseada na teoria diacóptica, seguindo outros trabalhos da mesma linha (IRVING; STERLING, 1979; AHMED; BRAMELLER, 1991). Utilizando os princípios desta teoria, o problema de EE poderá ser resolvido de forma plenamente distribuída, mediante a remoção dos elementos situados fora da diagonal da matriz ganho e decomposição desta matriz em duas submatrizes, uma bloco-diagonal e outra auxiliar, não diagonal, de dimensão reduzida. A utilização de PMUs é fundamental para garantir a inversa da matriz ganho resultante. O problema de EE decomposto é resolvido a partir da interação entre subsistemas locais e um elemento intermediário independente, que efetua o tratamento de medidas interáreas e reenvia a cada área os estados de fronteira atualizados.

Korres (2011) também apresenta uma extensa metodologia no campo de EED, com convergência para o estado ótimo. A formulação matemática, baseada no MQP com restrições e otimização lagrangiana, toma como função objetivo a minimização mútua dos índices $J(x)$ locais e de um operador central. O problema é resolvido de forma descentralizada, em um processo iterativo com troca de dados entre os subsistemas e o elemento coordenador. Estratégias para análise de observabilidade e processamento de erros grosseiros também são propostas. A análise de desempenho do modelo foca-se principalmente no tempo computacional e no intercambio de dados em relação ao modelo integrado. 


\subsubsection{Perspectivas e Demandas Atuais}

Alguns dos principais desafios associados ao desenvolvimento de metodologias para EEMA são listados a seguir:

- Sincronismo: refere-se à necessidade de que medidas processadas em regiões geográficas distintas devem ser tomadas em instantes de tempo os mais próximos possíveis. O uso de PMUs destaca-se como uma das soluções mais atrativas para esta questão;

- Robustez: diz respeito à capacidade de convergência de um modelo de EEMA sob diferentes tipos de arquitetura, formas de decomposição e na presença de erros grosseiros, especialmente em regiões de fronteira;

- Intercâmbio de informações: premissa relativa à quantidade de dados transferidos no processo de estimação, entre áreas adjacentes, no caso de EED, ou entre áreas e um operador central, no caso de EEH. Problemas correlatos são o atraso na chegada de informações, a perda parcial de dados ou a falha total de algum subsistema;

- Precisão: refere-se à convergência para resultados ótimos, ou seja, para as mesmas estimativas do modelo integrado. O custo para a otimalidade, entretanto, é o aumento do fluxo de informações entre áreas e o uso de algoritmos não tradicionais para as funções de EE (VAN CUTSEM; RIBBENS-PAVELLA, 1983);

- Eficiência computacional: principal atrativo da EEMA, onde busca-se a redução da dimensionalidade do problema completo e do esforço computacional requerido;

- Compatibilidade: premissa indiretamente difundida, desejável em aplicações práticas que o modelo desenvolvido seja compatível com rotinas tradicionais já existentes nos centros de controle locais.

Stovall et al. (2006) estudam requisitos para a aplicação de modelos multiárea na dinâmica moderna dos SEPs, caracterizada pela presença de agentes como operadores de transmissão regionais e operadores independentes, em ambientes com estruturas cada vez mais desverticalizadas. Os autores sustentam que funções de análise e monitoramento dentro desse contexto são incompatíveis com modelos que assumem a centralização da operação. O trabalho disserta também sobre os requisitos de infraestrutura para uma manipulação eficiente dos dados envolvidos no processo, enfatizando que arquiteturas distribuídas e descentralizadas são mais adequadas ao panorama moderno. Ressalta-se ainda a importância de uma correta representação de redes externas em EEMA, reiterando-se também o papel das PMUs no problema como um todo. 


\section{Capítulo 3}

\section{Unidades de Medição Fasorial e Aplicações a Estimação de Estado}

Uma dos princípios que norteou o desenvolvimento das Unidades de Medição Fasorial, ou PMUs (do inglês, Phasor Measurement Units), surgiu com o chamado relé de distância de componentes simétricas, utilizado na proteção de SEPs. Este equipamento, aplicado para a monitoração de linhas de transmissão de alta tensão, foi um dos primeiros a possuir rotinas internas para o cálculo de componentes simétricas de tensão e corrente na frequência fundamental (PHADKE, 1993).

A evolução dos sistemas de comunicação, que permitiu que sinais separados por longas distâncias pudessem ser sincronizados de forma mais precisa, representou outra importante contribuição. Várias técnicas haviam sido inicialmente propostas para esta tarefa, como o uso de ondas de rádio portadoras de sinais de tempo padrões. Posteriormente, a implantação do conjunto de satélites relacionados ao Sinal de Posicionamento Global, do original inglês, Global Positioning System (GPS), veio a se estabelecer como solução padrão para esse problema.

As duas soluções mencionadas foram as principais responsáveis por viabilizar a tecnologia envolvida na construção de PMUs. Atualmente, estes dispositivos vêm se tornando cada vez mais indispensáveis na operação dos SGE modernos. Como muitos fenômenos elétricos são associados a diferenças angulares entre fasores, PMUs podem ser diretamente utilizados em funções de monitoramento, proteção e controle, provendo informações únicas, de forma rápida e precisa. O potencial de aplicação destes equipamentos a SEPs é ainda objeto de profunda investigação (BOSE, 2012).

Este capítulo pretende investigar os conceitos que fundamentam as PMUs, bem como as definições e normas relacionadas. Objetiva-se dar um enfoque particular nas aplicações destes dispositivos ao problema de Estimação de Estado, discorrendo sobre as formas de integração e os benefícios do uso de medidas fasoriais sincronizadas no modelo básico de EE e em EEMA. 


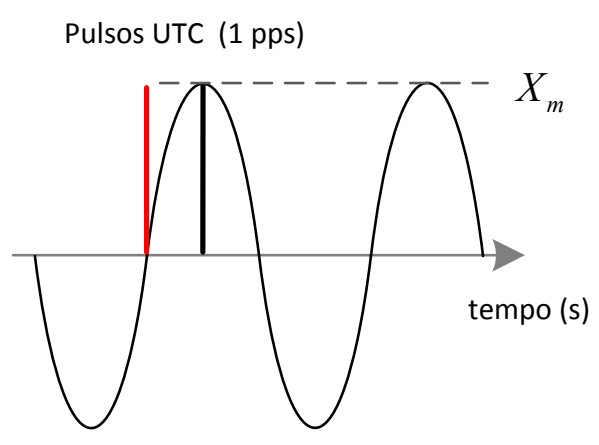

Sinal de entrada

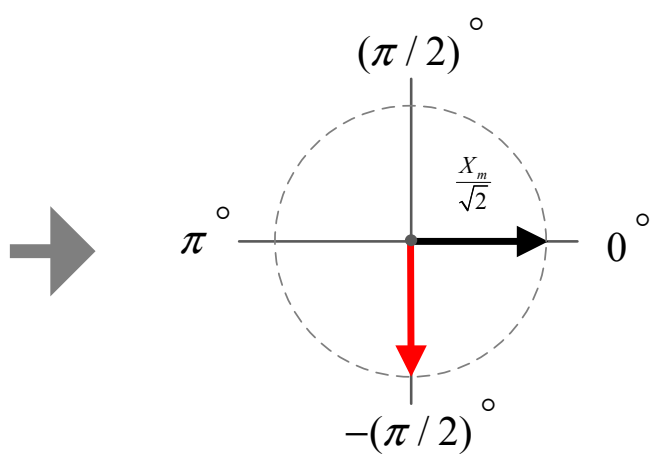

Sincrofasor

Figura 3.1 - Convenção para representação de sincrofasores

\subsection{Definições}

O termo fasor sincronizado ou sincrofasor foi padronizado pela primeira vez na norma IEEE 1344-1995, posteriormente atualizada e reeditada como IEEE Std C37.118-2005 (2005). Revisado em 2011, o padrão foi segmentado nas normas IEEE Std C37.118.1-2011 (2011), que trata do uso de medidas fasoriais sincronizadas em SEPs, métodos para quantificação e requisitos de desempenho, e na norma IEEE Std C37.118.2-2011 (2011), que define protocolos de comunicação e formatos para intercâmbio de dados.

Um sincrofasor possui conceito semelhante ao de um tradicional fasor utilizado para a representação de sinais de corrente alternada, com a diferença que seus valores são relativos a um sinal de tempo de referência. Especificamente, o sincrofasor $X$ de um sinal $x(t)$ é definido como sendo o seguinte número complexo:

$$
X=X_{r}+j X_{i}=\left(\frac{X_{m}}{\sqrt{2}}\right) e^{j \varphi}=\left(\frac{X_{m}}{\sqrt{2}}\right)(\cos \varphi+j \operatorname{sen} \varphi)
$$

em que $X_{m} / \sqrt{2}$ é o valor $R M S$ do sinal $x(t)$ e $\varphi$ é o ângulo instantâneo de $x(t)$ relativo a um sinal cossenoidal de referência, que possui frequência nominal e está sincronizado ao Tempo Universal Coordenado ou UTC (Universal Time Coordinated). Segundo esta definição, o ângulo $\varphi$ possui valor $0^{\circ}$ quando o máximo do sinal $x(t)$ ocorre imediatamente sobre os pulsos coordenados, $\mathrm{e}$ $-90^{\circ}$ caso os pulsos ocorram no valor zero (crescente), como mostrado na Fig. (3.1).

Ao contrário dos fasores convencionais, que são baseados em uma frequência característica implícita, sincrofasores permitem a representação de sinais com frequências distintas da nominal. 


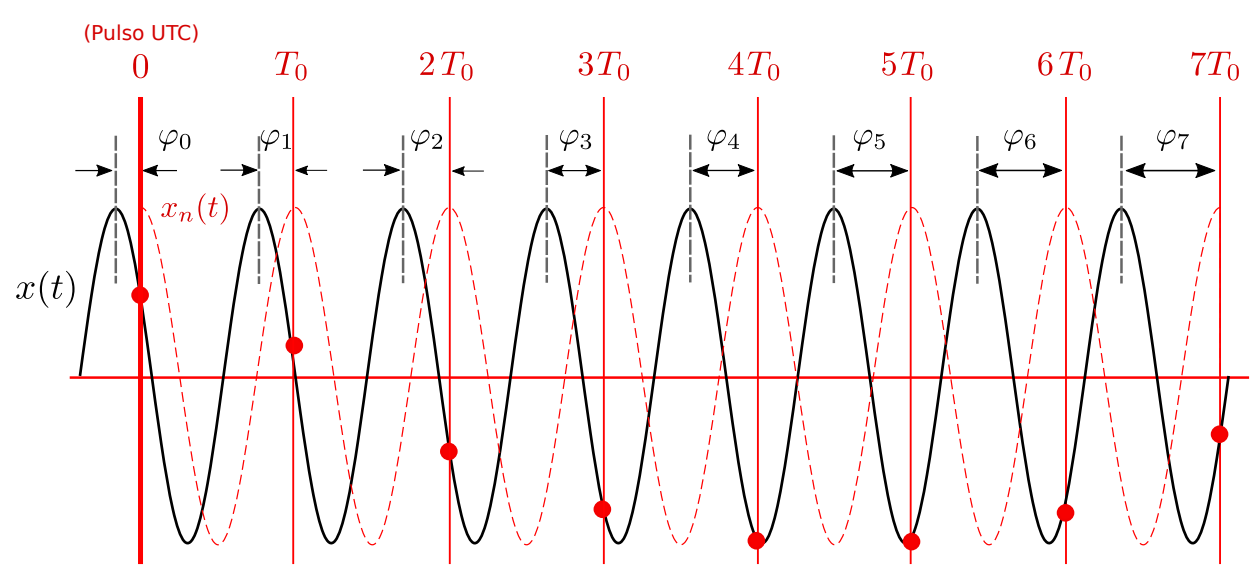

Figura 3.2 - Geração de sincrofasores para um sinal $x(t)$ com frequência distinta da nominal (adaptado de IEEE STD C37.118.1-2011).

No gráfico da Fig. (3.2) ilustra-se este fato, onde um sinal senoidal $x(t)$ com frequência $f=1 / T$ é observado nos intervalos $\left\{0, T_{0}, 2 T_{0}, \ldots, 7 T_{0}\right\}$, em que $f_{0}=1 / T_{0}$ é a frequência nominal do sistema. Na linha tracejada mostra-se a função cossenoidal de referência para o cálculo de sincrofasores $\left(x_{n}(t)\right)$, de período $T_{0}$, onde o marco inicial (0) coincide com o sinal UTC fornecido à taxa de 1-pps (pulso por segundo). Assumindo que $f \neq f_{0}$ e $f<2 f_{0}$, os fasores observados $\left\{X_{0}, X_{1}, X_{2}, \ldots, X_{7}\right\}$ possuirão magnitude constante e ângulos de fase variando uniformemente à taxa de $2 \pi\left(f-f_{0}\right) T_{0}$, conforme ilustrado na Figura. Considerando que $f_{0}=60 \mathrm{~Hz}$, os fasores serão reportados nesse caso à taxa de 60 frames $^{1}$ por segundo.

Uma Unidade de Medição Fasorial é um equipamento de medição (ou transdutor) que converte sinais analógicos trifásicos de tensão ou corrente em sincrofasores, sendo também capaz de determinar tanto a frequência do sistema quanto sua taxa de variação. Na Fig. (3.3) são mostrados os blocos básicos que integram uma PMU, que pode ser tanto um equipamento dedicado quanto uma aplicação secundária contida em outro dispositivo. O receptor de GPS produz um sinal de um pulso por segundo e uma etiqueta de tempo, consistindo em ano, dia, hora, minuto e segundo, associada ao tempo UTC. O sinal de 1-pps é segmentado em um oscilador interno para proceder à amostragem dos sinais analógicos (normalmente doze vezes por ciclo da frequência fundamental). Sinais de entrada são provenientes dos terminais secundários de Transformadores de Corrente (TCs) e Transformadores de Potencial (TPs), aos quais são aplicados filtros como o anti-aliasing, surge filtering e outros. O microprocessador determina, então, os sincrofasores

\footnotetext{
${ }^{1}$ Um frame representa um conjunto de medidas (sincrofasores, frequência, taxa de variação da frequência) relacionadas a um mesmo instante de tempo UTC. A norma IEEE Std C37.118.1-2011 (2011) estabelece que o número de frames por segundo (fps) deve ser submúltiplo da frequência nominal do sistema.
} 


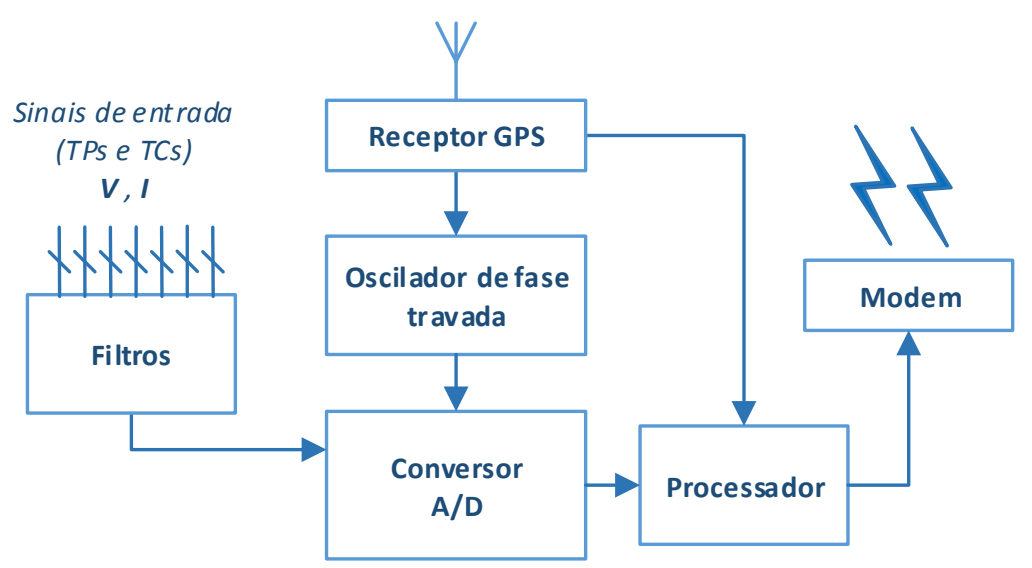

Figura 3.3 - Blocos funcionais de uma PMU (PHADKE; THORP, 2008)

correspondentes aos sinais de entrada, atribuindo a cada um deles etiquetas de tempo UTC. Por fim, os sinais são codificados e enviados pelos canais de comunicação aos concentradores de dados associados, ou Phasor Data Concentrators (PDCs), responsáveis por processar dados oriundos de várias unidades, organizando-os por instantes de tempo comuns e disponibilizando-os a outros PDCs ou componentes do sistema.

A integração de PMUs, PDCs, dispositivos de armazenamento de dados e sistemas de comunicação, com fins à aquisição e monitoramento de medidas fasoriais sincronizadas origina os chamados Sistemas de Medição Fasorial Sincronizada (SMFS). Dentre as aplicações dos SMFS em SEPs, podem ser citadas: controle em grandes áreas, monitoramento de oscilações e da frequência do sistema, análise de estabilidade, análise de contingências e modelagem de SEPs em tempo real. A principal vantagem desta tecnologia sobre o sistema SCADA tradicional é a possibilidade da aquisição e sincronização de medidas localizadas em áreas distantes geograficamente com elevada precisão e altas taxas de amostragem.

\subsubsection{Medidas disponibilizadas e precisão}

Uma vez alocada a uma barra, uma unidade de medição fasorial é capaz de prover o sincrofasor de tensão correspondente e os sincrofasores de corrente nas linhas adjacentes, como ilustrado na Fig. (3.4). As estimativas podem ser componentes monofásicas, de sequência positiva, ou ambas. O número efetivo de medidas disponibilizadas irá depender da quantidade de canais analógicos de entrada. Os medidores podem pertencer à classe de performance do tipo $P$ - apropriada para aplicações que demandam uma rápida resposta do equipamento, como em proteção de SEPs - ou à classe $M$, recomendada para as demais aplicações. 


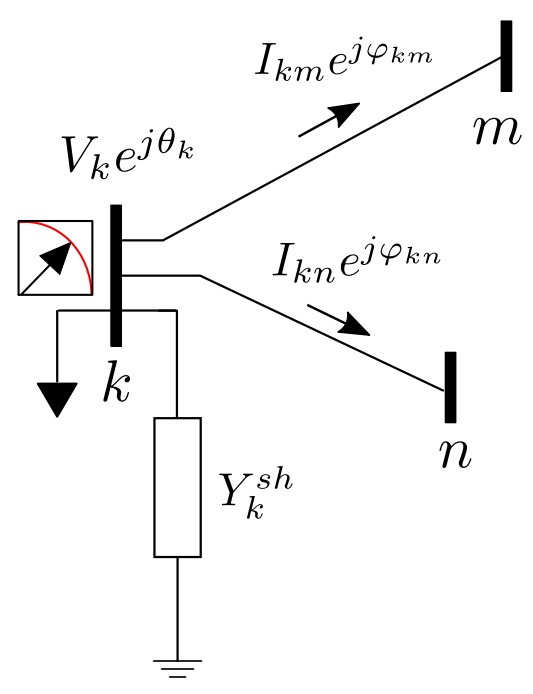

Figura 3.4 - Medidas disponibilizadas por uma PMU: sincrofasor de tensão em uma barra $k$ e sincrofasores de corrente em ramos adjacentes.

A precisão associada a sincrofasores é analisada em termos da métrica TVE, ou Total Erro do Vetor, computada pela diferença entre os sinais medidos e um sinal de referência, definido da seguinte forma:

$$
\operatorname{TVE}(n)=\sqrt{\frac{\left(\hat{X}_{r}(n)-X_{r}(n)\right)^{2}+\left(\hat{X}_{i}(n)-X_{i}(n)\right)^{2}}{\left(X_{r}(n)\right)^{2}+\left(X_{i}(n)\right)^{2}}}
$$

em que $\hat{X}_{r}(n)$ e $\hat{X}_{i}(n)$ são as partes real e imaginária do sinal medido no instante de tempo $n$, e $X_{r}(n)$ e $X_{r}(n)$ são os valores teóricos para o sinal de entrada no mesmo instante. A métrica TVE consegue captar três fontes de possíveis erros: magnitude, ângulo e assincronismo. Os valores limites para cada um destes parâmetros encontram-se definidos na norma, que considera também tolerâncias permissíveis de no máximo $1 \%$.

A precisão dos pulsos de GPS utilizados por PMUs é da ordem de 250 nanossegundos, fato que permite a sincronização de sinais a taxas menores que 1 microssegundo (ZHOU, 2008). Este valor, em um sistema de $60 \mathrm{~Hz}$, se traduz em medidas de ângulos com erros menores que $0,02^{\circ}$. Contudo, não obstante as rígidas margens estabelecidas pela norma, PMUs estão sujeitos a uma série de erros associados a TCs e TPs e outros equipamentos utilizados na cadeia de aquisição dos sinais de entrada, que podem deteriorar grandemente a qualidade das medidas geradas. Para a mitigação deste problema, técnicas apropriadas de calibração devem ser aplicadas, de forma que os desvios sejam compensados e que a precisão final não seja comprometida. 


\subsection{Sincrofasores no contexto de Estimação de Estado}

Muito antes da maturação da tecnologia de PMUs, pesquisadores já estudavam a influência de medidas de ângulo de tensão em estimação de estado. Thorp, Phadke e Karimi (1985) apresentam um estudo sobre o impacto dessas medidas na qualidade das estimativas finais no processo de EE. Ao efetuarem comparações entre medidas de ângulo e medidas de fluxo de potência, chegam à conclusão que os efeitos das primeiras só serão perceptíveis caso elas forem muito precisas. Afirmam também que um estimador com elevada proporção de medidas de ângulo em relação a medidas de injeção e fluxos de potência terá sua capacidade de rejeição de erros reduzida. Outros trabalhos que discutem sobre os benefícios do uso de medidas de ângulo podem ser vistos em Phadke, Thorp e Karimi (1986) e Phadke (1993).

No caso das medidas de ângulo provenientes de PMUs, a aplicação ao problema não é direta. Ocorre que em EE, à semelhança do problema de Fluxo de Carga, uma das barras é arbitrariamente escolhida como referência angular do sistema, sendo normalmente associado a ela o valor $0^{\circ}$. Já em PMUs, a referência dos fasores obtidos é determinada com base nos pulsos sincronizados pelo sinal de GPS, que é distinta da referência angular do sistema.

Zivanovic e Cairns (1996) sugerem a consideração das próprias medidas de ângulo fornecidas por PMUs como referências angulares. Um dos problemas, neste caso, é que as referências se tornarão medidas críticas, caso não haja uma redundância adequada. Os autores propõem, de forma alternativa, o uso de medidas de diferenças entre ângulos $\left(\theta_{i j}\right)$. Nesse caso, não haverá conflito, já que a medida usada será independente de referência angular. Zivanovic e Cairns também sugerem o uso de um modelo de EE regido somente por equações lineares em áreas cobertas por sincrofasores.

Nesse mesmo contexto, Zhu e Abur (2007) assumem a existência de no mínimo uma medida de ângulo no problema de EE, que será considerada a própria referência angular do sistema. Na falta desta, uma barra arbitrária é assumida como barra de referência, reduzindo o processo de estimação à forma convencional. No mesmo trabalho, os autores analisam questões como observabilidade e estratégias de identificação de erros grosseiros no novo paradigma proposto.

\subsubsection{Representação das medidas no modelo}

O modelo de medição original, estabelecido por Schweppe, busca a estimação do estado do sistema, através de medidas que, em sua maioria, estão relacionadas apenas indiretamente ao estado. Com a integração de medidas de PMUs, surgiu-se a possibilidade de medir diretamente o estado, ao invés de estimá-lo. Em termos de EE, o modelo de medição convencional, não linear, resume-se à sua forma linear, não iterativa, envolvendo cálculos matriciais de baixa complexidade. 
Esta premissa será, verdadeira, a priori, se PMUs forem instalados em todas as barras de uma determinada rede elétrica, fornecendo sincrofasores de ângulo e magnitude de tensão. Baldwin et al. (1993) mostram ainda que apenas $1 / 5$ a 1/4 de nós monitorados será suficiente para garantir a plena observabilidade. Mesmo assim, alguns fatores impedem a viabilização desta proposta a curto prazo, como a carência de sistemas de comunicação robustos que atendam ao elevado tráfego de informações processadas e fortes restrições relacionadas ao armazenamento e tratamento de dados (LIN et al., 2012).

Diante destes fatores, a solução considerada mais viável é a inclusão de medidas fasoriais em uma etapa de estimação subsequente, onde o modelo poderá ser resolvido de forma linear mantendo-se inalterada a infraestrutura do sistema SCADA. Zhao (2006) propõe dois modelos nesse contexto: o primeiro combina estimativas geradas por um EE não linear convencional a um estimador que considera medidas fasoriais e variáveis de estado na forma retangular; o segundo modelo também trabalha com uma etapa adicional linear, considerando sincrofasores de tensão na forma polar e sincrofasores de corrente convertidos em pseudomedidas de tensão. Uma estratégia muito similar a este segundo método pode ser vista em Nuqui e Phadke (2005).

Zhou et al. (2006) desenvolveram uma estratégia para lidar com medidas de PMUs diretamente no modelo de estimação não linear tradicional, ao considerarem medidas fasoriais de tensão e de corrente em coordenadas retangulares. Paralelamente os autores propuseram também um modelo muito semelhante ao sugerido por Zhao, onde chega-se à conclusão que os resultados obtidos com a aplicação da etapa adicional de estimação linear são compatíveis aos do estimador não linear híbrido. Um estudo comparativo entre vários modelos nessa linha é apresentado em Chakrabarti et al. (2010).

O maior problema relacionado ao uso de coordenadas retangulares é a determinação da covariância dos erros de medição das grandezas transformadas, que, se não tratados, deixam de ser não correlacionados. Bez e Simões-Costa (2012) propõem a aplicação de um estimador ortogonal para solucionar esta questão, de forma que as propriedades estatísticas das medidas são mantidas. Em Simões-Costa, Albuquerque e Bez (2013) aplica-se a teoria da fusão de dados multisensores para combinar de forma ótima estimativas independentes obtidas pelos sistemas SCADA e SMFS, de maneira que este problema é evitado.

\subsubsection{Aplicação a sistemas interconectados}

A primeira menção de PMUs no contexto de EEMA encontra-se no trabalho de Zhao e Abur (2005), cuja formulação foi apresentada na Seção 2.2.2. Na metodologia proposta, sincrofasores são utilizados na etapa de coordenação do estimador de dois níveis para o incremento da redundância nas regiões de fronteira. Esta linha também é seguida por Jiang, Vittal e Heydt (2007), 
onde é proposto ainda um algoritmo de alocação destas medidas para a minimização dos erros de estimação. Neste mesmo método, barras associadas a PMUs são tomadas como referências angulares em cada área, de forma que o problema de assincronismo entre subsistemas já não é mais considerado na etapa secundária de estimação.

O uso de PMUs para a sincronização interárea é uma proposta também seguida por Lakshminarasimhan e Girgis (2007) e por Patel e Girgis (2007), onde demonstra-se a eficácia da utilização de medidas fasoriais em barras de fronteira para mitigação dos erros de estimação em linhas curtas e de baixa impedância. Patel e Girgis também consideram que sincrofasores são mais precisos que medidas convencionais processadas pelo sistema SCADA, especialmente quando estratégias eficientes de calibração de TPs e TCs são tomadas. Dentro desse mesmo contexto, o método desenvolvido por Jiang, Vittal e Heydt no âmbito da teoria diacóptica assume que barras com unidades de medição fasorial são barras de referência, para fins de sincronização interárea e como forma de garantir que certas matrizes na formulação interna sejam inversíveis.

A metodologia discutida na presente tese, com resultados preliminares propostos em Ângelos e Asada (2012), envolve a aplicação do conceito de sincrofasores ao cálculo de Equivalentes Externos. Mostra-se que, caso PMUs sejam instalados em barras de fronteira, importantes propriedades do equivalente Ward serão satisfeitas, trazendo para estimadores multiárea precisão e robustez diante de ocorrências externas. Este assunto é abordado em mais detalhes no próximo Capítulo. 


\section{Capítulo 4}

\section{Equivalentes Externos em Sistemas Elétricos de Potência}

Do ponto de vista da operação, um SEP subdivide-se em duas regiões, a interna, representada pelo próprio sistema sob jurisdição, e a externa, que envolve todas as demais áreas não monitoradas pelo centro de controle, como ilustrado na Fig. (4.1). Barras de fronteira (identificadas pela letra F) fazem a conexão interárea.

Informações em tempo real adquiridas pelo sistema SCADA e SMFS são suficientes para garantir a construção e operacionalização do modelo interno. No entanto, como dados correntes geralmente não estão disponíveis para a parte não observável do sistema, um modelo externo apropriado deverá ser estabelecido, especialmente para a execução de aplicações avançadas relacionadas à segurança do sistema.

Estimativas em barras de fronteira, bem como dados sobre previsão de carga e despacho de geradores das redes vizinhas, podem ser utilizados para a elaboração de um modelo do tipo não reduzido (DURAN; ARVANITIDIS, 1972), baseando-se em Fluxo de Carga ou Estimação de Estado, onde a região externa será representada de forma completa. A vantagem desta estratégia é que informações como intercâmbio de potência entre áreas, barras externas com injeções nulas, dentre outras, podem ser diretamente incorporadas ao problema. Entretanto, em grande parte dos casos, diante da pouca disponibilidade de dados externos confiáveis, uma solução comum é a determinação de um modelo externo reduzido, ou Equivalente Externo (EQE), que será então acoplado ao sistema interno em barras de fronteira, sendo assegurado que sua incorporação não irá alterar o estado estimado interno. Modelos nesta categoria devem ser atualizados em tempo real diante de flutuações no ponto de operação do sistema, procedimento conhecido como ajuste de fronteira. 


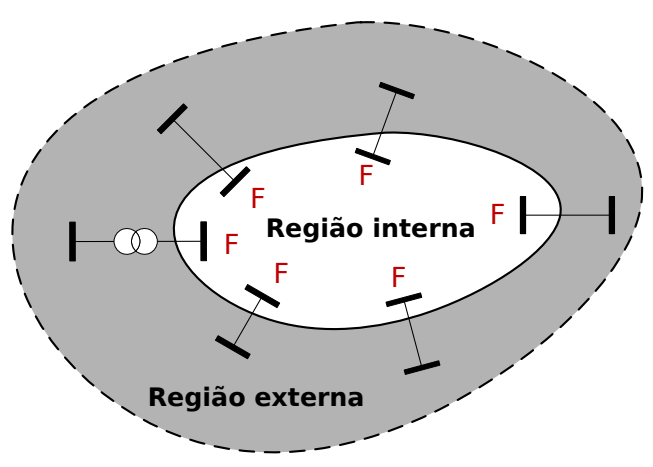

Figura 4.1 - Divisões de um Sistema Elétrico de Potência.

Os modelos de EQEs mais difundidos são os do tipo REI e do tipo Ward $^{1}$. Embora argumentase que uma representação explícita da rede externa possa fornecer resultados mais precisos na análise de faltas ou ocorrências no sistema, mostra-se que a influência de erros na modelagem externa é compensada desde que o ajuste de fronteiras seja efetuado corretamente (DECKMANN et al., 1980a; DECKMANN et al., 1980b). Constata-se que este ajuste nos métodos REI, em geral, é aplicado às custas de alterações nos parâmetros internos do modelo equivalente já construído. Para fins de incorporação de EQEs ao problema de EEMA, proposta desta tese, métodos do tipo Ward são preferíveis, por poderem ser atualizados em tempo real. As demais seções deste Capítulo abordam esta classe de equivalentes e suas variações.

\subsection{Equivalentes Externos do tipo Ward}

Equivalentes do tipo Ward (WARD, 1949) visam representar uma rede elétrica não monitorada por um sistema reduzido formado por circuitos equivalentes e por um conjunto de injeções adicionais em barras de fronteira. Os circuitos equivalentes estarão associados à parte passiva da rede de interesse, ou seja, às linhas e transformadores. As injeções, por outro lado, representarão a carga e geração externa.

No equivalente Ward linear, a rede original é modelada em termos de injeções de corrente, onde a matriz admitância nodal $(\mathbf{Y})$, o vetor de tensões nodais $(\mathbf{E})$ e o vetor de injeções de

\footnotetext{
${ }^{1}$ Análises críticas sobre diferentes estratégias para modelagem de redes externas podem ser vistas em Wu e Monticelli (1983) e em Bose (1984).
} 
corrente (I) estão relacionadas por:

$$
\mathbf{Y E}=\mathbf{I}
$$

Assumindo o SEP constituído por três regiões, interna (índice $i$ ), fronteira (índice $f$ ) e externa (índice e), o modelo da Eq. (4.1) pode ser particionado da seguinte forma:

$$
\left[\begin{array}{c:c:c}
\mathbf{Y}_{\mathrm{ee}} & \mathbf{Y}_{\mathrm{ef}} & \mathbf{0} \\
\hdashline \mathbf{Y}_{\mathrm{fe}} & \mathbf{Y}_{\mathrm{ff}} & \mathbf{Y}_{\mathrm{fi}} \\
\hdashline 0 & \mathbf{Y}_{\mathrm{if}} & \mathbf{Y}_{\mathrm{ii}}
\end{array}\right] \cdot\left[\begin{array}{c}
\mathbf{E}_{\mathrm{e}} \\
-\mathbf{E}_{\mathbf{f}} \\
\mathbf{E}_{\mathrm{i}}
\end{array}\right]=\left[\begin{array}{c}
\mathbf{I}_{\mathrm{e}} \\
\mathbf{I}_{\mathbf{f}} \\
\mathbf{I}_{\mathbf{i}}
\end{array}\right]
$$

Define-se $n_{b i}, n_{b f}$ e $n_{b e}$ como os números de barras internas, de fronteira e externas. O equivalente Ward é obtido fatorando-se os elementos do triângulo inferior da matriz Y por meio de eliminação de Gauss. Este processo implicará na eliminação da variável $\mathbf{E}_{\mathbf{e}}$, que corresponde às tensões nodais das barras externas, e, consequentemente, na obtenção da matriz nodal reduzida $\left(\mathbf{Y}_{\mathbf{f f}}^{\mathbf{e q}}\right)$ e do vetor de injeções equivalentes em barras de fronteira $\left(\mathbf{I}_{\mathbf{f}}^{\mathbf{e q}}\right)$, como representado na Eq. (4.3):

$$
\left[\begin{array}{ccc} 
& \times & \times \\
\hdashline & \mathbf{Y}_{\mathrm{ff}}^{\mathrm{eq}} & \mathbf{Y}_{\mathrm{fi}} \\
\mathbf{0} & \mathbf{Y}_{\mathrm{if}} & \mathbf{Y}_{\mathrm{ii}}
\end{array}\right] \cdot\left[\begin{array}{c}
\mathbf{E}_{\mathrm{e}} \\
\hdashline \mathbf{E}_{\mathbf{f}} \\
\hdashline \mathbf{E}_{\mathbf{i}}
\end{array}\right]=\left[\begin{array}{c}
\times \\
\mathbf{I}_{\mathrm{f}}^{\mathrm{eq}} \\
-\mathbf{I}_{\mathbf{i}}
\end{array}\right]
$$

em que

$$
\begin{gathered}
Y_{f f}^{e q}=Y_{f f}-Y_{f e} Y_{e e}^{-1} Y_{e f} \\
I_{f}^{e q}=I_{f}-Y_{f e} Y_{e e}^{-1} I_{e}
\end{gathered}
$$

A matriz $\mathbf{Y}_{\mathbf{f f}}^{\mathbf{e q}}\left(n_{b f} \times n_{b f}\right)$ é formada por admitâncias existentes na rede original (representando conexões entre barras do tipo fronteira-fronteira e fronteira-interna) e por uma parcela que aparece no decorrer do processo de eliminação, associada à rede externa. Já o vetor $\mathbf{I}_{\mathbf{f}}^{\mathbf{e q}}\left(n_{b f} \times 1\right)$ é constituído por injeções originalmente existentes e uma componente associada à distribuição das injeções externas na fronteira reduzida. Ao final do processo, o modelo equivalente resultante é 

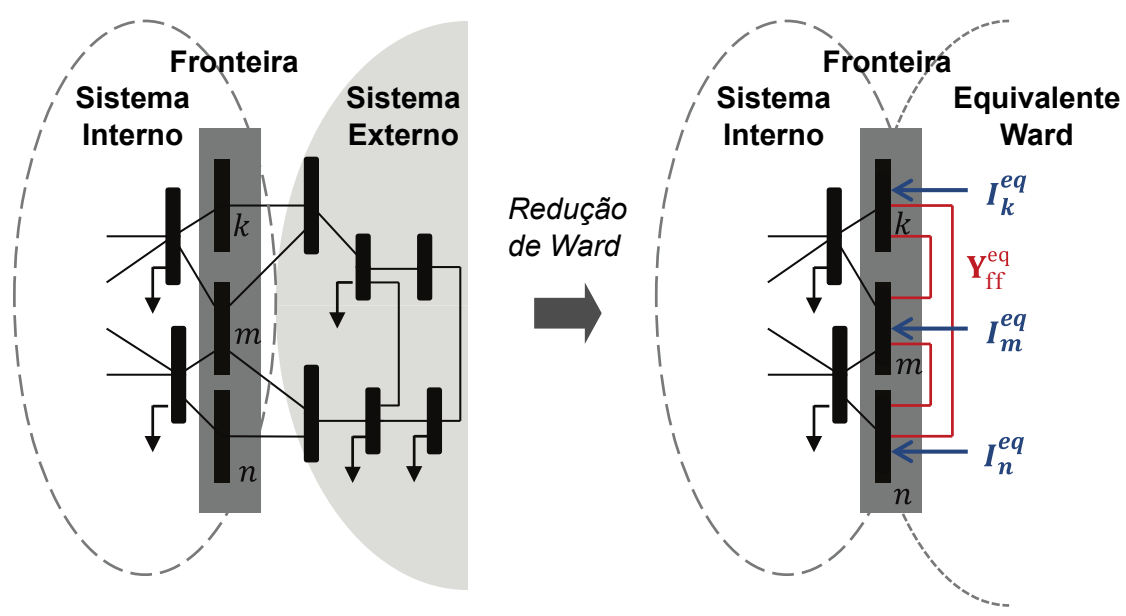

Figura 4.2 - Equivalente Externo Ward Linear

acoplado à rede interna pelas barras de fronteira, como ilustrado na Fig. (4.2).

A Equação (4.5) é pouco viável à aplicação em tempo real por necessitar de injeções de corrente em barras externas. Outra forma mais utilizada, que pode ser obtida a partir de (4.3) é dada por:

$$
\mathbf{I}_{\mathbf{f}}^{\mathrm{eq}}=\mathbf{Y}_{\mathrm{ff}}^{\mathrm{eq}} \mathbf{E}_{\mathrm{f}}+\mathbf{Y}_{\mathrm{fi}} \mathbf{E}_{\mathrm{i}}
$$

Considerando esta última equação, a injeção de corrente equivalente em uma barra de fronteira $k$, será expressa por:

$$
I_{k}^{e q}=-y_{k}^{s h} E_{k}+\sum_{m \in \Omega_{k}^{\mathrm{f}}} y_{k m}^{e q} E_{k m}+\sum_{m \in \Omega_{k}^{\mathrm{i}}} y_{k m} E_{k m}
$$

onde $m$ é uma barra vizinha a $k$, pertencente ao conjunto de barras de fronteira $\left(\Omega_{k}^{\mathbf{f}}\right)$ ou ao conjunto de barras internas $\left(\Omega_{k}^{\mathbf{i}}\right)$; e $y_{k m}^{e q}$ representa a admitância equivalente entre $k$ e $m$.

Segundo a Eq. (4.6), injeções equivalentes podem ser determinadas diretamente a partir das tensões nas barras de fronteira $\left(\mathbf{E}_{\mathbf{f}}\right)$ e internas $\left(\mathbf{E}_{\mathbf{i}}\right)$, que são conhecidas pelo sistema de interesse, sendo obtidas pelo Estimador de Estado interno.

Para um SEP representado pelo modelo de Fluxo de Carga linear, ou seja,

$$
\mathbf{B}^{\prime} \boldsymbol{\theta}=\mathbf{P}
$$

as mesmas considerações das Equações (4.1) a (4.6) são válidas. Nesse caso, a matriz B' equiva- 
lente em barras de fronteira será dada por:

$$
\mathbf{B}_{\mathrm{ff}}^{\prime \text { eq }}=\mathbf{B}_{\mathrm{ff}}^{\prime}-\mathbf{B}_{\mathrm{fe}}^{\prime} \mathbf{B}_{\mathrm{ee}}^{\prime-1} \mathbf{B}_{\mathrm{ef}}^{\prime}
$$

Injeções equivalentes serão de potência ativa, que poderão ser determinadas, para a barra de fronteira $k$, pela expressão (4.10):

$$
P_{k}^{e q}=\sum_{m \in \Omega_{k}^{\mathrm{f}}} \theta_{k m} / x_{k m}^{e q}+\sum_{m \in \Omega_{k}^{\text {io }}} \theta_{k m} / x_{k m}
$$

em que $\theta_{k m}$ denota a defasagem angular entre as barras $k$ e $m$.

\subsubsection{Modelo Ward não linear}

O modelo Ward para sistemas representados por equações não lineares é obtido de forma similar ao que foi exposto. Circuitos equivalentes são igualmente determinados por eliminação gaussiana na matriz Admitância Nodal do sistema original. O procedimento difere no cálculo das injeções equivalentes em barras de fronteira, que serão do tipo potência complexa.

Para a dedução das expressões correspondentes, define-se inicialmente a matriz Admitância Nodal Equivalente ( $\left.\mathbf{Y}^{\mathbf{e q}}\right)$, a partir de (4.3), da seguinte forma:

$$
\mathbf{Y}^{\mathbf{e q}}=\left[\begin{array}{c:c}
\mathbf{Y}_{\mathrm{ff}}^{\mathrm{eq}} & \mathbf{Y}_{\mathrm{fi}} \\
\hdashline \mathbf{Y}_{\mathrm{if}} & \mathbf{Y}_{\mathrm{ii}}
\end{array}\right]
$$

A Eq. (4.7) poderá então ser reescrita como:

$$
I_{k}^{e q}=Y_{k k}^{e q} E_{k k}+\sum_{m \in \Omega_{k}} Y_{k m}^{e q} E_{k m}=\sum_{m \in K} Y_{k m}^{e q} E_{k m}
$$

onde $K$ é o conjunto de barras internas e de fronteira vizinhas à barra $k^{2}$. Considerando então que $Y_{k m}^{e q}=G_{k m}^{e q}+j B_{k m}^{e q}$ e que $E_{m}=V_{m} e^{j \theta_{m}}$, esta última expressão pode ser novamente reescrita como:

$$
I_{k}^{e q}=\sum_{m \in K}\left(G_{k m}^{e q}+j B_{k m}^{e q}\right)\left(V_{m} e^{j \theta_{m}}\right)
$$

\footnotetext{
${ }^{2}$ Notar que barras externas não estão presentes no circuito equivalente.
} 
Logo, assumindo que $E_{k}^{*}=V_{k} e^{-j \theta_{k}}$, o conjugado da potência complexa na barra $k\left(S_{k}^{*}\right)$ será:

$$
S_{k}^{*}=E_{k}^{*} I_{k}=V_{k} e^{-j \theta_{k}} \sum_{m \in K}\left(G_{k m}^{e q}+j B_{k m}^{e q}\right)\left(V_{m} e^{j \theta_{m}}\right)
$$

Finalmente, identificando-se as partes real e imaginária da Eq. (4.14), e considerando que as tensões complexas $E_{k}$ e $E_{m}$ são fornecidas pelo estimador de estado interno, isto é, $E_{k}=\hat{E}_{k}=$ $\hat{V}_{k} e^{-j \hat{\theta}_{k}}$ e $E_{m}=\hat{E}_{m}=\hat{V}_{m} e^{-j \hat{\theta}_{m}}$, as expressões das potências ativa e reativa equivalentes serão dadas por:

$$
\begin{aligned}
P_{k}^{e q} & =\hat{V}_{k} \sum_{m \in K} \hat{V}_{m}\left(G_{k m}^{e q} \cos \hat{\theta}_{k m}+B_{k m}^{e q} \operatorname{sen} \hat{\theta}_{k m}\right) \\
Q_{k}^{e q} & =\hat{V}_{k} \sum_{m \in K} \hat{V}_{m}\left(G_{k m}^{e q} \cos \hat{\theta}_{k m}-B_{k m}^{e q} \operatorname{sen} \hat{\theta}_{k m}\right)
\end{aligned}
$$

As injeções equivalentes de potência ativa e reativa nas barras de fronteira asseguram que o estado do sistema interno após o acoplamento do equivalente externo permanecerá inalterado. Este procedimento, denominado boundary matching (ajuste ou casamento de fronteira) é parte fundamental de um modelo de EQE. A forma relativamente simples como o processo é efetuado nos métodos Ward, sem a necessidade de modificação dos parâmetros da rede equivalente, torna este tipo de representação um dos mais utilizados em centros de controle. Desde que o estado interno seja estimado corretamente, erros na topologia externa transmitidos aos circuitos equivalentes serão compensados pelas injeções (4.15) e (4.16) (MONTICELLI et al., 1979).

\subsubsection{Reação externa e Modelo Ward Estendido}

As tarefas de monitoração das condições atuais da rede elétrica pelo centro de controle visam, sobretudo, garantir a segurança do sistema. Este conceito está relacionado à capacidade de um SEP permanecer operando dentro de limites confiáveis frente à distúrbios iminentes (ou contingências), como saídas de linhas ou geradores (STOTT; ALSAC; MONTICELLI, 1987). A função análise de contingências envolve a simulação de cenários futuros e a listagem daqueles que resultam em maiores violações operacionais, de modo a reforçar previamente a confiabilidade da rede.

Em um sistema interconectado, uma contingência na rede interna perturbará as condições operacionais dos sistemas vizinhos, que, por sua vez, influenciarão significativamente a rede onde a contingência ocorreu. Este fenômeno, conhecido como reação ao sistema externo, pode ser observado como alterações nos fluxos do sistema externo para o interno nas barras de fronteira, e deverá ser previsto com a maior exatidão possível pelos modelos de EQEs. 
Verifica-se que, diante de uma contingência, o método Ward convencional apresenta resultados comparáveis ao sistema completo (não equivalenciado) especialmente na parte ativa do modelo. Todavia, a carência de um tratamento adequado para elementos shunt e barras de tensões controladas (barras PV), que são eliminadas no processo de redução, acarreta erros consideráveis na parte reativa do modelo. O método Ward Estendido (MONTICELLI et al., 1979) busca mitigar esse problema através da adição de um suporte reativo extra a cada barra de fronteira $k$, em resposta a uma alteração $\Delta V_{k}$ na magnitude de tensão, da seguinte forma:

$$
\Delta Q_{k}=V_{k} \hat{B}_{k} \Delta V_{k}
$$

onde $\hat{B_{k}}$ é modelado como uma admitância de um ramo conectado a uma barra PV fictícia, ou como a susceptância de um elemento shunt conectado à barra $k$. Seu valor é computado de forma a compensar a resposta reativa das barras PVs eliminadas, que pode ser obtida a partir do modelo Ward $P V^{3}$, sendo dado por:

$$
\hat{B_{k}}=-\sum_{j \in V} B_{k j}^{w v}
$$

Em outras palavras, $\hat{B}_{k}$ equivale à soma de todas as susceptâncias dos ramos conectando a barra de fronteira $k$ às barras $\mathrm{PVs}$ externas $j$, dentro do modelo Ward PV.

Em suma, os seguintes passos são necessários para a construção do modelo Ward Estendido:

(i) Aplicação de Eliminação de Gauss na matriz admitância nodal completa, para a eliminação dos nós externos, obtendo a matriz $\mathbf{Y}_{\mathbf{f f}}^{\mathbf{e q}}$ e os circuitos equivalentes, segundo a Eq. (4.4). Taps são incluídos com valor unitário e shunts externos (de linhas e de barras) são desconsiderados;

(ii) Cálculo das injeções equivalentes de potência ativa (4.15) e reativa (4.16) no ponto de operação corrente, obtido pelo Estimador de Estado;

(iii) A partir da matriz admitância nodal original, considerando shunts e aterrando barras PVs externas, determinar a matriz $\mathbf{B}^{\prime \prime}$ do FCDR, aplicando então eliminação gaussiana para a obtenção dos shunts equivalentes nas barras de fronteira;

(iv) Inclusão dos elementos shunt ao modelo, como susceptâncias de valor $\hat{B}_{k}$ em barras PV fictícias, ou de valor $\hat{B}_{k} / 2$ em shunts fictícios, acoplando por fim o EQE ao sistema interno.

\footnotetext{
${ }^{3} \mathrm{O}$ método Ward PV é estabelecido aplicando-se eliminação gaussiana na matriz $\mathbf{B}^{\prime \prime}$ do subproblema reativo associado ao FCDR.
} 


\section{Capítulo 5}

\section{Estimação de Estado Descentralizada considerando Modelos Externos}

A existência de redes elétricas cada vez mais interligadas demanda a aplicação de modelos em tempo real apropriados às novas arquiteturas e sistemas de medição presentes. Na busca da melhor forma de modelagem de áreas não observáveis, a Estimação de Estado Multiárea envolve métodos que mantêm uma relação custo-benefício entre a qualidade dos resultados e a complexidade computacional envolvida. O tratamento de redes externas é relegado a uma etapa adicional, executada por um agente independente, ou embutido nos processos iterativos locais mediante a exigência de canais de comunicação entre áreas.

Bose e Clements (1987) afirmam que o problema da correta representação de dados para modelagem externa é similar ao problema de Estimação de Estado Hierárquica. Nesse sentido, Geisler e Bose (1983) propõem o uso de dois estimadores independentes para os sistemas interno e externo. Fatores de ponderação são utilizados para a aplicação de restrições operacionais do sistema externo, que são integradas como pseudomedidas. Já Monticelli e Wu (1985a) sugerem um único modelo de estimação, utilizando o conceito de medidas dormentes ${ }^{1}$ para que dados da rede externa não venham a influenciar nos resultados internos.

Esta tese pretende fornecer um tratamento integrado de EQEs em EEMA, dada à crescente disponibilidade de medidas fasoriais sincronizadas nos SGEs modernos. Considera-se, portanto, neste Capítulo, um SEP composto por subáreas independentes conectadas por ramos de interconexão (linhas de transmissão ou transformadores). O particionamento pode ser estabelecido mediante qualquer critério, como geográfico, operacional ou por nível de tensão, desde que as

\footnotetext{
${ }^{1}$ Uma medida dormente é aquela que não possui influência nos resultados do estado estimado.
} 


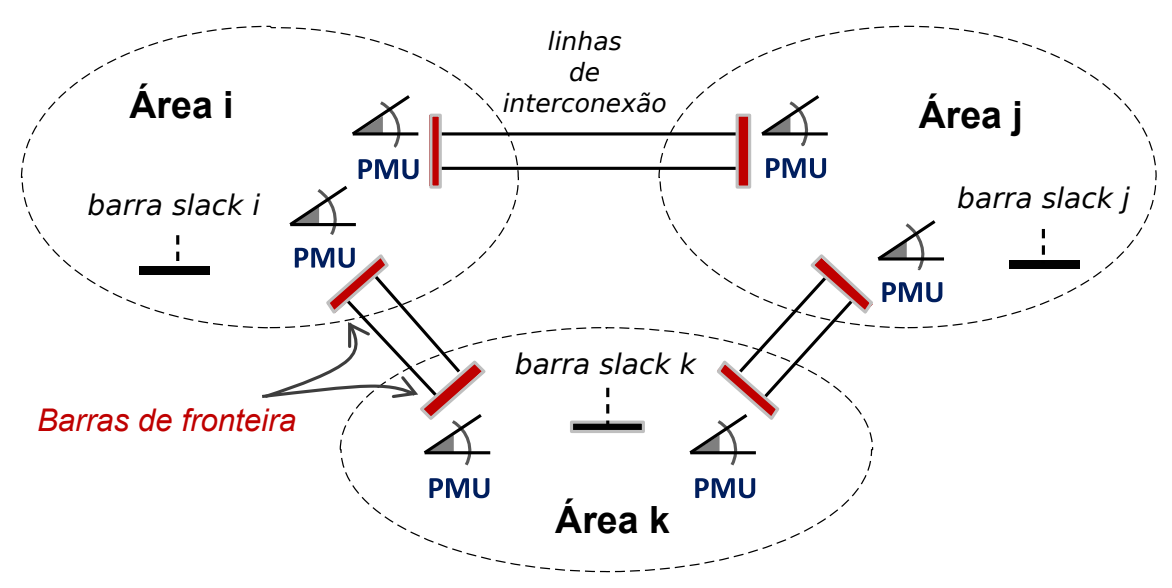

Figura 5.1 - Organização do sistema multiárea

áreas sejam autônomas. É assumido também que PMUs estão alocados em barras de fronteira, provendo medidas fasoriais sincronizadas de tensão e corrente, e que cada área possui sua própria barra de referência, como ilustrado na Fig. (5.1).

Neste tipo de decomposição, barras dos tipos interna, externa e de fronteira são delimitadas, conforme abordado na Seção (2.2). As semelhanças entre este modelo de partição e a organização funcional de EQEs, ilustrado na Fig. (4.2) do Capítulo anterior, sugerem que uma representação externa pode ser incorporada diretamente aos sistemas locais, antes mesmo da etapa de EE. Como visto, EQEs do tipo Ward podem ser atualizados para corresponderem ao ponto de operação corrente do sistema, por meio do ajuste de fronteiras, e poderiam ser utilizados para esta tarefa. No entanto, como o estado atual só é conhecido após a execução dos estimadores, os dados necessários para o ajuste de fronteiras não estarão disponíveis antes desta etapa.

Esta tese propõe o uso de sincrofasores de tensão e corrente, medidos por PMUs em barras de fronteira, para que o ajuste de EQEs, em cada área, seja executado em tempo real, antes da fase de EE. Desta forma, a solução do problema de EEMA pode ser atingida sem a necessidade de uma etapa de centralização, mediante custo computacional e tráfego de dados desprezíveis. A proposta representa uma mudança no processo de construção do modelo de uma rede elétrica em tempo real, no sentido que informações referentes a EQEs poderão ser diretamente incorporadas pelo processo de EE, conforme modelo ilustrado na Fig (5.2). Uma consequência direta deste fato é que dados externos (injeções equivalentes) não compatíveis com o modelo de medição assumido poderão ser detectados e descartados em tempo real.

A descrição do modelo de EEDC objeto desta tese se dará em duas etapas, a definição do modelo em tempo real associado a equivalentes externos e a incorporação do novo modelo ao problema de EE. 


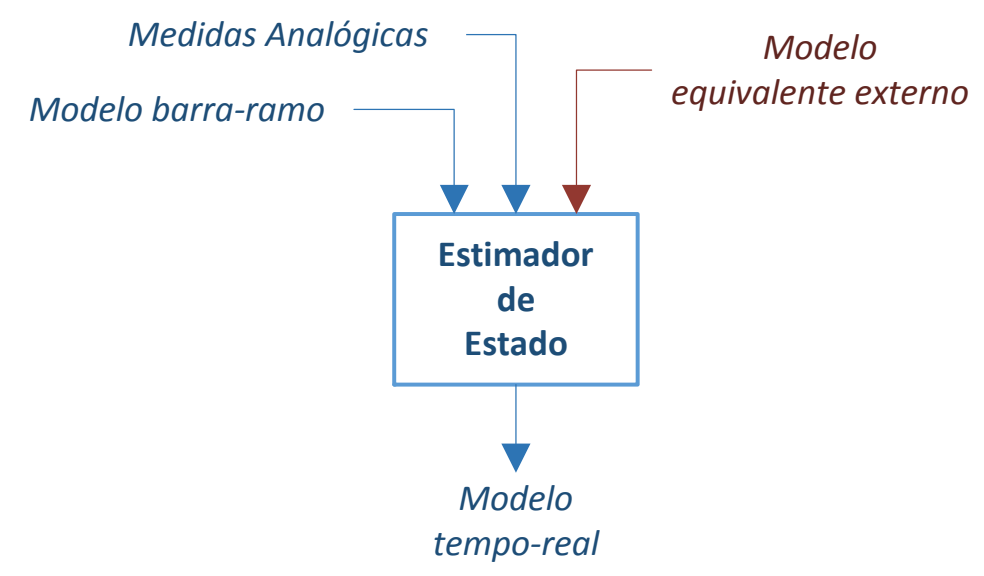

Figura 5.2 - Modelagem em tempo real com Equivalentes Externos incorporados ao Estimador.

\subsection{Definição do modelo das redes externas}

Suponha que um modelo equivalente Ward deva ser criado para cada área do sistema interconectado ilustrado na Fig. (5.1), antes do processamento dos estimadores de estado. Como estudado no Capítulo 4, os circuitos do equivalente Ward podem ser obtidos a partir de eliminação gaussiana na matriz admitância nodal completa (Eq. (4.4)), desde que dados da topologia externa estejam disponíveis. Desta forma determina-se a matriz $\mathbf{Y}_{\mathbf{f f}}^{\mathbf{e q}}$, formada pelas admitâncias equivalentes. O mesmo raciocínio pode ser utilizado para a obtenção do suporte reativo (shunts equivalentes) do modelo Ward Estendido.

O cálculo das injeções equivalentes, no entanto, requer o conhecimento prévio do estado na fronteira, como observado nas Equações (4.15) e (4.16). Esta tese propõe o uso de sincrofasores de tensão e corrente para a execução desta tarefa, mediante a alocação de PMUs em barras de fronteira, de maneira que as tensões requeridas para a atualização do EQE Ward estarão disponibilizadas em tempo real. Desta forma, as equações de injeção de potência equivalente poderão ser reescritas como:

$$
\begin{aligned}
& P_{k}^{e q}=V_{k}^{m e d} \sum_{m \in K} V_{m}^{m e d}\left(G_{k m}^{e q} \cos \theta_{k m}^{m e d}+B_{k m}^{e q} \operatorname{sen} \theta_{k m}^{m e d}\right) \\
& Q_{k}^{e q}=V_{k}^{m e d} \sum_{m \in K} V_{m}^{m e d}\left(G_{k m}^{e q} \cos \theta_{k m}^{m e d}-B_{k m}^{e q} \operatorname{sen} \theta_{k m}^{m e d}\right)
\end{aligned}
$$

onde $V_{k}^{\text {med }} \angle \theta_{k}^{\text {med }}$ e $V_{m}^{\text {med }} \angle \theta_{m}^{\text {med }}$ representam, respectivamente, os sincrofasores de tensão medidos na barra de fronteira $k$ e na barra $m$, que pertence ao conjunto das barras internas e de fronteira 
vizinhas à barra $k$. Se uma determinada barra $m$, por exemplo, do sistema interno, não estiver diretamente monitorada, o sincrofasor de corrente $I_{k m}^{m e d} \angle \alpha_{k m}^{m e d}$, obtido também pelas PMUs, pode ser usado para calcular (pela lei de Kirchoff) o sincrofasor de tensão correspondente. O cálculo de pseudomedidas de tensão pode ser evitado notando-se que as componentes dos fluxos relacionados aos ramos internos nas Equações (5.1) e (5.2) podem ser tomadas diretamente a partir de medidas de fluxo convencionais.

Esta proposta de construção de EQEs promove um novo tipo de informação a ser incorporada na modelagem de SEPs, associada a dados em tempo real sobre redes externas. O novo tipo de informação será referido por Modelo de Equivalente Externo, sendo representado, neste caso, pelo seguinte conjunto de dados:

$$
\Omega=\left\{\mathbf{Y}_{\mathbf{f f}}^{\mathbf{e q}}, \hat{\mathbf{b}}_{\mathbf{f}}^{\mathbf{e q}}, \mathbf{P}_{\mathbf{f}}^{\mathbf{e q}}, \mathbf{Q}_{\mathbf{f}}^{\mathbf{e q}}\right\}
$$

onde $\hat{\mathbf{b}}_{\mathbf{f}}^{\mathbf{e q}}, \mathbf{P}_{\mathbf{f}}^{\mathbf{e q}}, \mathbf{Q}_{\mathbf{f}}^{\mathbf{e q}}$ são vetores de dimensões $n_{b f}$ contendo, respectivamente, elementos equivalentes do tipo shunt, e de injeção de potência ativa e reativa em barras de fronteira. A matriz $\mathbf{Y}_{\mathbf{f f}}^{\mathbf{e q}}$ está definida na Eq. (4.4).

\subsection{Incorporação ao Estimador de Estado}

Assume-se inicialmente que o modelo de medição associado à subárea $a$ será dado por:

$$
\mathbf{z}_{\mathbf{a}}=\mathbf{h}_{\mathbf{a}}\left(\mathbf{y}_{\mathbf{a}}\right)+\mathbf{e}_{\mathbf{z}_{a}}
$$

em que os ângulos do vetor de estado $\mathbf{y}_{\mathbf{a}}$ estão sob a referência angular da barra de referência local, conforme notações definidas na formulação do problema de EEMA (Seção 2.2.2).

Para a incorporação do modelo equivalente externo ao modelo de medição local, nota-se a princípio que, caso o processo de redução da matriz Admitância Nodal seja efetuado pela forma convencional (segundo a Eq. 4.4), medidas de fluxos de potência em linhas de interconexão e de injeções de potência em barras de fronteira deverão ser descartadas. Para evitar esse problema, o procedimento de eliminação gaussiana deve ser finalizado nas barras de primeira vizinhança, como ilustrado na Fig. (5.3).

Desta forma, o vetor de estado local poderá ser expresso por:

$$
\mathbf{y}_{\mathbf{a}}=\left[\begin{array}{lll}
\mathbf{y}_{\mathbf{i} a}^{\prime} & \mathbf{y}_{\mathbf{f} a}^{\prime} & \mathbf{y}_{\mathbf{n} a}^{\prime}
\end{array}\right]^{\prime}
$$

com $\mathbf{y}_{\mathbf{i} a}, \mathbf{y}_{\mathbf{f} a}$ e $\mathbf{y}_{\mathbf{n} a}$ representando, respectivamente, o estado das barras internas, de fronteira e de primeira vizinhança. 


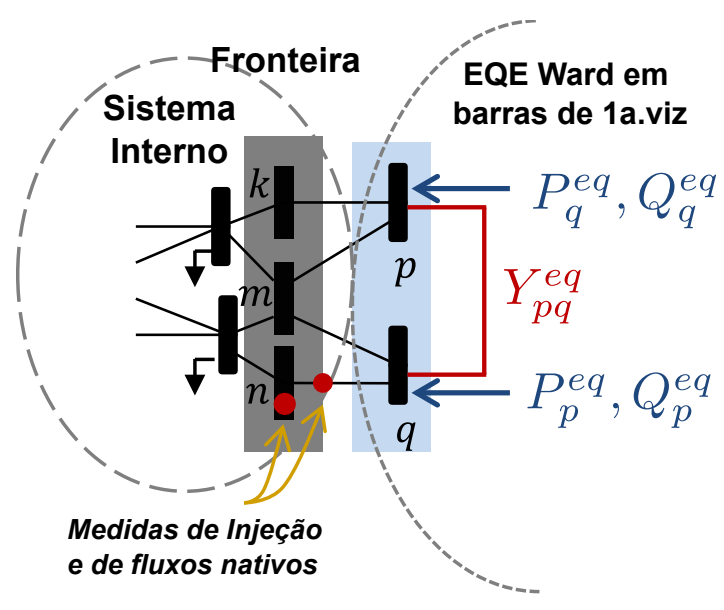

Figura 5.3 - Redução de Ward em barras de primeira vizinhança

Duas partes do modelo equivalente externo devem então ser integradas ao modelo interno, uma relacionada a dados obtidos em uma base de tempo aproximadamente estática (matriz $\mathbf{Y}_{\mathbf{f f}}^{\mathbf{e q}}$ e vetor $\left.\mathbf{b}_{\mathbf{f}}^{\mathbf{e q}}\right)^{2}$ e outra definida em tempo real $\left(\mathbf{P}_{\mathbf{f}}^{\mathbf{e q}}, \mathbf{Q}_{\mathbf{f}}^{\mathbf{e q}}\right)$. A incorporação da primeira é direta, efetuada pela adição dos circuitos e elementos shunt equivalentes. Para a inclusão das injeções equivalentes em tempo real, propõe-se, a seguir, dois modelos, que tratam estas informações como medidas convencionais de alta confiança ou como restrições de igualdade.

\subsubsection{Modelagem como medidas de alta confiança}

Esta estratégia baseia-se na premissa que as injeções equivalentes em tempo real comportamse como medidas de elevada precisão, com variâncias na ordem de grandeza dos sincrofasores utilizados para sua determinação (ÂNGELOS; ASADA, 2012). Assim, o modelo de medição local (Eq. 5.4), integrado ao modelo de medição relacionado ao EQE, será expresso por:

$$
\left[\begin{array}{c}
\mathbf{z}_{\mathbf{a}} \\
\mathbf{z}_{\mathbf{a}}^{\text {eq }}
\end{array}\right]=\left[\begin{array}{c}
\mathbf{h}_{\mathbf{a}}\left(\mathbf{y}_{\mathbf{a}}\right) \\
\mathbf{h}_{\mathbf{a}}^{\mathbf{e q}}\left(\mathbf{y}_{\mathbf{a}}\right)
\end{array}\right]+\left[\begin{array}{c}
\mathbf{e}_{\mathbf{z}_{a}} \\
\mathbf{e}_{\mathbf{z}_{a}}^{\text {eq }}
\end{array}\right]
$$

onde $\mathbf{z}_{\mathbf{a}}^{\text {eq }}$ é o vetor composto pelas injeções de potência ativa e reativa do modelo equivalente externo da área $a ; \mathbf{h}_{\mathbf{a}}^{\mathbf{e q}}$ é o vetor de equações não lineares que relaciona $\mathbf{z}_{\mathbf{a}}^{\mathbf{e q}}$ ao estado $\mathbf{y}_{\mathbf{a}}$. O vetor $\mathbf{e}_{\mathbf{z}_{a}}^{\mathbf{e q}}$, de média zero e matriz covariância $\mathbf{R}_{\mathbf{a}}^{\mathbf{e q}}$, contém os erros gaussianos associados às injeções equivalentes, atribuídos empiricamente com base na precisão das medidas das PMUs.

\footnotetext{
${ }^{2} \mathrm{O}$ índice $f$ denota aqui a fronteira original do sistema ou, conforme a Fig. (5.3), a fronteira estendida para barras de primeira vizinhança.
} 
O problema de MQP associado à Eq. (5.6) envolve encontrar a estimativa $\hat{\mathbf{y}}_{\mathbf{a}}$ que minimiza a seguinte função objetivo:

$$
J_{\mathbf{a}}+J_{\mathbf{a}}^{\mathbf{e q}}=\frac{1}{2} \mathbf{r}_{\mathbf{a}}^{\prime} \mathbf{R}_{\mathbf{a}}^{-1} \mathbf{r}_{\mathbf{a}}+\frac{1}{2} \mathbf{r}_{\mathbf{a}}^{\mathbf{e q}}\left(\mathbf{R}_{\mathbf{a}}^{\mathbf{e q}}\right)^{-1} \mathbf{r}_{\mathbf{a}}^{\mathbf{e q}}
$$

em que $\mathbf{r}_{\mathbf{a}}=\mathbf{z}_{\mathbf{a}}-\mathbf{h}_{\mathbf{a}}\left(\hat{\mathbf{y}}_{\mathbf{a}}\right)$ representa o vetor de resíduos associado às medidas locais da área $a$ e $\mathbf{r}_{\mathbf{a}}^{\mathbf{e q}}=\mathbf{z}_{\mathbf{a}}^{\mathbf{e q}}-\mathbf{h}_{\mathbf{a}}^{\mathbf{e q}}\left(\hat{\mathbf{y}}_{\mathbf{a}}\right)$ denota o vetor de resíduos das injeções de potência equivalentes. A solução deste problema equivale à do modelo básico (Eq. 5.4) considerando medidas equivalentes adicionadas diretamente ao vetor de medidas locais $\left(\mathbf{z}_{\mathbf{a}}\right)$, podendo, portanto, ser processada da forma convencional, conforme Eq. (2.4a) e (2.4b).

Uma vez executados os estimadores das $n_{a}$ áreas do sistema, os estados locais (sob referências angulares distintas) podem ser corrigidos para uma referência comum considerando, por exemplo, as diferenças entre medidas de ângulo de tensão em fronteiras e as correspondentes estimativas de ângulo. Assim, um offset $u_{a}^{\text {sync }}$ poderá ser adicionado aos ângulos estimados da área $a\left(\hat{\boldsymbol{\theta}}_{\mathbf{a}}\right)$ para referenciá-los no sinal global das PMUs, da seguinte forma:

$$
\begin{aligned}
& u_{a}^{\text {sync }}=\sum_{k \in F} \frac{\left(\theta_{k}^{\text {med }}-\hat{\theta}_{k}\right)}{n_{b f}} \\
& \hat{\boldsymbol{\theta}}_{\mathbf{a}}^{\text {sync }}=\hat{\boldsymbol{\theta}}_{\mathbf{a}}+u_{a}^{\text {sync }}
\end{aligned}
$$

onde $k$ pertence ao conjunto de barras de fronteira $(F) ; \theta_{k}^{\text {med }}$ e $\hat{\theta}_{k}$ são, respectivamente, os ângulos de tensão medidos e estimados na barra $k ; n_{b f}$ é o número de barras de fronteira; e $\hat{\boldsymbol{\theta}}_{\mathrm{a}}^{\text {sync }}$ representa o vetor de ângulos de tensão estimados na área $a$ sob a referência angular das PMUs.

Devido à possibilidade da introdução de erros contidos em estimativas de ângulo, uma solução mais precisa para se garantir um sincronismo interárea natural é utilizando as próprias medidas de ângulo como referências locais. Nesse caso, as estimativas obtidas estarão em uma base de tempo e de frequência comuns, associada ao Tempo Universal Coordenado (UTC).

\section{Aplicação ao modelo linearizado}

Para o estimador linearizado, utilizado no exemplo ilustrativo da Seção 5.4, o modelo de medição relacionado a EQEs será dado por:

$$
\begin{gathered}
\mathbf{z}_{\mathbf{p}}^{\mathbf{e q}}=\mathbf{H}_{\mathbf{p}}^{\mathbf{e q}} \boldsymbol{\theta}+\mathbf{e}_{\mathbf{p}}^{\mathbf{e q}} \\
E\left(\mathbf{e}_{\mathbf{p}}^{\mathbf{e q}}\right)=0, E\left(\mathbf{e}_{\mathbf{p}}^{\mathbf{e q}}\left(\mathbf{e}_{\mathbf{p}}^{\mathrm{eq}}\right)^{\prime}\right)=\mathbf{R}_{\mathbf{p}}^{\mathbf{e q}}
\end{gathered}
$$


em que $\mathbf{z}_{\mathbf{p}}^{\mathbf{e q}}$ é o vetor de fluxos e injeções de potência ativa equivalentes, calculadas segundo a Eq. (4.10); $\mathbf{e}_{\mathbf{p}}^{\mathbf{e q}}$ é o vetor de erros aleatórios associados; e $\mathbf{H}_{\mathbf{p}}^{\mathbf{e q}}$ é a matriz Jacobiana (matriz de observação) correspondente.

Assumindo $\mathbf{z}_{\mathbf{p}}=\left[\begin{array}{ll}\mathbf{z}_{\mathbf{p}}^{0^{\prime}} & \mathbf{z}_{\mathbf{p}}^{\mathbf{e q}}\end{array}\right]^{\prime}$ e $\mathbf{e}_{\mathbf{p}}=\left[\begin{array}{ll}\mathbf{e}_{\mathbf{p}}^{0^{\prime}} & \mathbf{e}_{\mathbf{p}}^{\mathbf{e}}{ }^{\prime}\end{array}\right]^{\prime}$, onde $\mathbf{z}_{\mathbf{p}}^{0}$ é o vetor de medidas original (medidas locais) e $\mathbf{e}_{\mathbf{p}}^{0}$ é o vetor de erros aleatórios correspondente, a estimativa local $\hat{\boldsymbol{\theta}}$ poderá ser obtida da forma descrita na Seção 2.1.4, dada por:

$$
\mathbf{G}_{\mathbf{p}} \hat{\boldsymbol{\theta}}=\mathbf{H}_{\mathbf{p}}^{\prime} \mathbf{R}_{\mathrm{p}}^{-1} \mathbf{z}_{\mathrm{p}}
$$

A maior vantagem de integrar injeções de potência como medidas é que rotinas para EE e funções correlatas pré-existentes nos subsistemas, baseadas em MQP, permanecerão inalteradas e completamente funcionais. A desvantagem reside na escolha mais apropriada para os pesos relacionados. Assumindo que PMUs fornecem medidas altamente precisas, valores elevados serão atribuídos, aumentando-se também as possibilidades de problemas numéricos ao longo do processo de solução. Um modelo de integração mais robusto pode ser estabelecido tratando-se dados correntes sobre EQEs como restrições de igualdade no problema de EE, como mostrado a seguir.

\subsubsection{Modelagem como Restrições de Igualdade}

Considera-se inicialmente que medidas de ângulo de tensão advindas de PMUs fornecem a referência angular para as subáreas. Logo, a notação convencional do vetor de estado (x) será utilizada. Havendo-se então incorporada a parte estática do modelo equivalente, o problema de MQP com restrições de igualdade associadas a EQEs em tempo real pode ser formulado como:

$$
\begin{array}{ll}
\text { Minimizar } & J\left(\mathbf{x}_{\mathbf{a}}\right)=\frac{1}{2} \mathbf{r}_{\mathbf{a}}^{\prime} \mathbf{R}_{\mathbf{a}}^{-1} \mathbf{r}_{\mathbf{a}} \\
\text { sujeito a } & \mathbf{P}_{\mathbf{f}}^{(\mathbf{a})}\left(\mathbf{x}_{\mathbf{a}}\right)-\mathbf{P}_{\mathbf{f}}^{(\mathbf{a})_{\mathbf{e q}}}=0 \\
& \mathbf{Q}_{\mathbf{f}}^{(\mathbf{a})}\left(\mathbf{x}_{\mathbf{a}}\right)-\mathbf{Q}_{\mathbf{f}}^{(\mathbf{a})_{\mathbf{e q}}}=0
\end{array}
$$

onde $\mathbf{r}_{\mathbf{a}}=\mathbf{z}_{\mathbf{a}}-\mathbf{h}_{\mathbf{a}}\left(\mathbf{x}_{\mathbf{a}}\right)$ é o vetor de resíduos relacionado às medidas locais da área $a ; \mathbf{P}_{\mathbf{f}}^{(\mathbf{a})}\left(\mathbf{x}_{\mathbf{a}}\right)$ e $\mathbf{Q}_{\mathbf{f}}^{(\mathbf{a})}\left(\mathbf{x}_{\mathbf{a}}\right)$ são, respectivamente, os vetores de injeções de potência ativa e reativa nas barras de fronteira da área $a$; e $\mathbf{P}_{\mathbf{f}}^{(\mathbf{a})_{\mathbf{e q}}}$ e $\mathbf{Q}_{\mathbf{f}}^{(\mathbf{a})_{\mathbf{e q}}}$ são os vetores de injeções equivalentes correspondentes.

A função Lagrangeana correspondente é escrita da seguinte forma:

$$
\mathcal{L}\left(\mathbf{x}_{\mathbf{a}}, \boldsymbol{\lambda}\right)=\frac{1}{2}\left[\mathbf{z}_{\mathbf{a}}-\mathbf{h}_{\mathbf{a}}\left(\mathbf{x}_{\mathbf{a}}\right)\right]^{\prime} \mathbf{R}_{\mathbf{a}}^{-1}\left[\mathbf{z}_{\mathbf{a}}-\mathbf{h}_{\mathbf{a}}\left(\mathbf{x}_{\mathbf{a}}\right)\right]+\boldsymbol{\lambda}^{\prime} \mathbf{c}\left(\mathbf{x}_{\mathbf{a}}\right)
$$


onde $\mathbf{c}\left(\mathbf{x}_{\mathbf{a}}\right)$ é o vetor de equações não lineares com as restrições de injeções de potência da área $a$ (5.13b e 5.13c); e $\boldsymbol{\lambda}$ é o vetor de multiplicadores de Lagrange.

A solução deste problema, discutido na Seção 2.1.3, considera o seguinte processo iterativo:

$$
\left[\begin{array}{cc}
\mathbf{H}_{\mathbf{a}}^{\prime}\left(\mathbf{x}_{\mathbf{a}}^{v}\right) \mathbf{R}_{\mathbf{a}}^{-1} \mathbf{H}_{\mathbf{a}}\left(\mathbf{x}_{\mathbf{a}}^{v}\right) & -\mathbf{C}^{\prime}\left(\mathbf{x}_{\mathbf{a}}^{v}\right) \\
-\mathbf{C}\left(\mathbf{x}_{\mathbf{a}}^{v}\right) & \mathbf{0}
\end{array}\right]\left[\begin{array}{c}
\Delta \mathbf{x}_{\mathbf{a}} \\
\lambda^{v+1}
\end{array}\right]=\left[\begin{array}{c}
\mathbf{H}_{\mathbf{a}}^{\prime}\left(\mathbf{x}_{\mathbf{a}}^{v}\right) \mathbf{R}_{\mathbf{a}}^{-1} \mathbf{r}_{\mathbf{a}}\left(\mathbf{x}_{\mathbf{a}}^{v}\right) \\
\mathbf{c}\left(\mathbf{x}_{\mathbf{a}}^{v}\right)
\end{array}\right]
$$

onde $\boldsymbol{\Delta} \mathbf{x}_{\mathbf{a}}$ é a correção a ser aplicada ao estado $\mathbf{x}_{\mathbf{a}}$ na iteração $v$.

Após o processamento dos estimadores de estado de todas as áreas, a solução do problema de EEMA terá sido encontrada. Como o modelo EQE Ward fornece representações quase exatas dos sistemas externos, as estimativas obtidas com os modelos representados serão também muito precisas.

\subsection{Procedimento Geral}

Os principais passos para o processamento integrado e sincronização de múltiplas áreas proposto são apresentados a seguir.

Passo 1. Aplique Eliminação de Gauss nas barras do sistema externo, determinando a submatriz $\mathbf{Y}_{\mathbf{f f}}^{\mathbf{e q}}$ e os circuitos equivalentes do modelo Ward, segundo a Eq. (4.4);

Passo 2. Efetue o ajuste de fronteiras no ponto de operação corrente, utilizando sincrofasores medidos por PMUs, obtendo os vetores $\mathbf{P}_{\mathbf{f}}^{\mathbf{e q}}$ e $\mathbf{Q}_{\mathbf{f}}^{\mathbf{e q}}$ a partir das Eqs. (5.1) e (5.2);

Passo 3. Para a inclusão do suporte reativo adicional, compute os elementos shunt equivalentes em fronteiras (aplicação do Método Ward Estendido);

Passo 4. Processe o estimador de estado local, integrando injeções equivalentes como medidas (Seção. 5.2.1) ou restrições de igualdade (Seção. 5.2.2);

Passo 5. Efetue a sincronização das áreas (Equações 5.8 e 5.9), caso referências angulares não tenham sido fornecidas por PMUs.

Como alterações na topologia externa ocorrem, normalmente, a uma frequência relativamente baixa, o passo 1 não será executado frequentemente. Nota-se ainda que o efeito de erros nos circuitos equivalentes são eliminados quando as injeções equivalentes são calculadas no estado interno correto, como visto no Capítulo 4. Por fim, verifica-se que a carga computacional associada ao passo 2 é mínima, sendo também beneficiado pelas altas taxas com que medidas de PMUs são fornecidas. 


\subsection{Exemplo Ilustrativo: Sistema IEEE 14 barras com modelo linearizado}

Apresenta-se nesta seção um exemplo de aplicação da metodologia proposta ao sistema IEEE 14 barras (UWEE, 2011) sob um modelo de medição linearizado. A rede está particionada em duas áreas, como ilustrado na Fig. (5.4), em que a primeira é formada pelas barras 1 a 5 e possui referência angular na barra 1, enquanto a segunda compreende as barras 6 a 14 com referência angular na barra 14. O plano de medição global contempla 43 medidas (9 de injeções de potência ativa e 34 de fluxos de potência ativa), que foram geradas adicionando-se erros gaussianos com médias nulas e desvios-padrão de $\sigma=0,01$ p.u aos resultados do fluxo de carga linear. Medidas em fronteiras não são consideradas neste exemplo.

Assume-se que PMUs fornecem medidas de ângulo de tensão nas barras onde estão instalados e de fluxos de potência ativa nas linhas adjacentes, que por sua vez, são utilizadas para o cálculo dos ângulos nas barras vizinhas. Medidas de ângulo são incorporadas com desvios-padrão de $\sigma=0,0067^{\circ}$, isto é, $1 / 3$ de $0,02^{\circ}$, valor aproximado admitido em um sistema de $60 \mathrm{~Hz}$ quando somente erros de sincronismo são considerados ((ZHOU, 2008)), sendo utilizadas somente para o

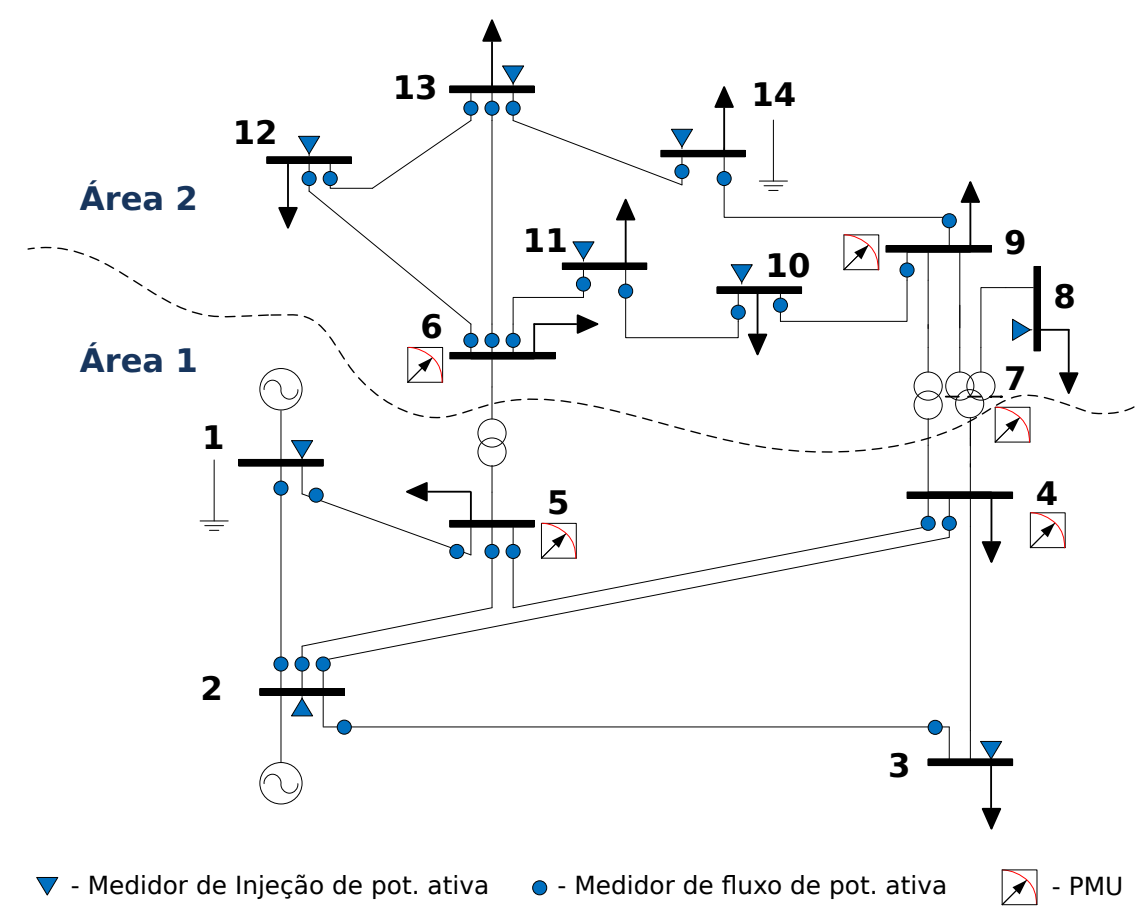

Figura 5.4 - Sistema IEEE-14: configuração das subáreas. 
cálculo das injeções equivalentes. Atribui-se ao ângulo de tensão medido na barra 12 o valor zero, isto é $\theta_{12}^{p m u}=0^{\circ}$. O procedimento para a solução do problema de EEMA é descrito a seguir.

\section{Modelo interno da área 1}

Lista-se na Tab. (5.1) o plano de medição da área 1, que é composto por 3 medidas de injeção de potência, 7 medidas de fluxos do tipo $p_{k m}$ e 7 medidas de fluxos $p_{m k}$. Uma pseudomedida de ângulo de valor zero e desvio padrão $10^{-7}$ também está presente, representando a referência angular do sistema. Na Tabela (5.2) são listadas as medidas fornecidas pelas PMUs: ângulos das tensões nas barras de fronteira 4 e 5 e fluxos nas linhas adjacentes.

Utilizando informações topológicas da área 2 e aplicando-se eliminação de Gauss na matriz $\mathbf{B}^{\prime}$ da rede completa, segundo a Eq. (4.9), é obtido o circuito equivalente do modelo externo $\left(x_{4-5}^{e q}=0,741 \mathrm{p} . \mathrm{u}\right)$. As injeções correspondentes de potência ativa poderão ser obtidas a partir da Eq. (4.10), considerando os ângulos de tensão nas barras de fronteira e os ângulos nas barras vizinhas a elas. Os primeiros são disponibilizados pelas PMUs nas barras 4 e 5. Já os segundos podem ser calculados a partir destes, considerando os fluxos nas linhas adjacentes e as reatâncias nos ramos. ${ }^{3}$ Desta forma, uma pseudomedida de ângulo na barra 2, vizinha à barra de fronteira 4, poderá ser obtida, por exemplo, da seguinte forma:

$$
\theta_{2}^{p s}=\theta_{4}^{p m u}-p_{4-2}^{p m u} * x_{42}=0,100-(-0,555) * 0,176=0,198 \mathrm{rad}
$$

A injeção equivalente de potência ativa na barra 4 será então dada por:

$$
\begin{aligned}
P_{4}^{e q} & =\frac{\theta_{4}^{p m u}-\theta_{2}^{p s}}{x_{24}}+\frac{\theta_{4}^{p m u}-\theta_{3}^{p s}}{x_{34}}+\frac{\theta_{4}^{p m u}-\theta_{5}^{p m u}}{\left(x_{45}^{e q} \| x_{45}\right)} \\
& =\frac{0,100-0,198}{0,176}+\frac{0,100-0,060}{0,171}+\frac{0,100-0,126}{0,040}=-0,9734 p . u
\end{aligned}
$$

Seguindo o mesmo raciocínio, a injeção equivalente $P_{5}^{e q}$ será expressa por:

$$
P_{5}^{e q}=\frac{\theta_{5}^{p m u}-\theta_{1}^{p s}}{x_{15}}+\frac{\theta_{5}^{p m u}-\theta_{2}^{p s}}{x_{25}}+\frac{\theta_{5}^{p m u}-\theta_{4}^{p m u}}{\left(x_{45}^{e q}|| x_{45}\right)}=-0,4715 p . u
$$

Finalmente, o modelo reduzido da área 1 considerando equivalentes incorporados está representado na Fig. (5.5).

\footnotetext{
${ }^{3}$ No caso do estimador não linear, este procedimento será equivalente ao cálculo de pseudomedidas de tensão a partir de medidas fasoriais sincronizadas de tensão e de corrente, considerando a lei de Kirchoff.
} 
Tabela 5.1 - Sistema IEEE-14: plano de medição da área 1

\begin{tabular}{|c|c|c|c|}
\hline Tipo & Valor & Tipo & Valor \\
\hline$p_{1}$ & 2,226 p.u & $p_{4-5}$ & $-0,621$ p.u \\
\hline$p_{2}$ & 0,184 p.u & $p_{2-1}$ & $-14,724$ p.u \\
\hline$p_{3}$ & $-0,934$ p.u & $p_{5-1}$ & $-0,712$ p.u \\
\hline$p_{1-2}$ & 1,485 p.u & $p_{3-2}$ & $-0,703$ p.u \\
\hline$p_{1-5}$ & 0,714 p.u & $p_{4-2}$ & $-0,559$ p.u \\
\hline$p_{2-3}$ & 0,706 p.u & $p_{5-2}$ & $-0,411$ p.u \\
\hline$p_{2-4}$ & 0,552 p.u & $p_{4-3}$ & 0,243 p.u \\
\hline$p_{2-5}$ & 0,410 p.u & $p_{5-4}$ & 0,618 p.u \\
\hline$p_{3-4}$ & $-0,241$ p.u & $\theta_{1}$ & $0 \mathrm{rad}$ \\
\hline
\end{tabular}

Tabela 5.2 - Sistema IEEE-14: medidas fornecidas pelas PMUs (área 1)

\begin{tabular}{c|c|c|c|c|c|c}
\hline Tipo & $\boldsymbol{\theta}_{\mathbf{4}}^{\boldsymbol{p m u}}$ & $\boldsymbol{p}_{\mathbf{4}-\mathbf{2}}^{\boldsymbol{p m u}}$ & $\boldsymbol{p}_{\mathbf{4}-\mathbf{3}}^{\boldsymbol{p m u}}$ & $\boldsymbol{\theta}_{\mathbf{5}}^{\boldsymbol{p m u}}$ & $\boldsymbol{p}_{\mathbf{5}-\mathbf{1}}^{\boldsymbol{p m u}}$ & $\boldsymbol{p}_{\mathbf{5}-\mathbf{2}}^{\boldsymbol{p m u}}$ \\
\hline Valor & $0,100 \mathrm{rad}$ & $-0,555 \mathrm{p.u}$ & $0,240 \mathrm{p.u}$ & $0,126 \mathrm{rad}$ & $-0,716 \mathrm{p} . \mathrm{u}$ & $-0,412 \mathrm{p.u}$ \\
\hline
\end{tabular}

Área 1

Eq. Ward Área 2

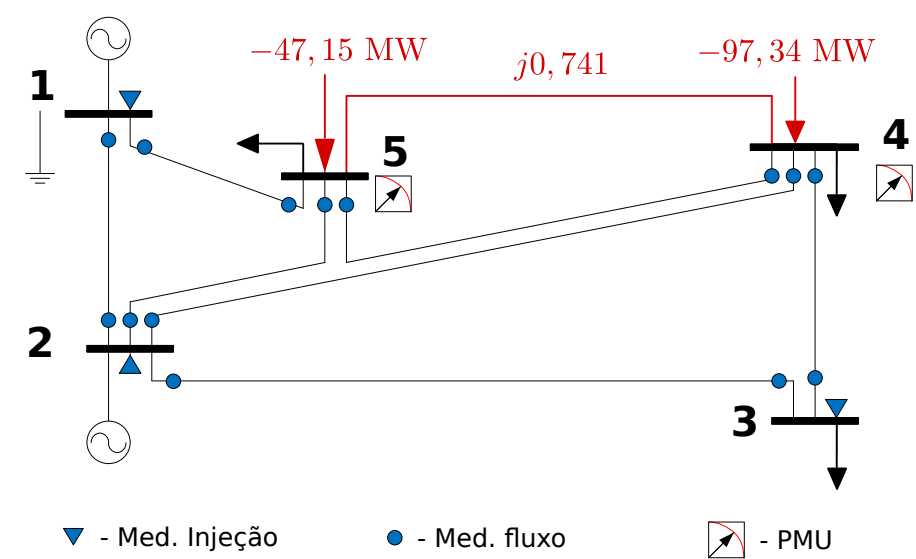

Figura 5.5 - Sistema IEEE-14: modelo reduzido da área 1 


\section{Modelo interno da área 2}

O subsistema 2 é formado por 9 barras e 10 ramos. O plano de medição interno, descrito na Tab. (5.3), contém 6 medidas de injeção, 10 medidas de fluxos $p_{k m}$ e 10 medidas de fluxos $p_{m k}$, além de uma pseudomedida de ângulo na barra 14, referência angular do sistema. Às medidas de injeções com valores nulos são atribuídos desvios-padrão $\sigma=10^{-7}$ p.u. Na Tabela (5.4) listam-se as medidas fasoriais sincronizadas utilizadas para o cálculo dos equivalentes em tempo real.

A partir da configuração topológica da área 1, o equivalente externo é obtido aplicandose Eliminação de Gauss para a eliminação das barras pertencentes à área 1, o que resulta em 3 circuitos equivalentes. Baseando-se nos sincrofasores medidos nas barras 6,7 e 9 , segundo o mesmo procedimento efetuado na área 1, injeções equivalentes são computadas no estado corrente, resultando na rede reduzida mostrada na Fig. (5.6).

\section{Estimação do estado}

Uma vez acoplados os circuitos dos modelos equivalentes, estimativas locais são então determinadas utilizando o modelo linearizado, conforme a Eq. (5.12). Informações sobre injeções equivalentes em tempo real são incluídas com o mesmo desvio padrão assumido para as medidas de injeções nulas, para ressaltar sua elevada precisão. Finalmente, as estimativas são sincronizadas com a referência comum das PMUs em fronteiras, segundo o procedimento descrito nas Eq. (5.8) e (5.9).

Na Tabela (5.5), resultados obtidos com a inclusão de EQEs (metodologia proposta, com valores em negrito) são comparados ao modelo MQP linear convencional. Consideram-se como valores de referência os obtidos pelo EEL executado no sistema completo, mostrados na segunda coluna da Tabela $\left(\hat{\theta}^{r e f}\right)$. Verifica-se que a adição de injeções equivalentes de potência contribui significativamente para a melhoria das estimativas locais, em especial nas barras de fronteira. Testes em outros sistemas, considerando modelos não lineares e com tratamento estatístico mais adequado, são apresentados no próximo Capítulo. 
Tabela 5.3 - Sistema IEEE-14: plano de medição da área 2.

\begin{tabular}{c|c|c|c}
\hline Tipo & Valor $(\mathrm{p} . \mathrm{u})$ & Tipo & Valor $(\mathrm{p} . \mathrm{u})$ \\
\hline$p_{8}$ & 0,000 & $p_{10-11}$ & $-0,029$ \\
$p_{10}$ & $-0,091$ & $p_{12-13}$ & 0,015 \\
$p_{11}$ & $-0,036$ & $p_{13-14}$ & 0,050 \\
$p_{12}$ & $-0,061$ & $p_{11-6}$ & $-0,062$ \\
$p_{13}$ & $-0,133$ & $p_{12-6}$ & $-0,076$ \\
$p_{14}$ & $-0,150$ & $p_{13-6}$ & $-0,169$ \\
$p_{6-11}$ & 0,063 & $p_{8-7}$ & 0,000 \\
$p_{6-12}$ & 0,076 & $p_{9-7}$ & $-0,286$ \\
$p_{6-13}$ & 0,168 & $p_{10-9}$ & $-0,061$ \\
$p_{7-8}$ & 0,000 & $p_{14-9}$ & $-0,099$ \\
$p_{7-9}$ & 0,287 & $p_{11-10}$ & 0,028 \\
$p_{9-10}$ & 0,063 & $p_{13-12}$ & $-0,014$ \\
$p_{9-14}$ & 0,098 & $p_{14-13}$ & $-0,049$ \\
\hline
\end{tabular}

Tabela 5.4 - Sistema IEEE-14: medidas fornecidas pelas PMUs (área 2)

\begin{tabular}{|c|c|c|c|}
\hline Tipo & Valor & Tipo & Valor \\
\hline$\theta_{6}^{p m u}$ & $0,019 \mathrm{rad}$ & $p_{7-8}^{p m u}$ & 0,001 p.u \\
\hline$p_{6-11}^{p m u}$ & $0, \mathrm{C}$ & $\theta_{9}^{p m u}$ & $007 \mathrm{rad}$ \\
\hline$p_{6-12}^{p m u}$ & 0,075 & $p_{9-10}^{p m u}$ & 0,084 p.u \\
\hline$p_{6-13}^{p m u}$ & $0,170 \mathrm{p}$ & $p_{9-14}^{p m u}$ & 0,270 p.u \\
\hline$\theta_{7}^{p m u}$ & $0,039 \mathrm{rad}$ & 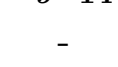 & - \\
\hline
\end{tabular}




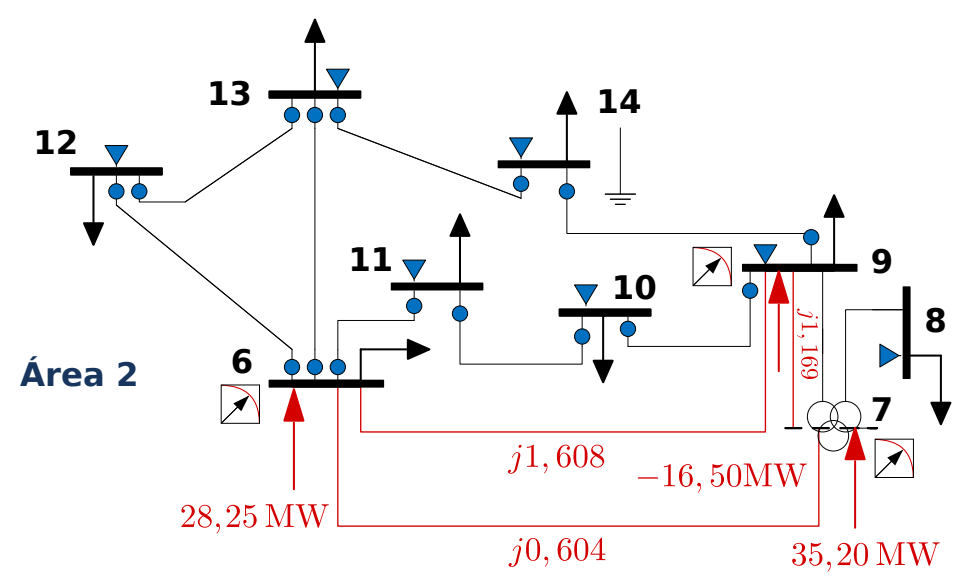

Eq. Ward Área 1

$\nabla$ - Med. Injeção $\quad-$ - Med. fluxo $\nabla$ - PMU

Figura 5.6 - Sistema IEEE-14: modelo reduzido da área 2

Tabela 5.5 - Comparação dos ângulos estimados (em graus), entre os métodos MQP linear convencional e MQP com equivalentes incorporados.

\begin{tabular}{c|r|r|r|r|r}
\hline \multirow{2}{*}{ Barra } & \multirow{2}{*}{$\hat{\theta}^{r e f}$} & \multicolumn{2}{|c|}{ Área 1} & \multicolumn{2}{c}{ Área 2} \\
\cline { 3 - 6 } & & $\hat{\theta}^{m q p}$ & $\hat{\theta}^{m q p+e q}$ & $\hat{\theta}^{m q p}$ & $\hat{\theta}^{m q p+e q}$ \\
\hline 1 & 16,394 & 16,394 & $\mathbf{1 6 , 3 9 4}$ & & \\
2 & 11,347 & 11,345 & $\mathbf{1 1 , 3 4 7}$ & & \\
3 & 3,383 & 3,378 & $\mathbf{3 , 3 8 4}$ & & \\
$4^{\star}$ & 5,734 & 5,721 & $\mathbf{5 , 7 3 4}$ & & \\
$5^{\star}$ & 7,236 & 7,217 & $\mathbf{7 , 2 3 6}$ & & \\
$6^{\star}$ & 1,106 & & & 1,112 & $\mathbf{1 , 1 1 1}$ \\
$7^{\star}$ & 2,236 & & & 2,192 & $\mathbf{2 , 2 3 7}$ \\
8 & 2,236 & & & 2,192 & $\mathbf{2 , 2 3 7}$ \\
$9^{\star}$ & 0,395 & & & 0,386 & $\mathbf{0 , 3 9 6}$ \\
10 & 0,091 & & & 0,085 & $\mathbf{0 , 0 9 1}$ \\
11 & 0,392 & & & 0,394 & $\mathbf{0 , 3 9 6}$ \\
12 & 0,000 & & & 0,012 & $\mathbf{0 , 0 0 7}$ \\
13 & $-0,158$ & & & $-0,148$ & $-\mathbf{0 , 1 5 1}$ \\
14 & $-1,142$ & & & $-1,142$ & $\mathbf{- 1 , 1 4 2}$ \\
\hline
\end{tabular}

Barras de fronteira estão marcadas com * 


\section{Capítulo 6}

\section{Testes e Validação dos Modelos}

Considerando os sistemas testes IEEE 30 e 118 barras, neste capítulo avalia-se o desempenho dos modelos propostos sob as óticas de precisão, custo computacional e robustez na presença de erros. Além do modelo de EEMA com equivalentes externos integrados, que será referido por Estimador de Estado com Equivalentes Externos incorporados (EEEQ), incluem-se nos testes os resultados dos métodos de dois níveis STLSE e ZHABSE, principais expoentes do contexto de EEH. Estratégias de EE distribuídas não são consideradas nas análises, na medida em que, $a$ priori, assume-se que levam a resultados ótimos.

O conjunto de medidas para cada cenário é gerado a partir dos resultados do Fluxo de Carga, que fornece os valores admitidos como verdadeiros, sendo então acrescidos de ruídos gaussianos com médias nulas e desvios-padrão proporcionais às grandezas medidas (ZHOU et al., 2006), da seguinte forma:

$$
z_{\text {med }}=z_{v}+\sigma_{z} X
$$

em que $z_{m e d}$ é o valor atribuído à medida; $z_{v}$ é o valor verdadeiro; $\sigma_{z}$ é o desvio padrão correspondente, definido conforme a Tab. (6.1); e $X \sim N(0,1)$ é uma variável aleatória com distribuição normal padrão. A componente relacionada ao fundo de escala do medidor, nesse caso, está omitida. O método numérico para teste de observabilidade desenvolvido em Monticelli e Wu (1985b) é utilizado para assegurar a geração de configurações observáveis para cada área.

Como visto no Capítulo 3, a referência angular de medidas fasoriais sincronizadas é determinada de acordo com o tempo UTC no instante que a medição é efetuada. Para a simplificação das análises, considera-se que o valor do ângulo do sincrofasor de tensão medido na barra de referência original do sistema sob estudo é $0^{\circ}$. Desta forma, a referência angular de áreas sincronizadas por PMUs será a mesma do estimador de referência. 
Tabela 6.1 - Desvios-padrão atribuídos para cada tipo de medida

\begin{tabular}{c|c|c|c|c|c}
\hline \hline \multicolumn{2}{c|}{ SCADA } & \multicolumn{4}{c}{ SMSF } \\
\hline$P_{k}^{m}, Q_{k}^{m}$ & $P_{k m}^{m}, Q_{k m}^{m}$ & $V_{k}^{m}$ & $\theta_{k}^{m}$ & $\left|I_{k m}^{m}\right|$ & $\alpha_{k m}^{m}$ \\
\hline $1 \%$ & $1 \%$ & $0,001 \%$ & $0,0067^{\circ}$ & $0,001 \%$ & $0,0067^{\circ}$ \\
\hline
\end{tabular}

Os resultados de estimação de estado são avaliados estatisticamente considerando, para cada método analisado, a média dos valores computados em um número predefinido de simulações $\left(n_{s}\right)$. O EEI, que considera todas as áreas e medidas como parte de um único sistema, é adotado como referência para todos os modelos. Assumindo que $\hat{\mathbf{x}}_{\mathbf{s}}^{\text {ref }}=\left[\begin{array}{ll}\theta_{\mathbf{s}}^{\text {ref }} & \mathbf{V}_{\mathbf{s}}^{\text {ref }{ }^{\prime}}\end{array}\right]^{\prime}$ é o estado obtido pelo modelo EEI na simulação $s$, a precisão de um método de EEMA poderá ser avaliada pelas métricas Erro absoluto médio de magnitude de tensão e Erro absoluto médio de ângulo de tensão, dadas por:

$$
\begin{gathered}
\overline{\mathbf{V}}_{e r r}=\frac{\sum_{s=1}^{n s}\left|\mathbf{V}_{\mathbf{s}}-\mathbf{V}_{\mathbf{s}}^{\text {ref }}\right|}{n_{s}} \\
\overline{\boldsymbol{\theta}}_{e r r}=\frac{\sum_{s=1}^{n s}\left|\boldsymbol{\theta}_{\mathbf{s}}-\boldsymbol{\theta}_{\mathbf{s}}^{\text {ref }}\right|}{n_{s}}
\end{gathered}
$$

em que $\hat{\mathbf{x}}_{\mathbf{s}}=\left[\begin{array}{ll}\boldsymbol{\theta}_{\mathbf{s}}{ }^{\prime} & \mathbf{V}_{\mathbf{s}}{ }^{\prime}\end{array}\right]^{\prime}$ representa o estado estimado pelo método sob análise.

De forma similar, considerando $\mathbf{r}(\hat{\mathbf{x}})$ e $\mathbf{h}_{\mathbf{s}}(\hat{\mathbf{x}})$ os vetores dos resíduos e das grandezas estimadas por um determinado método na simulação $s$, o Vetor dos valores médios estimados e o Vetor dos resíduos médios podem ser definidos como:

$$
\begin{gathered}
\overline{\mathbf{r}}(\hat{\mathbf{x}})=\frac{\sum_{s=1}^{n s} \mathbf{r}_{\mathbf{s}}(\hat{\mathbf{x}})}{n_{s}} \\
\overline{\mathbf{h}}(\hat{\mathbf{x}})=\frac{\sum_{s=1}^{n s} \mathbf{h}_{\mathbf{s}}(\hat{\mathbf{x}})}{n_{s}}
\end{gathered}
$$

Os modelos de EEMA considerados nesta tese foram implementados em um programa computacional codificado em linguagem $\mathrm{C}++$. Diante do elevado fluxo de dados e do complexo 
relacionamento dos distintos ramos de estudo envolvidos no problema, como Fluxo de Carga, Estimação de Estado tradicional e Equivalentes Externos, a filosofia de Programação Orientada a Objetos (POO) mostrou-se adequada para fundamentar as estruturas de dados desenvolvidas. Diferentemente da programação clássica modular, onde um determinado problema é representado em termos de chamadas de rotinas e estruturas de dados, a POO envolve a manipulação de conceitos, ou tipos de dados abstratos, conhecidos como classes. Conceitos semelhantes podem ser agrupados em uma mesma estrutura de dados, que podem então dar origem (ou herdarem) estruturas com mesmas características. Desta forma, é possível estabelecer um completo arcabouço do problema estudado em códigos reduzidos, mais claros e com maior potencial de reutilização e atualização.

Dentre os inúmeros pacotes computacionais de terceiros que foram integrados ao programa, podem ser citadas as bibliotecas do Boost $\mathrm{C}++$ (BOOST, 2013), utilizadas na geração de números aleatórios e na medição e tratamento de tempos computacionais, e as rotinas HSL-MA57 (HSL, 2013), que foram utilizadas para a solução de sistemas de equações lineares esparsos, sob distintas configurações. As diretivas do OpenMP (OPENMP, 2013), embutidas diretamente no compilador, tornaram possível o aumento do número de casos gerados e analisados e a redução dos tempos de simulação dos métodos. Os testes foram executados em um PC Intel(R) Core(TM) i7-3770 CPU @ $3.40 \mathrm{GHz}$ em um sistema operacional GNU-Linux, sob suporte da interface de desenvolvimento Eclipse (ECLIPSE, 2013).

\subsection{Sistema IEEE 30 barras}

Considerando o sistema IEEE-30 particionado em duas áreas, conforme a Fig. (6.1), esta análise introdutória visa avaliar a precisão das estimativas obtidas nos modelos propostos em relação aos modelos STLSE e EEI (completo). O plano de medição utilizado considera medidas de fluxo de potência ativa e reativa em todos os ramos e medidas de injeção de potência nas barras de fronteira. Unidades de medição fasorial, igualmente alocadas nas fronteiras, disponibilizam sincrofasores de tensão e de corrente, conforme o modelo mostrado na Fig. (3.4). Os tipos de barras definidas no particionamento utilizado estão listadas na Tab. (6.2).

O primeiro nível de estimação do STLSE corresponde a estimadores convencionais por MQP, em que redes e medidas externas são desconsideradas. Injeções em barras de fronteira também são ignoradas, como referido na Seção (2.2.2). A etapa de coordenação procede da forma já discutida, onde medidas de fluxo em ramos de interconexão são utilizadas para refinar estimativas de fronteira e determinar o vetor de coordenação u. As barras de geração 11 (área 1) e 13 (área 2) fornecem as referências angulares locais para o método STLSE, com valores arbitrados em $0^{\circ}$. Esta última barra é também a referência angular do modelo integrado. 


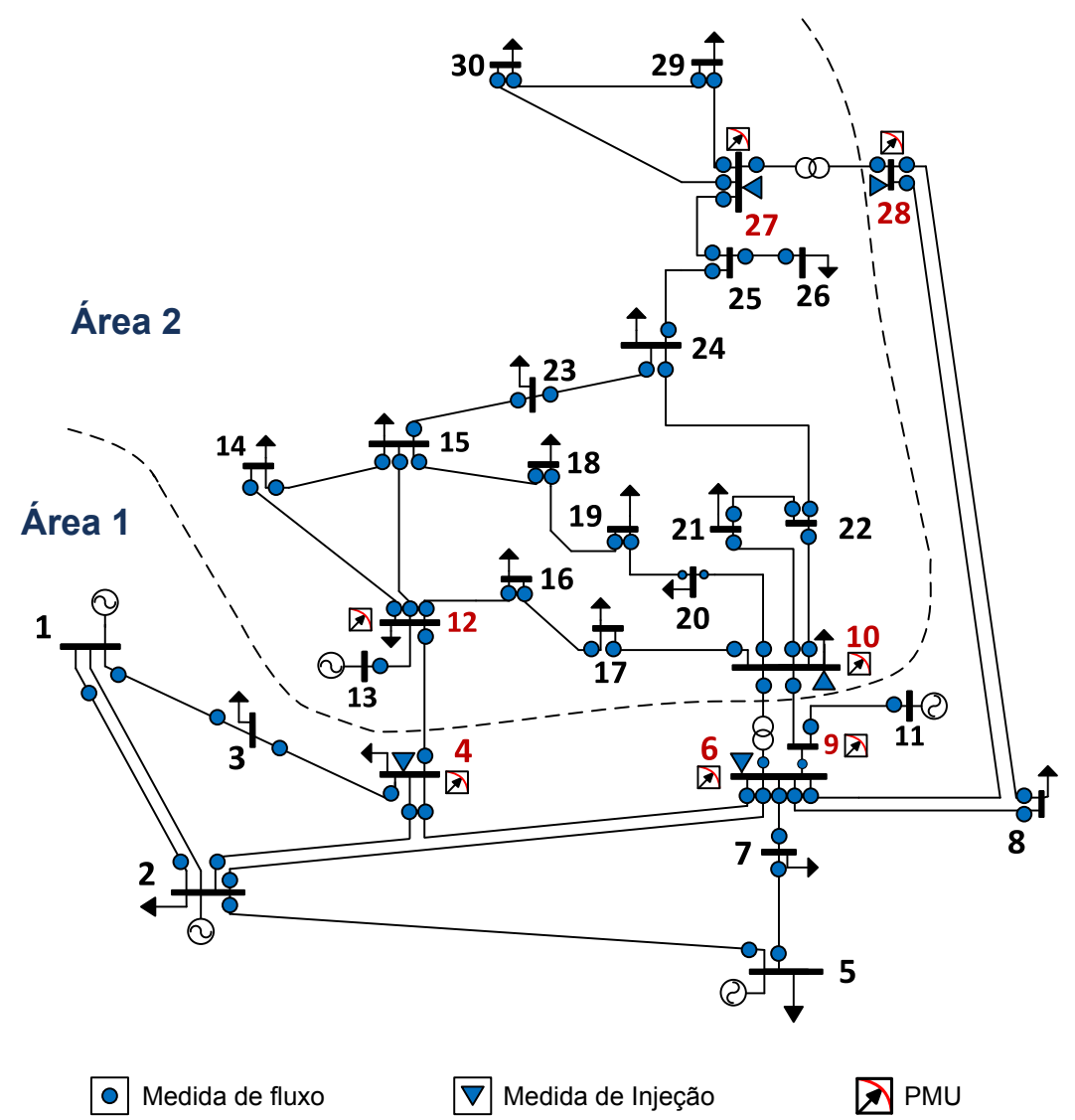

Figura 6.1 - Sistema IEEE-30: configuração das subáreas

Tabela 6.2 - Sistema IEEE-30: partição em duas áreas

\begin{tabular}{c|c|c|c|c}
\hline \hline \multirow{2}{*}{ Área } & \multirow{2}{*}{$\begin{array}{c}\text { Barra de } \\
\text { referência }\end{array}$} & \multicolumn{3}{|c}{ Quantidade de Barras } \\
\cline { 3 - 5 } & & Internas & Fronteira & Primeira vizinhança \\
\hline 1 & 11 & 7 & $4(4,6,9,28)$ & $3(10,12,27)$ \\
\hline 2 & 13 & 16 & $3(10,12,27)$ & $4(4,6,9,28)$ \\
\hline
\end{tabular}


No método EEEQ, considera-se que as medidas de ângulo de tensão nas barras de fronteira 4 e 27, diretamente monitoradas por PMUs, fornecem as referências internas para as respectivas áreas. Para o cálculo de EQEs, assume-se também que fronteiras são expandidas às barras de primeira vizinhança. Este procedimento, necessário para a inclusão no modelo de medidas em regiões de borda (injeções em barras de fronteira e fluxos em ramos de interconexão), é descrito de forma sucinta a seguir.

\subsubsection{Configuração do EEEQ com retenção de barras de primeira vizinhança}

Como discutido na Seção (4.1), circuitos equivalentes do EQE Ward são obtidos por eliminação gaussiana na matriz Admitância Nodal do sistema completo. Para a área 1 do sistema em questão, a estrutura desta matriz está ilustrada na Fig. (6.2), reorganizada nos blocos fronteira $(f)$, primeira vizinhança $(n)$ e externo $(e)$. Elementos não nulos estão assinalados com " $\times$ ". Barras internas não são mostradas, na medida em que não estão diretamente envolvidas no processo de redução.

Procedendo-se à eliminação de Gauss no conjunto das barras externas, obtêm-se a matriz representada na Fig. (6.3). A rede equivalente de Ward para a área 1 está grafada em negrito, associada às admitâncias equivalentes $y_{10-12}, y_{10-27}$ e $y_{12-27}$. Verifica-se que, neste caso, ramos de interconexão originais são mantidos, juntamente com as medidas associadas. Este procedimento equivale, em outras palavras, à retenção de barras de primeira vizinhança na eliminação de Gauss. Aplicando-se o mesmo raciocínio para a área 2, as admitâncias equivalentes $y_{4-6}, y_{6-28}$ são obtidas.

Após a determinação dos circuitos equivalentes, injeções de potência em barras de primeira vizinhança podem ser obtidas a partir das Equações (4.15) e (4.16). Como afirmado anteriormente, estas injeções são dependentes dos sincrofasores instantâneos de tensão e corrente medidos por PMUs. O aspecto geral do sistema IEEE-30 com circuitos equivalentes incorporados é representado na Fig. (6.4), onde verifica-se que as conexões entre sistemas foram eliminadas, permitindo ao problema de EEMA ser solucionado de forma descentralizada.

\subsubsection{Estado Estimado}

O desempenho do método proposto é analisado, a seguir, com base na média do estado estimado em 1000 simulações $\left(n_{s}=1000\right)$. Os valores das medidas nas $n_{s}$ execuções são gerados adicionando-se ruídos aleatórios aos resultados do fluxo de carga referente ao caso base do sistema, conforme a Eq. (6.1). Neste estudo preliminar, somente os resultados da Área 2 serão considerados. As nomenclaturas EEEQ e EEEQp serão utilizadas nesta seção, respectivamente, 


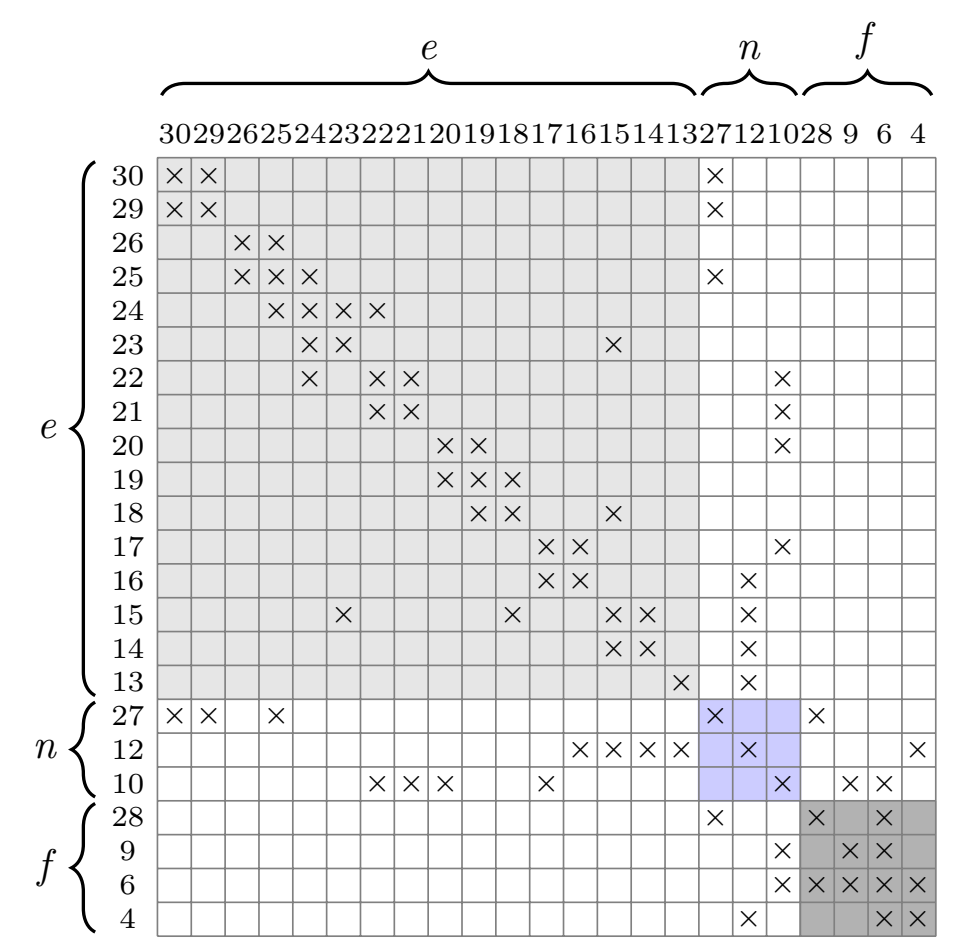

Figura 6.2 - Sistema IEEE-30: matriz admitância nodal completa vista pela área 1

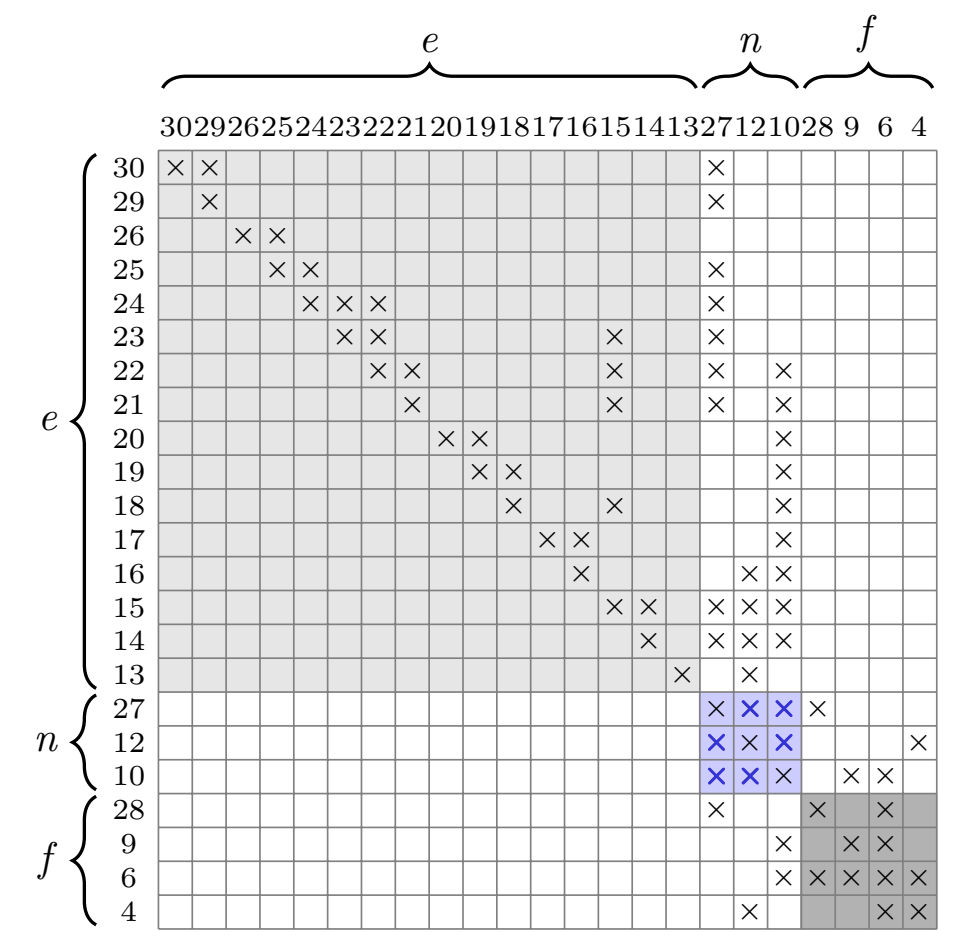

Figura 6.3 - Sistema IEEE-30: matriz admitância nodal reduzida, área 1 


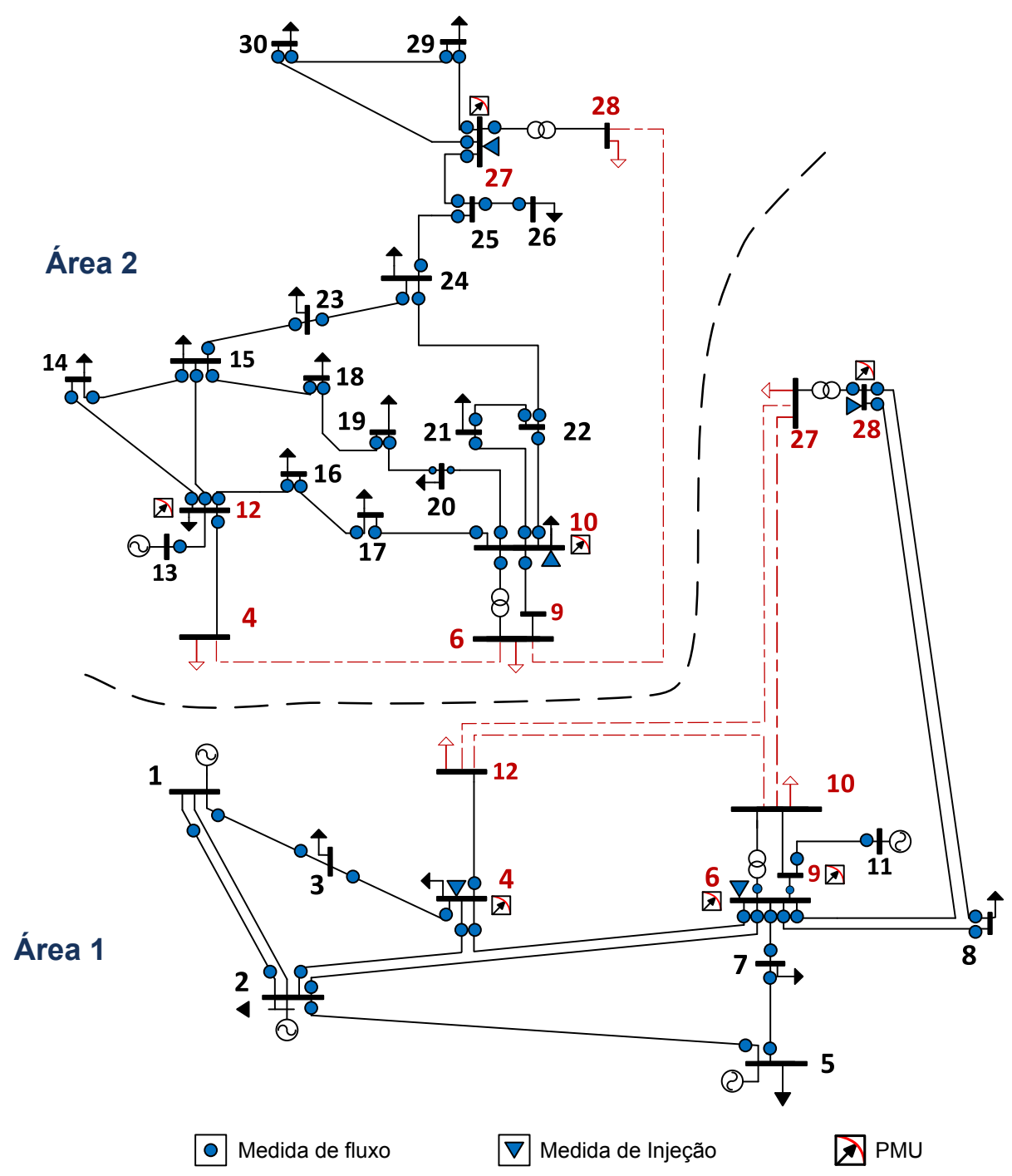

Figura 6.4 - Sistema IEEE-30: rede completa com equivalentes incorporados

para referir-se à inclusão de EQEs como restrições de igualdade e como medidas convencionais a serem ponderadas. Assume-se que a topologia do sistema não é alterada ao longo dos cenários gerados, sendo o ajuste de fronteira efetuado com base nos valores medidos pelas PMUs. Nesta última estratégia, três pesos são considerados: $10^{-3}, 10^{-4}$ e $10^{-5}$. Considera-se inicialmente que os EQEs são calculados pelo método Ward convencional.

Os erros médios de magnitude e ângulo de tensão em relação ao estado de referência (método EEI), computados para os métodos EEEQ, EEEQp e STLSE, na área 2, são mostrados nas Figuras (6.5) e (6.6). Nota-se primeiramente que os desvios em relação à referência são muito baixos em todas as metodologias, fato geralmente observado no problema de EE multiárea. Pondera-se 


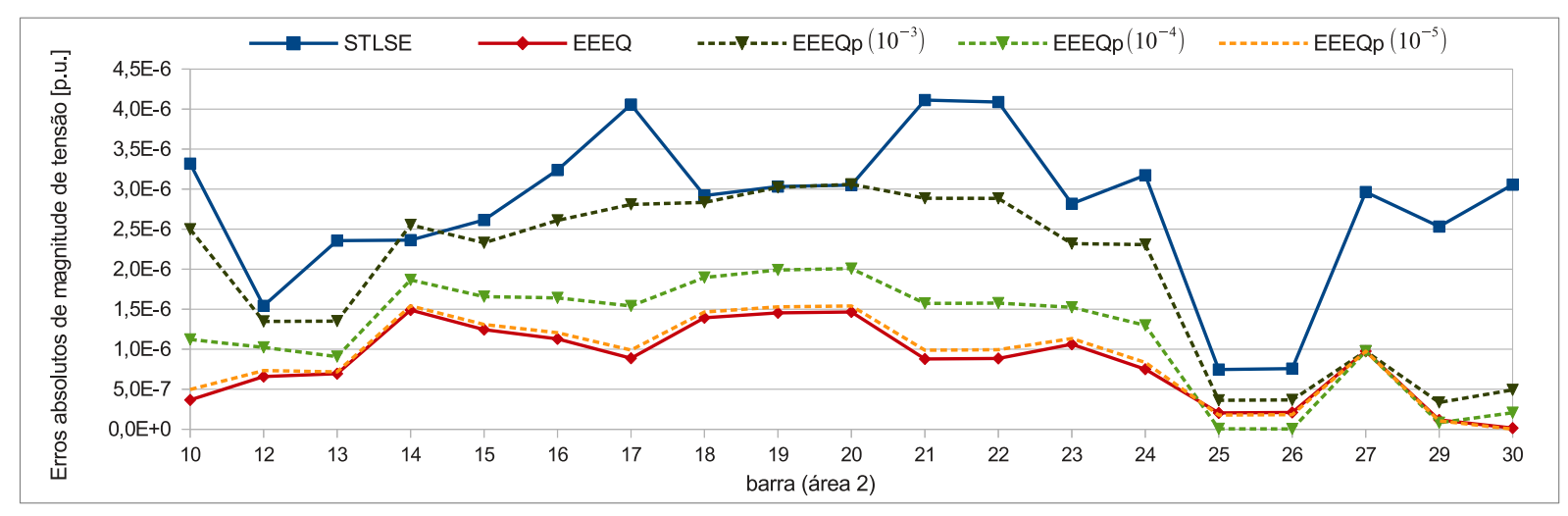

Figura 6.5 - Erros absolutos de magnitude de tensão na área 2 em relação ao EEI: modelos STLSE, EEEQ (método proposto com injeções equivalentes incorporadas como restrições de igualdade) e EEEQp (injeções incorporadas como pseudomedidas, pesos $10^{-3}, 10^{-4}$ e $10^{-5}$ )

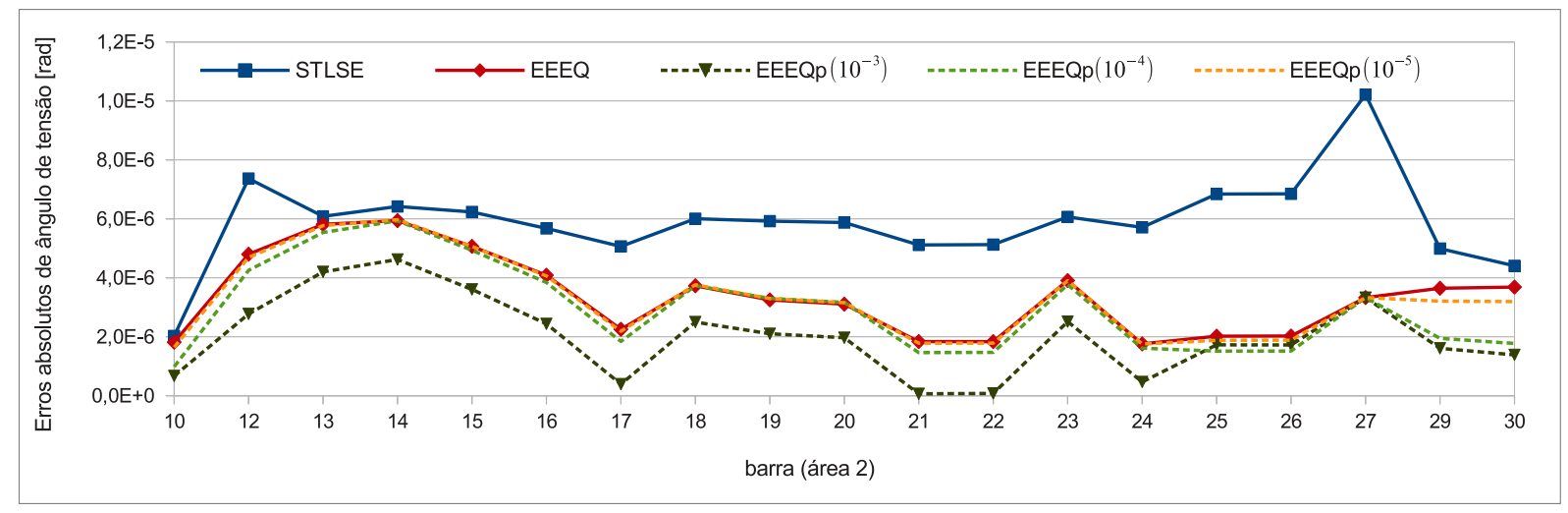

Figura 6.6 - Erros absolutos de ângulo de tensão na área 2 em relação ao EEI: modelos STLSE, EEEQ e EEEQp

que erros em sistemas interconectados tendem a se concentrar em áreas de fronteira e zonas críticas, como barras vizinhas a linhas curtas (VAN CUTSEM; HORWARD; RIBBENS-PAVELLA, 1981). No entanto, constata-se que estimativas precisas foram obtidas pelo método EEEQ mesmo sem a execução de uma etapa de coordenação, como no método STLSE.

Em segundo lugar, verifica-se que as estimativas dos modelos EEEQ e EEEQp tendem a se aproximar à medida que pesos maiores são atribuídos às injeções equivalentes. Resultados similares foram observados, nesta simulação, para desvios-padrão menores que $10^{-5}$. Todavia, como já mencionado, o uso de medidas com variâncias distintas pode ocasionar problemas de mal condicionamento no processo iterativo. A modelagem por restrições de igualdade pode ser utilizada para contornar esses problemas e, ao mesmo tempo, incrementar a precisão do método. 


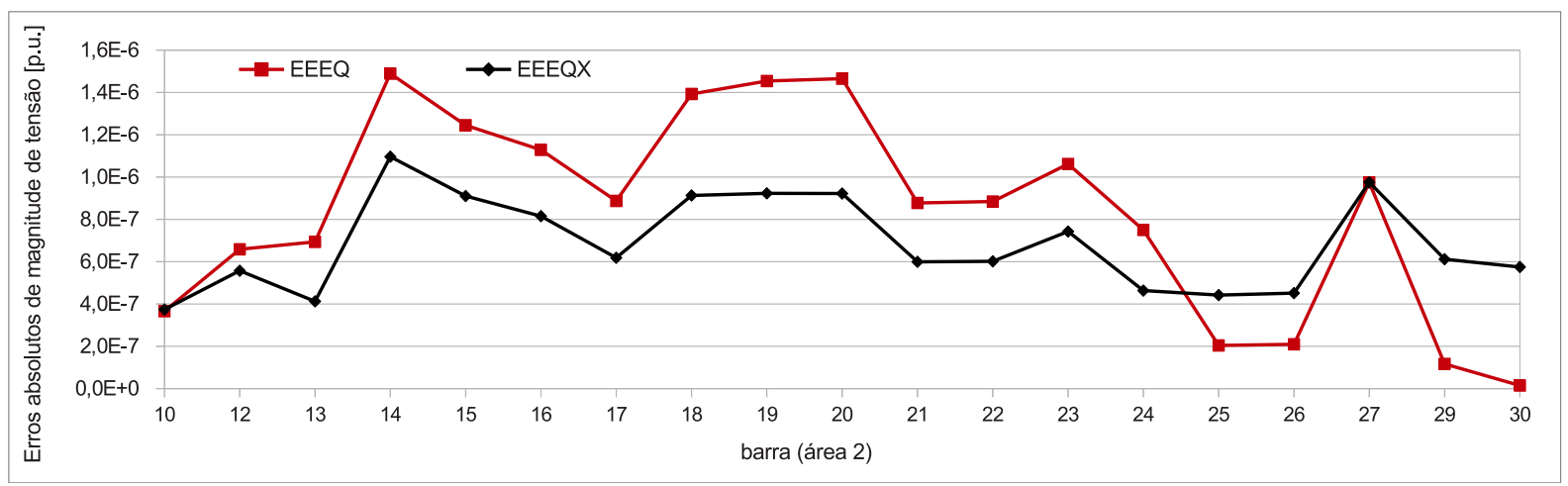

Figura 6.7 - Erros absolutos de magnitude de tensão (área 2) para o modelo com restrições de igualdade utilizando os métodos Ward clássico (EEEQ) e Ward Estendido (EEEQx).

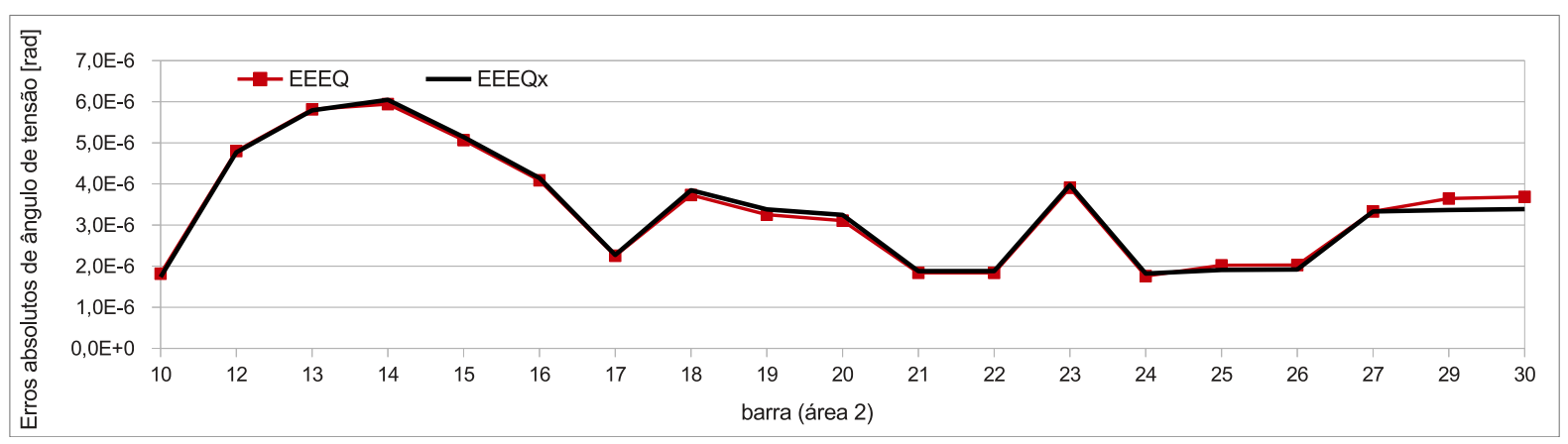

Figura 6.8 - Erros absolutos de ângulo de tensão (área 2) para o modelo com restrições de igualdade utilizando os métodos Ward clássico (EEEQ) e Ward Estendido (EEEQx).

\section{Resultados considerando o modelo Ward Estendido}

Considerando o mesmo cenário anterior, apresentam-se nas Figuras (6.7) e (6.8) os erros de magnitude e ângulo de tensão quando EQEs são computados utilizando os métodos Ward Clássico (abordagem do teste anterior) e Ward Estendido (estratégia referida no gráfico por EEEQx). Em ambos os modelos é utilizada a formulação por restrições de igualdade. É possível constatar uma leve melhora nas estimativas, sobretudo nos valores de magnitude de tensão, que pode ser compreendida como um efeito direto dos suportes reativos adicionais em fronteiras. As vantagens do modelo estendido em relação ao método Ward convencional podem chegar a ser consideráveis, especialmente diante de contingências (MONTICELLI et al., 1979).

Diante dos resultados iniciais obtidos, a configuração padrão adotada para o método EEEQ nos próximos testes contemplará o cálculo de EQEs pelo método Ward Estendido e a integração de informações em tempo real como restrições de igualdade. 


\subsection{Sistema IEEE 118 barras - partição em 3 Áreas}

Este teste visa estender as análises anteriores para o sistema IEEE 118 barras, sendo incluídos também os resultados do método ZHABSE. A estratégia de partição adotada, também considerada em Korres (2011), está ilustrada na Fig. (6.9). Informações das áreas relacionadas, como o número de barras $\left(n_{b}\right)$ e os nós pertencentes a cada uma delas estão descritas na Tab. (6.3)

Três casos com distintos planos de medição são analisados, conforme descrito na Tab. (6.4). O primeiro consiste, à semelhança do estudo anterior, em fluxos de potência em todas as linhas, injeções de potência e medidas fasoriais sincronizadas em barras de fronteira. O segundo apresenta uma redundância média ${ }^{1}$ de 1,54 por área, onde são consideradas medidas de fluxo em somente um dos sentidos das linhas, além de injeções de potência em barras de fronteira. Já o terceiro caso considera fluxos em todas as linhas e injeções de potência em todas as barras, ocasionando uma redundância média de 3,64. O número de variáveis de estado $(N)$ de cada área é determinado considerando o número total de barras $\left(n_{b}\right)$ e o número de barras de primeira vizinhança $\left(n_{b n}\right)$, ou seja, $N=2\left(n_{b}+n_{b n}\right)-1$.

Pseudomedidas de magnitude de tensão (geradas a partir do fluxo de carga) são alocadas às barras 19, 65 e 69, que fornecem as referências angulares $\left(0^{\circ}\right)$ para os métodos STLSE e ZHABSE. Para estas informações, desvios-padrão de $\sigma=10^{-6}$ p.u. são adotados. No método EEEQ, os ângulos de tensão medidos pelas PMUs nas mesmas barras (que também são barras de fronteira) fornecem as próprias referências internas. Similarmente, assume-se que não há mudanças topológicas após a construção dos EQEs, como considerado no último teste.

Os valores médios dos erros de magnitude e ângulo de tensão, computados para cada modelo em $n s=5000$ simulações, no caso 1, estão representados nas Figuras (6.10) e (6.11). Verifica-se que a consideração de medidas de injeção de potência em estimadores de dois níveis, como no método ZHABSE, leva à obtenção de melhores estimativas que o modelo padrão STLSE. No entanto, é possível ponderar que, mesmo considerando uma etapa adicional de estimação, erros significantes de magnitude e ângulo de tensão ainda estão presentes, sobretudo em zonas de fronteira, como pode ser observado na Fig. (6.10) (verificar barras de fronteira 47,65, 69 e outras em negrito).

Nas Fig. (6.12) e (6.13), observa-se que a ocorrência de "picos" de erros de estimativas nas barras de fronteira permanece nos métodos de dois níveis mesmo diante de planos de medição distintos. Na pior situação (caso 2), aumenta-se a quantidade de picos, como é possível notar no gráfico (6.12), barras 36 e 40. No melhor nível de redundância (caso 3), o método EEEQ

\footnotetext{
${ }^{1} \mathrm{~A}$ redundância média será dada pela média dos valores de redundância observados em cada área.
} 


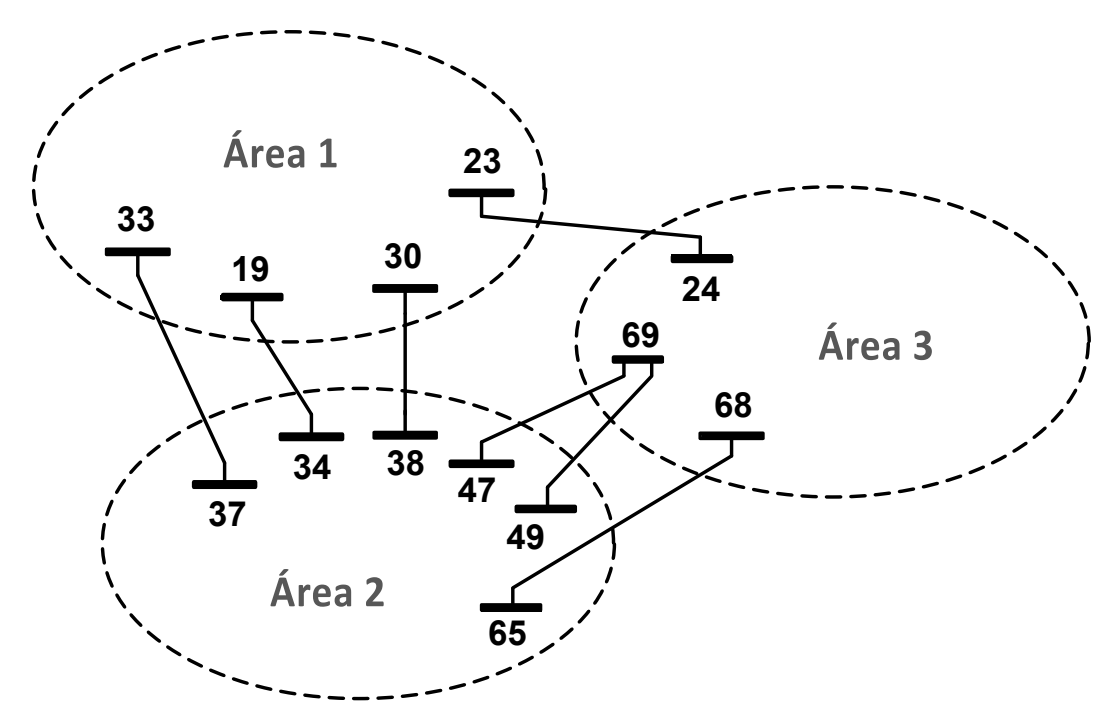

Figura 6.9 - Sistema IEEE-118: configuração em três subáreas

Tabela 6.3 - Sistema IEEE-118: partição em três áreas.

\begin{tabular}{c|c|c}
\hline \hline Área & $n_{b}$ & Barras \\
\hline 1 & 36 & $1-23,25-33,113-115,117$ \\
\hline 2 & 34 & $34-67$ \\
\hline 3 & 48 & $24,68-112,116,118$ \\
\hline
\end{tabular}

Tabela 6.4 - Sistemas IEEE-118 (3 áreas): níveis de redundância analisados $(\eta=m / N)$

\begin{tabular}{c|c|c|c|c|c|c|c}
\hline \hline \multirow{2}{*}{ Área } & \multirow{2}{*}{ Variáveis de estado } & \multicolumn{2}{|c|}{ Caso 1} & \multicolumn{2}{c|}{ Caso 2} & \multicolumn{2}{c}{ Caso 3 } \\
\cline { 3 - 8 } & & $m$ & $\eta$ & $m$ & $\eta$ & $m$ & $\eta$ \\
\hline 1 & 79 & 214 & 2,70 & 116 & 1,46 & 278 & 3,51 \\
\hline 2 & 77 & 234 & 3,03 & 130 & 1,68 & 290 & 3,76 \\
\hline 3 & 103 & 300 & 2,91 & 158 & 1,53 & 390 & 3,78 \\
\hline
\end{tabular}




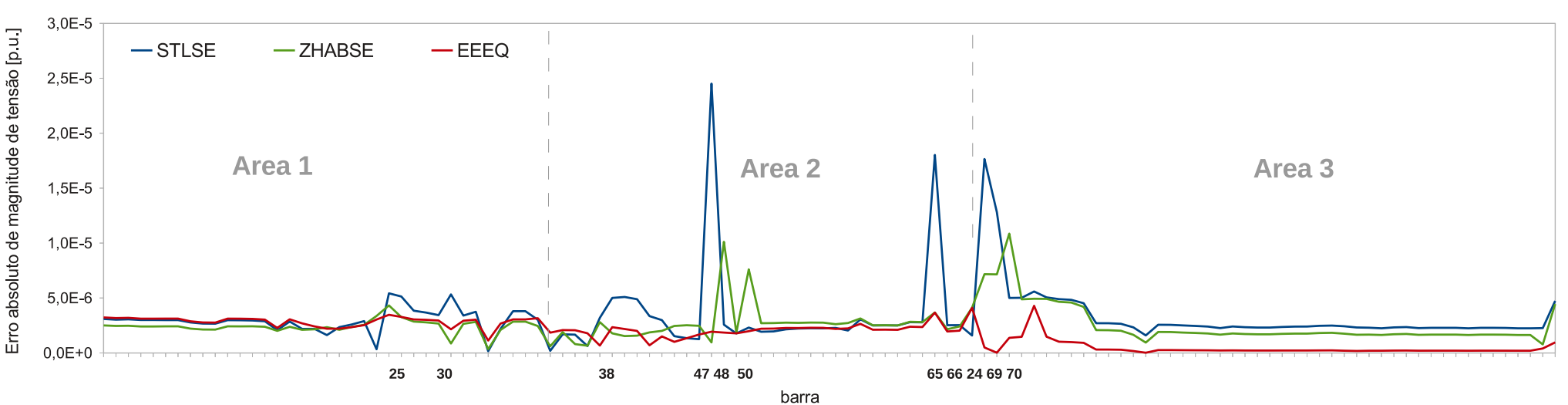

Figura 6.10 - Sistema IEEE-118 (3 áreas): erros absolutos de magnitude de tensão para o caso 1

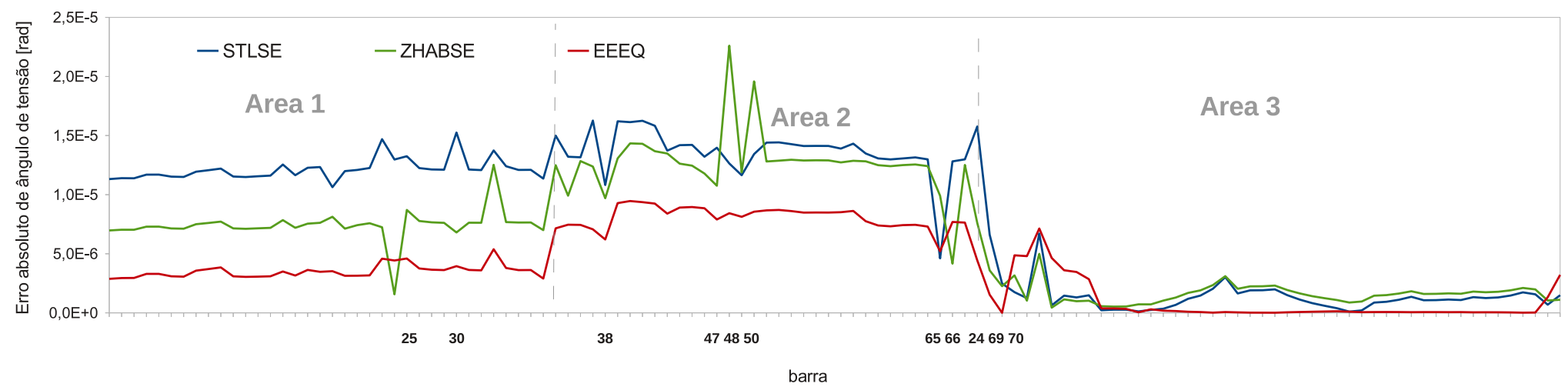

Figura 6.11 - Sistema IEEE-118 (3 áreas): erros absolutos de ângulo de tensão para o caso 1 


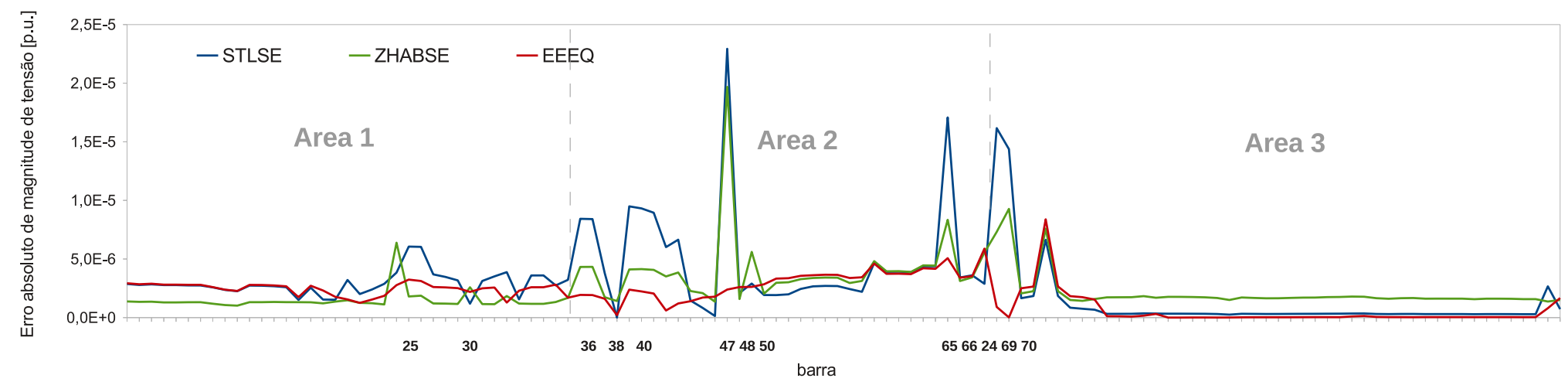

Figura 6.12 - Sistema IEEE-118 (3 áreas): erros absolutos de magnitude de tensão para o caso 2 (redundância reduzida)

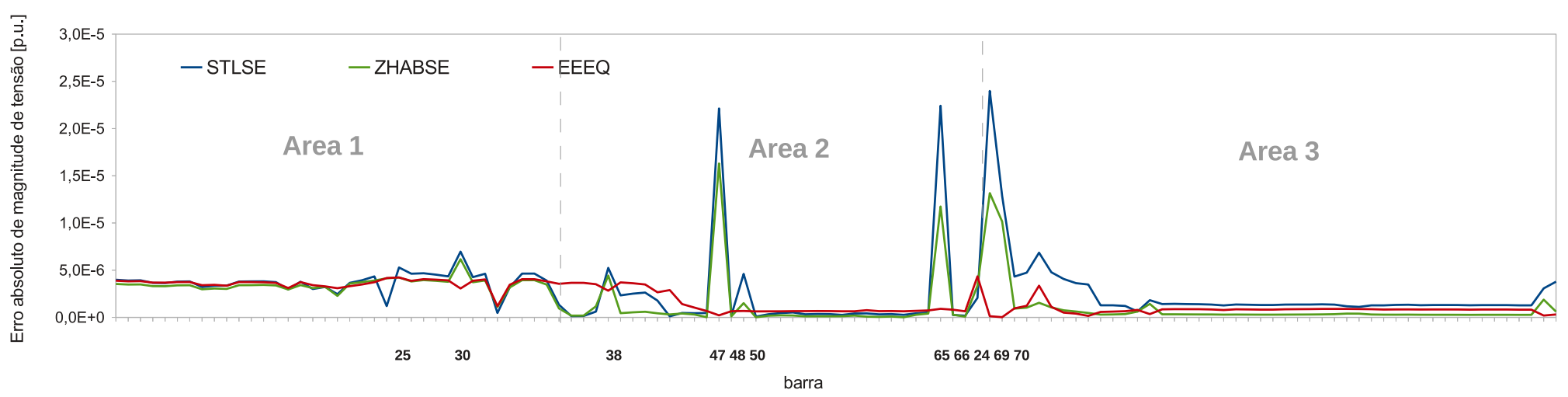

Figura 6.13 - Sistema IEEE-118 (3 áreas): erros absolutos de magnitude de tensão para o caso 3 (redundância elevada) 
Tabela 6.5 - Sistema IEEE-118 (3 áreas): índices $J(x)$ médios observados no caso 3 (redundância elevada)

\begin{tabular}{c|c|c|c|c}
\hline \hline & STLSE & ZHABSE & EEEQ & EEI \\
\hline Área 1 & 49,43 & 46,14 & 31,8 & - \\
\hline Área 2 & 364,66 & 145,8 & 33,89 & - \\
\hline Área 3 & 11776,3 & 1206,27 & 62,287 & - \\
\hline Geral & 12190,3 & 1398,21 & 127,97 & $\mathbf{1 2 5 , 9 9}$ \\
\hline
\end{tabular}

ocasiona um índice $J(x)$ médio de 127,98, contra 125,99 no método EEI, conforme mostrado na Tab. (6.5). Nesta mesma situação, os modelos STLSE e ZHABSE convergem, respectivamente, para os valores médios 12190,3 e 1306,27. Nota-se que os índices $J(x)$ associados às áreas 2 e 3 não são devidamente minimizados nestes dois últimos métodos, sobretudo no modelo STLSE.

A análise dos resíduos médios de certas medidas das áreas 2 e 3, apresentados na Tab. (6.6), pode ser utilizada para a compreensão deste problema. Listam-se na tabela os valores medidos $(z)$, estimados $(h(\hat{x}))$ e os resíduos associados a cada medida $(h(\hat{x}))$, para o caso 3 . O índice $J_{i}(x)$, que representa a contribuição ao resíduo final de cada medida, também é incluído. Tomando-se o método STLSE como exemplo, é possível constatar um desvio de 0,008 p.u. em relação aos valores medidos e estimados para $Q_{68-116}$ (área 3). O mesmo comportamento pode ser observado para os resíduos de $Q_{116}$ (área 3), tanto para este último método quanto para o ZHABSE. Resíduos elevados são detectados também em todas as medidas listadas da área 2, no modelo STLSE. Nota-se que os maiores resíduos estão localizados em regiões de fronteira, como por exemplo, na vizinhança da barra 116.

É possível concluir que a reestimação do estado de fronteira, em métodos de dois níveis, vem a alterar significativamente os resíduos locais. Este efeito não é observado no método proposto, indicando que injeções equivalentes em tempo real atuam como medidas compensatórias no problema de EEMA. Tal propriedade não é alcançada com a reestimação de certas variáveis de estado próximas às barras de fronteira, baseando-se em índices de sensibilidades, proposta sugerida em Jiang, Vittal e Heydt (2007).

\subsection{Sistema IEEE 118 barras - partição em 9 Áreas}

Este teste visa avaliar o custo computacional dos modelos anteriores, considerando o sistema IEEE-118 decomposto em 9 áreas, conforme o diagrama da Fig. (6.14). Esta estratégia de partição é também considerada em Zhao e Abur (2005). 
Tabela 6.6 - Sistema IEEE-118 (3 áreas): valores estimados e resíduos para o caso 3 (redundância elevada)

\begin{tabular}{|c|c|c|c|c|c|c|c|c|c|c|}
\hline \multirow{2}{*}{ Medida } & \multirow{2}{*}{$\begin{array}{l}\text { Valor em } \\
\text { p.u. }(z)\end{array}$} & \multicolumn{3}{|c|}{ STLSE } & \multicolumn{3}{|c|}{ ZHABSE } & \multicolumn{3}{|c|}{ EEEQ } \\
\hline & & $h(\hat{x})$ & $r(\hat{x})$ & $J_{i}(x)$ & $h(\hat{x})$ & $r(\hat{x})$ & $J_{i}(x)$ & $h(\hat{x})$ & $r(\hat{x})$ & $J_{i}(x)$ \\
\hline \multicolumn{11}{|l|}{ Área 2} \\
\hline$Q_{64-65}$ & $-0,27338$ & $-0,27412$ & 0,00074 & 65,85 & $-0,27373$ & 0,00035 & 6,74 & $-0,27339$ & 0,00001 & 0,06 \\
\hline$Q_{65-66}$ & 0,45172 & 0,45270 & 0,00098 & 49,26 & 0,45211 & 0,00039 & 5,06 & 0,45173 & 0,00001 & 0,07 \\
\hline$Q_{65-64}$ & $-0,08473$ & $-0,08360$ & 0,00113 & 66,50 & $-0,08430$ & 0,00043 & 6,75 & $-0,08471$ & 0,00002 & 0,04 \\
\hline$Q_{66-65}$ & $-0,40472$ & $-0,40535$ & 0,00062 & 46,26 & $-0,40503$ & 0,00031 & 4,74 & $-0,40471$ & 0,00001 & 0,06 \\
\hline$Q_{66}$ & $-0,47195$ & $-0,47268$ & 0,00073 & 48,72 & $-0,47223$ & 0,00028 & 7,38 & $-0,47194$ & 0,00001 & 0,03 \\
\hline \multicolumn{11}{|l|}{ Área 3} \\
\hline$P_{68-116}$ & $-0,65955$ & $-0,66023$ & 0,00069 & 32,91 & $-0,66014$ & 0,00059 & 76,93 & $-0,65950$ & 0,00005 & 0,18 \\
\hline$P_{116-68}$ & 0,65979 & 0,66096 & 0,00117 & 33,01 & 0,66068 & 0,00089 & 76,67 & 0,66002 & 0,00023 & 0,19 \\
\hline$P_{116}$ & 0,66024 & 0,66096 & 0,00072 & 33,08 & 0,66068 & 0,00044 & 76,90 & 0,66002 & 0,00022 & 0,18 \\
\hline$Q_{68-69}$ & 0,29337 & 0,29383 & 0,00046 & 32,68 & 0,29341 & 0,00004 & 1,35 & 0,29334 & 0,00003 & 0,07 \\
\hline$Q_{68-81}$ & 2,26461 & 2,26682 & 0,00221 & 157,67 & 2,26577 & 0,00116 & 14,55 & 2,26508 & 0,00047 & 2,03 \\
\hline$Q_{68-116}$ & $-1,32818$ & $-1,31977$ & 0,00840 & 3707,63 & $-0,27632$ & 0,00008 & 1,29 & $-1,32801$ & 0,00017 & 0,59 \\
\hline$Q_{69-68}$ & $-0,27624$ & $-0,27648$ & 0,00023 & 31,07 & $-1,32476$ & 0,00342 & 290,92 & $-0,27626$ & 0,00002 & 0,06 \\
\hline$Q_{81-68}$ & $-2,97582$ & $-2,97736$ & 0,00154 & 137,06 & $-2,97683$ & 0,00101 & 14,54 & $-2,97625$ & 0,00043 & 3,69 \\
\hline$Q_{116-68}$ & 1,15052 & 1,14534 & 0,00519 & 3776,97 & 1,14791 & 0,00262 & 295,80 & 1,15092 & 0,00040 & 0,49 \\
\hline$Q_{116}$ & 1,15096 & 1,14534 & 0,00563 & 3779,23 & 1,14791 & 0,00305 & 296,95 & 1,15092 & 0,00004 & 0,18 \\
\hline
\end{tabular}




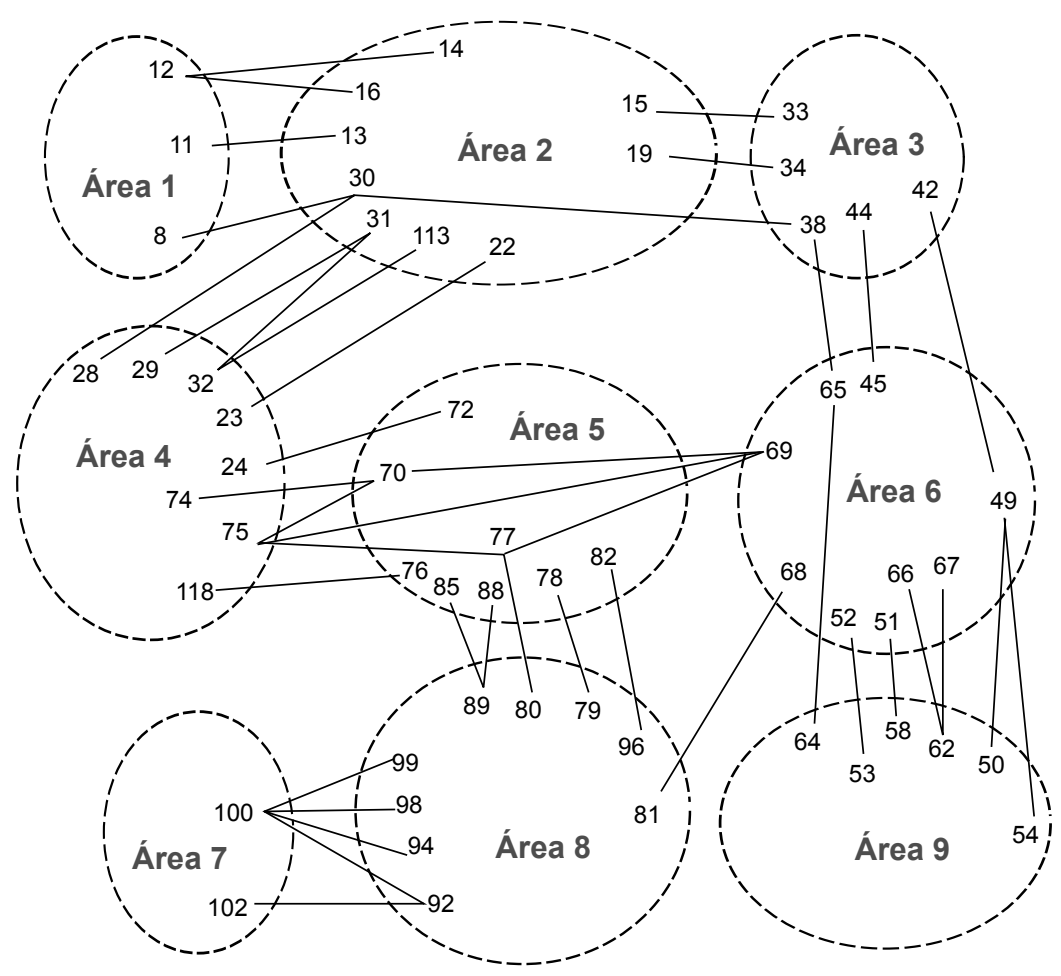

Figura 6.14 - Sistema IEEE-118: configuração em nove subáreas

Na Tabela (6.7) mostram-se os níveis de redundância por área, associados ao plano de medição utilizado, que segue o nível básico de redundância utilizada nos testes anteriores (fluxos em todas as linhas e injeções nas barras de fronteira). Nesta configuração, pode-se constatar que a área 4 perderá a observabilidade na ausência de certas medidas de injeção em barras de fronteira. Logo, o método STLSE, onde tais medidas são obrigatoriamente descartadas, não será considerado neste estudo.

O desempenho dos métodos EEEQ, ZHABSE e EEI (referência) é avaliado após a execução de $n s=5000$ simulações. Na Tabela (6.8) são mostrados os tempos computacionais médios obtidos, sendo destacadas as principais rotinas de cada modelo, como WardRT (Cálculo de EQEs em tempo real), $S E$ (Estimadores de Estado Locais), DelMC ${ }^{2}$ (Tempo de transmissão de medidas ao agente de coordenação) e Central (Estimador da área central). Os valores mostrados representam os somatórios dos totais observados em cada área. O símbolo "-" é utilizado quando uma função não se aplica a um determinado modelo.

\footnotetext{
${ }^{2}$ Representa somente o tempo de cópia das variáveis relevantes das estruturas computacionais locais para a estrutura de centralização. Não são considerados delays de comunicação.
} 
Tabela 6.7 - Sistema IEEE-118: partição em nove áreas.

\begin{tabular}{c|c|c|c|c|c}
\hline Área & $n_{b}$ & Barras & $\begin{array}{c}\text { Variáveis } \\
\text { de Estado }\end{array}$ & $\begin{array}{c}\text { Medidas } \\
(m)\end{array}$ & $\begin{array}{c}\text { Redundância } \\
(m / N)\end{array}$ \\
\hline 1 & 13 & $1-12,117$ & 33 & 80 & 2,42 \\
\hline 2 & 13 & $13-22,30,31,113$ & 45 & 94 & 2,08 \\
\hline 3 & 12 & $33-44$ & 35 & 80 & 2,28 \\
\hline 4 & 13 & $23-29,32,74,75,114,115,118$ & 43 & 92 & 2,14 \\
\hline 5 & 14 & $70-73,76-78,82-88$ & 45 & 94 & 2,08 \\
\hline 6 & 13 & $45-49,51,52,65-69,116$ & 51 & 110 & 2,15 \\
\hline 7 & 13 & $100-112$ & 33 & 84 & 2,54 \\
\hline 8 & 14 & $79-81,89-99$ & 43 & 110 & 2,55 \\
\hline 9 & 13 & $50,53-64$ & 37 & 100 & 2,70 \\
\hline
\end{tabular}

Tabela 6.8 - Sistema IEEE-118 (9 áreas): tempos computacionais médios (s) em 5000 simulações.

\begin{tabular}{c|c|c|c|c|l}
\hline \multirow{2}{*}{ Método } & \multicolumn{4}{|c|}{ Função } & \multirow{2}{*}{ Tempo total(s) } \\
\cline { 2 - 5 } & WardRT & SE & DelMC & Central & \\
\hline EEEQ & 0,006 & 0,143 & - & - & $\mathbf{0 , 1 4 9}(3,8 \%)$ \\
ZHABSE & - & 0,131 & 0,001 & 2,385 & $\mathbf{2 , 5 1 7}(59,8 \%)$ \\
EEI & - & 4,209 & - & - & $\mathbf{4 , 2 0 9}(100,0 \%)$ \\
\hline
\end{tabular}

Constata-se que, para um mesmo plano de medição, o método proposto é executado em apenas $3,8 \%$ do tempo computacional do modelo completo. O custo associado ao cálculo de injeções equivalentes em tempo real é desprezível. Os resultados mostram também que o tempo total de execução dos estimadores locais, para o método ZHABSE, é levemente inferior ao EEEQ, e que o tempo associado ao envio de dados ao estimador de coordenação é muito baixo (sem considerar os atrasos associados aos sistemas de comunicação).

Entretanto, pode-se notar também que o estimador central, no método ZHABSE, é executado em quase $56 \%$ do tempo de execução do modelo de referência, fato decorrente principalmente das elevadas dimensões da área de interconexão, que envolve não somente barras de fronteira, mas os nós vizinhos a elas. Nesta simulação, a área de coordenação compõe 103 barras (quase todo o 
sistema interconectado), ou seja, 214 variáveis de estado $(2 \times 103+8)$, que estão relacionadas a 630 medidas, dentre fluxos em interconexões, injeções de potência em fronteiras, e pseudomedidas de ângulo e magnitude de tensão nas barras envolvidas. A inexistência de uma etapa centralizadora é uma das maiores vantagens do método proposto em relação aos estimadores de dois níveis.

Por fim, nas Figuras (6.15) e (6.16) pode-se verificar a precisão superior alcançada pelo modelo EEEQ mesmo quando medidas de magnitude de tensão são incluídas no método ZHABSE (abordagem referida nos gráficos como ZHABSEv). Nota-se que estas medidas adicionais permitem a melhora das estimativas em barras de fronteira e vizinhanças, todavia erros consideráveis em relação ao EEI não são eliminados, sobretudo em barras de fronteira (observar os erros de magnitude de tensão nas barras 93, 95, 97 e de ângulo nas barras 23, 25, 29, 118). A compensação de fluxos de potência nas linhas de interconexão devido à integração de EQEs leva a estimativas mais precisas sob um custo computacional desprezível.

\subsection{Robustez do modelo frente a erros grosseiros}

Considerando novamente o Sistema IEEE-118 com particionamento em 3 áreas (vide Fig. 6.9 e Tab. 6.3), esta última análise busca investigar o comportamento do método EEEQ na ocorrência de erros grosseiros (EGs). Para isto, será utilizado o modelo em que EQEs são incorporados como medidas convencionais (ou EEEQp), com o objetivo de verificar a viabilidade da aplicação do Teste do máximo resíduo normalizado às novas informações integradas. Ressalta-se que este procedimento também pode ser estendido para a modelagem por restrições de igualdade, analisando os multiplicadores de Lagrange normalizados.

Cinco cenários são analisados, baseando-se no plano de medição estabelecido no caso 1 da Seção 6.2, em que fluxos estão presentes em todos ramos e medidas de injeção de potência estão alocadas em barras de fronteira. Consideram-se medidas portadoras de EGs aquelas com resíduo normalizado $\left(r_{N}\right)$ maior que 3 desvios-padrão $(3 \sigma)$. No primeiro caso, um erro arbitrário de $8 \sigma$ é introduzido na medida de injeção de potência ativa alocada na barra de fronteira 19 (área 1). O segundo caso é uma extensão do primeiro, considerando também um erro de mesma magnitude na injeção de potência da barra 23, também nos limites da área 1. No terceiro cenário, avalia-se a possibilidade da deteç̧ão de erros em fluxos nos ramos de interconexão, pela introdução de erros de $10 \sigma$ nas medidas $p_{23-24}$ e $q_{19-34}$. Por fim, consideram-se EGs de $10 \sigma$ aplicados nas injeções equivalentes de potência ativa computadas para a barra 34 (caso 4) e para as barras 37 e 38 (caso $5)$.

Na Tab. (6.9) são representados os ciclos de deteç̧ão e eliminação de erros grosseiros observados no primeiro caso, listando-se os 4 maiores valores de resíduos normalizados e as medidas às quais estão associados. Neste cenário, nota-se que após a execução do estimador de estado, 


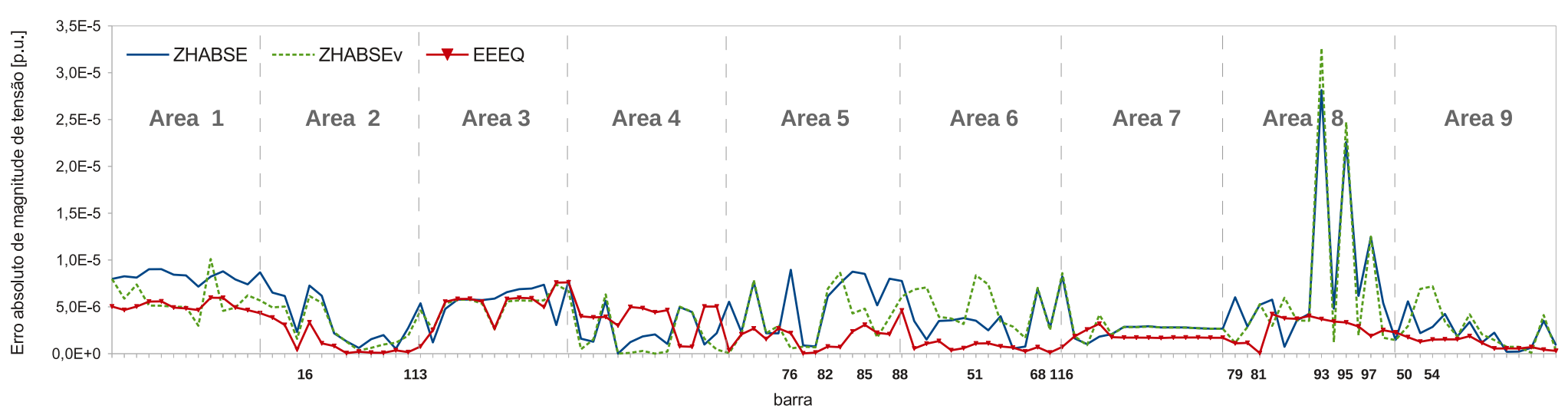

Figura 6.15 - Sistema IEEE-118 (9 áreas): erros absolutos de magnitude de tensão

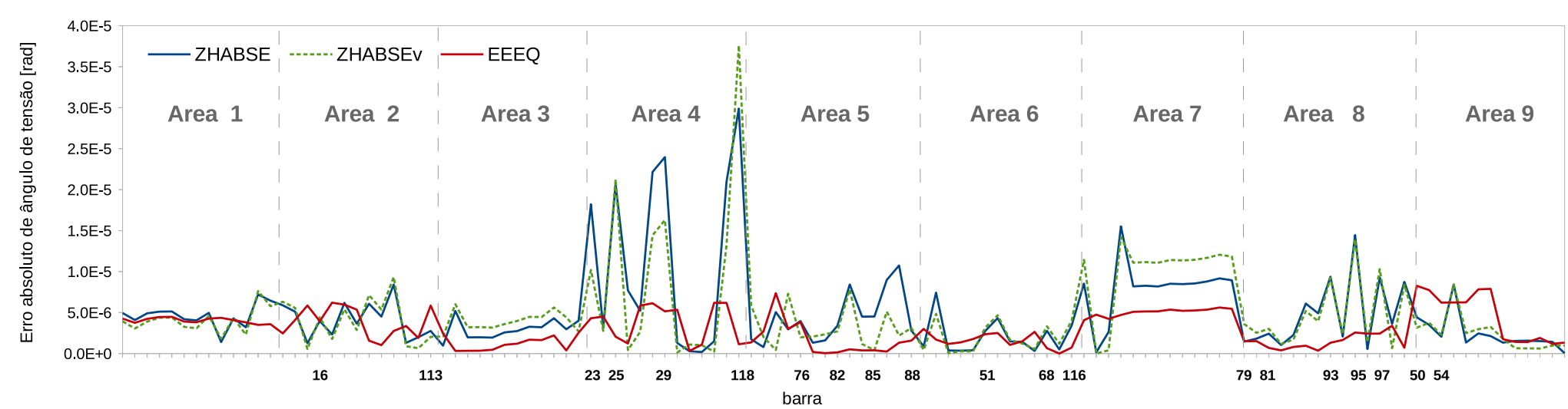

Figura 6.16 - Sistema IEEE-118 (9 áreas): erros absolutos de ângulo de tensão 
Tabela 6.9 - Maiores resíduos normalizados por ciclos de estimação e reestimação (caso 1 - erro grosseiro em $P_{19}$ )

\begin{tabular}{c|c|c|c}
\hline \hline \multicolumn{2}{c|}{ ciclo 0} & \multicolumn{2}{c}{ ciclo 1} \\
\hline tipo & $r_{N}$ & tipo & $r_{N}$ \\
\hline$P_{19}$ & 25,25 & $P_{8-5}$ & 2,45 \\
$P_{19-15}$ & 10,85 & $P_{8-9}$ & 2,42 \\
$P_{15-19}$ & 10,75 & $P_{9-8}$ & 2,36 \\
$p_{20-19}$ & 8,63 & $P_{5-8}$ & 2,16 \\
\hline
\end{tabular}

a medida de injeção $P_{19}$ é identificada como portadora de EG. Após sua eliminação e a reestimação do estado, todos os resíduos normalizados encontram-se abaixo do limiar de deteç̧ão. Comportamento similar pode ser observado na Tab. (6.10), em que a medida $P_{19}$ é identificada como errônea no primeiro ciclo de estimação, e a $P_{23}$ no segundo ciclo, de forma que dois erros simultâneos em barras de fronteira são devidamente identificados e eliminados.

Mostra-se na Tab. (6.11) que a incorporação de EQEs em tempo real não prejudica a detecção de erros grosseiros em medidas localizadas em linhas de interconexão. No referido cenário, tanto a medida de fluxo $p_{23-24}$ quanto a medida $q_{24-39}$ são identificadas e removidas do plano de medição. Um fenômeno comumente observado em métodos de EEMA baseados em dois níveis de estimação é a propagação de erros em linhas de interconexão ao estimador centralizador. Nesse caso, as estimativas obtidas na região de fronteira ficarão também comprometidas.

Finalmente, na Tabela (6.12) constata-se que um erro grosseiro na medida de injeção de potência equivalente $P_{34}^{e q}$ é devidamente identificado e removido. Este importante fato indica que, dentro da formulação de EQEs em tempo real apresentada, é possível remover dados de equivalentes que não se compatibilizam com o modelo de medição local. Em outras palavras, a incorporação de modelos equivalentes com injeções errôneas poderá ser eficazmente detectada neste contexto. No cenário 5 (Tab. 6.13), no entanto, após a remoção da injeção $P_{37}^{e q}$ e reestimação do estado, erros associados aos resíduos normalizados não são mais detectados. Nesse caso, a medida errônea $P_{38}^{e q}$ não é identificada, sugerindo a possível interatividade de erros grosseiros associados a EQEs. Ressalta-se que, neste plano de medição, as medidas disponibilizadas por PMUs não são utilizadas diretamente no estimador local. É sabido que a incorporação das mesmas pode incrementar significativamente o processo de deteç̧ão de erros (ZHAO; ABUR, 2005). Em todos os casos, sugere-se que, uma vez detectada a presença de EGs, o modelo equivalente seja prontamente removido e tenha sua consistência reavaliada. 
Tabela 6.10 - Maiores resíduos normalizados (caso 2 - erros grosseiros em $P_{19}$ e $P_{23}$ )

\begin{tabular}{c|c|c|c|c|c}
\hline \hline \multicolumn{2}{c|}{ ciclo 0} & \multicolumn{2}{c|}{ ciclo 1} & \multicolumn{2}{c}{ ciclo 2} \\
\hline tipo & $r_{N}$ & tipo & $r_{N}$ & tipo & $r_{N}$ \\
\hline$P_{19}$ & 25,26 & $P_{23}$ & 4,24 & $P_{8-5}$ & 2,45 \\
$p_{19-15}$ & 10,82 & $P_{24}^{e q}$ & 2,71 & $P_{8-9}$ & 2,42 \\
$p_{15-19}$ & 10,72 & $p_{23-25}$ & 2,67 & $P_{9-8}$ & 2,36 \\
$p_{20-19}$ & 8,76 & $p_{8-5}$ & 2,45 & $P_{5-8}$ & 2,16 \\
\hline
\end{tabular}

Tabela 6.11 - Maiores resíduos normalizados (caso 3 - erros grosseiros em $p_{23-24}$ e $q_{19-34}$ )

\begin{tabular}{c|c|c|c|c|c}
\hline \hline \multicolumn{2}{c|}{ ciclo 0} & \multicolumn{2}{c|}{ ciclo 1} & \multicolumn{2}{c}{ ciclo 2} \\
\hline tipo & $r_{N}$ & tipo & $r_{N}$ & tipo & $r_{N}$ \\
\hline$p_{23-24}$ & 74,58 & $q_{19-34}$ & 3,12 & $P_{8-5}$ & 2,45 \\
$P_{24}^{e q}$ & 62,01 & $p_{8-5}$ & 2,45 & $P_{8-9}$ & 2,42 \\
$P_{37}^{e q}$ & 10,51 & $p_{8-9}$ & 2,42 & $P_{9-8}$ & 2,36 \\
$P_{34}^{e q}$ & 10,46 & $p_{9-8}$ & 2,36 & $P_{17-15}$ & 2,17 \\
\hline
\end{tabular}

Tabela 6.12 - Maiores resíduos normalizados (caso 4 - erro grosseiro em $P_{34}^{e q}$ )

\begin{tabular}{c|c|c|c}
\hline \hline \multicolumn{2}{c|}{ ciclo 0} & \multicolumn{2}{c}{ ciclo 1} \\
\hline tipo & $r_{N}$ & tipo & $r_{N}$ \\
\hline$P_{34}^{e q}$ & 93,68 & $P_{8-5}$ & 2,45 \\
$P_{37}^{e q}$ & 93,62 & $P_{8-9}$ & 2,42 \\
$P_{38}^{e q}$ & 89,91 & $P_{9-8}$ & 2,36 \\
$p_{30-38}$ & 56,34 & $P_{17-15}$ & 2,21 \\
\hline
\end{tabular}

Tabela 6.13 - Maiores resíduos normalizados (caso 5 - erros grosseiros em $P_{37}^{e q}$ e $P_{38}^{e q}$ )

\begin{tabular}{c|c|c|c}
\hline \hline \multicolumn{2}{c|}{ ciclo 0} & \multicolumn{2}{c}{ ciclo 1} \\
\hline tipo & $r_{N}$ & tipo & $r_{N}$ \\
\hline$P_{37}^{e q}$ & 48,38 & $P_{8-5}$ & 2,45 \\
$P_{34}^{e q}$ & 48,18 & $P_{8-9}$ & 2,42 \\
$P_{38}^{e q}$ & 46,53 & $P_{9-8}$ & 2,36 \\
$p_{30-38}$ & 29,46 & $P_{17-15}$ & 2,24 \\
\hline
\end{tabular}




\section{Capítulo 7}

\section{Conclusões}

A Estimação de Estado Multiárea representa atualmente um dos campos de estudos mais férteis relacionados à modelagem de Sistemas Elétricos em tempo real, potencializada pelas crescentes tendências de descentralização da operação e pelo aumento do número de interconexões e intercâmbio de potência entre redes elétricas. É também cada vez maior a necessidade de um tratamento matemático-computacional adequado para os novos tipos de medidas popularizando-se nos SGEs, como as chamadas medidas fasoriais sincronizadas, ou sincrofasores, disponibilizadas por PMUs a taxas de até 60 amostras por segundo.

Um dos maiores desafios presentes em EEMA é a definição do modelo das redes não observáveis, externas à jurisdição local. Há uma relação de custo-benefício entre o grau de detalhamento adotado nas representações externas, a complexidade computacional e o fluxo de dados associado ao problema resultante. Nos métodos de EE Hierárquica, a ausência de tráfego de dados operacionais entre áreas permite o uso de modelos de baixa complexidade computacional, porém, resulta em estimativas de caráter subótimo, onde o refinamento do estado em barras de fronteira e a determinação de uma referência angular global passam a ser responsabilidade de um agente centralizador independente. Em Estimação de Estado Distribuída (EED) ou Descentralizada (EEDC), a existência de canais de comunicação entre áreas possibilita a obtenção de estimativas ótimas sem a etapa de coordenação, contudo a infraestrutura necessária para a operacionalização dos modelos é ainda, na prática, inexistente em muitos SEPs.

No contexto de EEDC, esta tese propõe a incorporação de Equivalentes Externos aos modelos de Estimação de Estado locais, de forma que estimativas internas confiáveis sejam obtidas sem fluxos de informações em tempo real. Embora a análise de EQEs seja tradicionalmente uma aplicação cujos modelos são definidos de forma offline, mostra-se no estudo que, a partir da consideração de medidas fasoriais sincronizadas em áreas de fronteira, é possível atualizar Equivalentes Externos, especialmente os do tipo Ward, frente a mudanças no ponto de operação do sistema, bastando para isso a alocação de PMUs em regiões de fronteira. 
O procedimento de cálculo de injeções equivalentes com base em medição fasorial sincronizada pode ser entendido como um ajuste de fronteiras em tempo real, que permite a integração de dados atualizados sobre EQEs aos próprios modelos de medição locais. Duas estratégias foram sugeridas para a incorporação das novas informações, modelando-as como pseudomedidas ou como restrições de igualdade. Esta última mostrou ser mais robusta em todos os cenários analisados. $\mathrm{Na}$ primeira, os efeitos da escolha de pesos incorretos foram constatados, onde desvios significativos em relação à referência foram detectados quando variâncias maiores foram utilizadas. Em ambas as formulações, técnicas tradicionais de análise de observabilidade, estimação de estado e processamento de erros grosseiros são totalmente compatíveis.

A partir das simulações computacionais efetuadas, os seguintes pontos podem ainda ser considerados. Primeiramente, estimativas de fronteira obtidas considerando Equivalentes Externos em tempo real mostraram ser superiores ou compatíveis (em termos de precisão) às obtidas pelos métodos hierárquicos de duas etapas. A supressão da etapa posterior de centralização resulta em ganhos computacionais expressivos, eliminando também eventuais problemas relacionados a dimensões elevadas no modelo, particularmente existentes em métodos de EEH. Os resultados indicam também que EQEs Ward calculados em tempo real atuam como medidas de compensação no problema de EEMA, minimizando desvios em áreas de interconexão e permitindo que estado global seja obtido de forma descentralizada. É possível constatar que o desempenho superior em termos de precisão não se deve exclusivamente ao uso de medidas fasoriais sincronizadas para o cálculo de EQEs, na medida que a incorporação destas aos métodos de duas etapas não resultou em desempenho semelhante.

Para a dedução de EQEs em tempo real, considera-se na metodologia proposta que PMUs estejam alocados em barras de fronteira e que informações sobre a topologia externa estejam disponíveis. A primeira restrição é perfeitamente factível, na medida em que grande parte das PMUs incorporadas às redes elétricas são utilizadas para o monitoramento de oscilações interárea e na supervisão e proteção de linhas de interconexão. Unidades de medição fasorial podem também fornecer as referências angulares das subáreas, resolvendo a questão do assincronismo em EEMA. Sobre a segunda restrição, é possível constatar que, como a frequência de alterações topológicas é normalmente muito inferior a mudanças no ponto de operação, certas variáveis podem ser facilmente disponibilizadas entre as subáreas por meio de canais de comunicação simples, considerando, por exemplo, informações de status de chaves, disjuntores e, eventualmente, a localização de grandes fontes reativas. Adicionalmente, verifica-se que em EQEs do tipo Ward, o efeito de erros em parâmetros externos é anulado desde que as injeções sejam calculadas no ponto de operação atual do sistema.

Os testes efetuados demonstram também que a característica de detecção de medidas errôneas na interconexão (fluxos e injeções), desejável em modelos de EEMA, é mantida na formulação proposta. Adicionalmente, a presença de erros grosseiros em injeções equivalentes pode igualmente 
ser detectada, permitindo que EQEs Ward não compatíveis com o modelo de medição local sejam rejeitados em tempo real. Sugere-se que, diante de erros nos equivalentes ou da indisponibilidade de PMUs, EQEs Ward sejam processadas da forma convencional, isto é, considerando o estado fornecido pelo estimador interno. Nesta situação, a sincronização dos sistemas, quando necessária, pode ser efetuada utilizando métodos clássicos de EEMA.

Por fim, esta tese traz importantes desdobramentos ao contexto específico de EQEs. Métodos fundamentados no modelo Ward consideram, para o cálculo das injeções equivalentes, os estados das barras de fronteira determinados pelo estimador interno. Visto que, a priori, somente metodologias em EEMA com elevados custos computacionais fornecem estimativas ótimas em nós de fronteira, a determinação de EQEs confiáveis sem se recorrer a tais métodos é improvável. Nesse sentido, os modelos desenvolvidos permitem, de forma simples, a obtenção de estimativas precisas nestas regiões, fornecendo informações confiáveis para a determinação de EQEs e, consequentemente, para as demais aplicações que necessitam de representações externas, como as relacionadas à Análise de Segurança.

Em suma, as maiores contribuições do trabalho ao campo de estudo são citadas a seguir:

- Tratamento integrado de Equivalentes Externos (EQEs) e Estimação de Estado (EE), utilizando medição fasorial sincronizada;

- Uma metodologia de EEMA onde EQEs são utilizados para incrementar a precisão do estado interno, de forma que uma etapa de centralização não é mais necessária;

- Modelos operam sem fluxos de dados em tempo real (somente dados da topologia externa) e possuem elevado desempenho computacional;

- Compatibilidade com métodos clássicos de EE, Análise de Observabilidade e Processamento de Erros grosseiros;

- Possibilidade da rejeição em tempo real de representações equivalentes não compatíveis com os modelos de medição locais;

- Sincronização de distintas referências angulares pode ser efetuada diretamente por PMUs;

- EQEs computados em tempo real com precisão e confiabilidade para uso posterior em aplicações de Análise de Segurança.

No que segue, listam-se alguns pontos da pesquisa passíveis de serem investigados e aperfeiçoados em trabalhos futuros:

- Análise dos modelos de EQEs incorporados considerando métodos de EE mais robustos, utilizando transformação ortogonal ou matriz Aumentada; 
- Formalizar a estratégia para alocação de PMUs considerando o conceito de níveis de não observabilidade (NUQUI; PHADKE, 2005);

- Tratamento de condições relacionadas aos limites operacionais de PMUs, como capacidade do canal e do concentrador de dados;

- Uso de medidas fasoriais nos estimadores locais e estudo de confiabilidade das medidas;

- Avaliação da correlação entre medidas do sistema SCADA e SMFS;

- Formulação de EQEs em tempo real no contexto da Estimação de Estado Generalizada;

- Investigação e modelagem da interatividade de erros grosseiros relacionados a EQEs;

- Descentralização da metodologia proposta a nível de Subestação e análise no contexto de sistemas de distribuição.

- Avaliação dos modelos frente a contingências, no contexto de Análise de Segurança; 


\section{Referências}

ABOYTES, F.; CORY, B. Identification of measurement, parameter and configuration errors in static state estimation. Proc. of the 9th PICA Conf., 1975. New Orleans, p. 298 - 302, June 1975.

AHMED, S.; BRAMELLER, A. New algorithm for diakoptical static state estimation. Generation, Transmission and Distribution, IEE Proceedings C, 1991. v. 138, n. 3, p. 185 -192, 1991.

ALLEMONG, J.; RADU, L.; SASSON, A. A fast and reliable state estimation algorithm for AEP's new control center. Power Apparatus and Systems, IEEE Transactions on, 1982. PAS-101, n. 4, p. 933-944, 1982. ISSN 0018-9510.

AMERONGEN, R. A. M. V. A general-purpose version of the fast decoupled load flow. Power Systems, IEEE Transactions on, 1989. v. 4, n. 2, p. 760-770, 1989. ISSN 0885-8950.

ÂNGELOS, E. W. S.; ASADA, E. N. Multi-area state estimation with PMU and external system modeling. In: Power and Energy Society General Meeting, 2012 IEEE. San Diego CA: IEEE, 2012. p. 1-7. ISSN 1944-9925.

ASCHMOneIT, F. C.; PETERSOn, N. M.; ADRIAN, E. C. State estimation with equality constraints. Tenth PICA Conference Proceedings, 1977. p. 427 - 430, 1977.

BALDWIN, T.; MILI, L.; BOISEN M.B., J.; ADAPA, R. Power system observability with minimal phasor measurement placement. Power Systems, IEEE Transactions on, 1993. v. 8, p. 707 $-715,1993$.

BEZ, D.; SIMÕES-COSTA, A. Inclusão de medidas fasoriais na modelagem em tempo real de sistemas de potência via estimação de estados em dois estágios. In: IV Simpósio Brasileiro de Sistemas Elétricos (SBSE). Goiania GO: SBSE, 2012. p. 1-6.

BOOST. Boost C ++ Libraries. 2013. Disponível em: <http://www.boost.org/>.

BOSE, A. Modeling of external networks for on-line security analysis. Power Engineering Review, IEEE, 1984. PER-4, n. 8, p. 48-49, 1984. ISSN 0272-1724.

New computer applications for system operations using phasor measurements. In: Power and Energy Society General Meeting, 2012 IEEE. San Diego CA: IEEE, 2012. p. 1-5. ISSN 19449925.

BOSE, A.; CLEMENTS, K. Real-time modeling of power networks. Proceedings of the IEEE, 1987. v. 75 , n. 12 , p. 1607-1622, 1987. ISSN 0018-9219. 
CARO, E.; CONEJO, A.; MINGUEZ, R. Decentralized state estimation and bad measurement identification: An efficient lagrangian relaxation approach. Power Systems, IEEE Transactions on, 2011. v. 26, n. 4, p. 2500-2508, 2011. ISSN 0885-8950.

CHAKRABARTI, S.; KYRIAKIDES, E.; LEDWICH, G.; GHOSH, A. A comparative study of the methods of inclusion of PMU current phasor measurements in a hybrid state estimator. In: Power and Energy Society General Meeting, 2010 IEEE. Minneapolis: IEEE, 2010. p. 1-7.

CLEMENTS, K.; DAVIS, P. Multiple bad data detectability and identifiability: A geometric approach. Power Delivery, IEEE Transactions on, 1986. v. 1, n. 3, p. 355-360, 1986. ISSN 08858977.

CONEJO, A.; TORRE, S. D. L.; CANAS, M. An optimization approach to multiarea state estimation. Power Systems, IEEE Transactions on, 2007. v. 22, n. 1, p. 213-221, 2007. ISSN 0885-8950.

DE ALMEIDA, M.; ASADA, E.; GARCIA, A. Power system observability analysis based on gram matrix and minimum norm solution. Power Systems, IEEE Transactions on, 2008. v. 23, n. 4, p. 1611-1618, 2008. ISSN 0885-8950.

DE ALMEIDA, M.; GARCIA, A.; ASADA, E. Regularized least squares power system state estimation. Power Systems, IEEE Transactions on, 2012. v. 27, n. 1, p. 290-297, 2012. ISSN 0885-8950.

DECKMAnN, S.; PIZZOLANTE, A.; MONTICELli, A.; STOTT, B.; ALSAC, O. Numerical testing of power system load flow equivalents. Power Apparatus and Systems, IEEE Transactions on, 1980. PAS-99, n. 6, p. 2292-2300, 1980. ISSN 0018-9510.

Studies on power system load flow equivalencing. Power Apparatus and Systems, IEEE Transactions on, 1980. PAS-99, n. 6, p. 2301-2310, 1980. ISSN 0018-9510.

DO COUTTO FILHO, M. B.; SOUZA, J. C. S. D. Forecasting-aided state estimation - part i: Panorama. Power Systems, IEEE Transactions on, 2009. v. 24, n. 4, p. 1667-1677, 2009. ISSN 0885-8950.

DO COUTTO FILHO, M. B.; SOUZA, J. C. S. D.; FREUND, R. Forecasting-aided state estimation - part ii: Implementation. Power Systems, IEEE Transactions on, 2009. v. 24, n. 4, p. 1678-1685, 2009. ISSN 0885-8950.

DURAN, H.; ARVANITIDIS, N. Simplifications for area security analysis: A new look at equivalence. Power Apparatus and Systems, IEEE Transactions on, 1972. PAS-91, n. 2, p. 670-679, 1972. ISSN 0018-9510.

EBRAHIMIAN, R.; BALDICK, R. State estimation distributed processing. Power Systems, IEEE Transactions on, 2000. v. 15, p. $1240-1246,2000$.

ECLIPSE. Eclipse IDE for $C / C++$ Developers. 2013. Disponível em: <http://www.eclipse.org$1>$. 
EL-FATTAH, Y. M.; RIBBENS-PAVELLA, M. Multi-level approach to state estimation for electric power systems. In: Proc. 4th IFAC Symp. Identification \& Syst. Parameter Estimation. Tbilisi: [s.n.], 1976. p. Paper 6.1.

FALCAO, D.; WU, F.; MURPHY, L. Parallel and distributed state estimation. Power Systems, IEEE Transactions on, 1995. p. $724-730,1995$.

GARCIA, A.; MONTICELlI, A.; ABREU, P. Fast decoupled state estimation and bad data processing. Power Apparatus and Systems, IEEE Transactions on, 1979. PAS-98, n. 5, p. 16451652, 1979. ISSN 0018-9510.

GEISLER, K.; BOSE, A. State estimation based external network solution for on-line security analysis. Power Apparatus and Systems, IEEE Transactions on, 1983. PAS-102, p. 2447 -2454, 1983.

GJELSVIK, A.; AAM, S.; HOLTEN, L. Hachtel's augmented matrix method - a rapid method improving numerical stability in power system static state estimation. Power Apparatus and Systems, IEEE Transactions on, 1985. PAS-104, n. 11, p. 2987-2993, 1985. ISSN 0018-9510.

GOMEZ-EXPOSITO, A.; JAEN, A. de la V.; GOMEZ-QUILES, C.; ROUSSEAUX, P.; CUTSEM, T. V. A taxonomy of multi-area state estimation methods. Electric Power Systems Research, 2011. v. 81, n. 4, p. 1060-1069, 2011.

HABIBALLAH, I. Modified two-level state estimation approach. Generation, Transmission and Distribution, IEE Proceedings-, 1996. v. 143, p. 193 -199, 1996.

HORISBERGER, H. P.; RICHARD, J. C.; ROSSIER, C. A fast decoupled static state-estimator for electric power systems. Power Apparatus and Systems, IEEE Transactions on, 1976. v. 95, n. 1, p. 208-215, 1976. ISSN 0018-9510.

HSL. The HSL Mathematical Software Library. 2013. Disponível em: < http://www.hsl.rl.ac.uk/index.html>.

IEEE STD C37.118-2005. IEEE Standard for Synchrophasors for Power Systems (Revision of IEEE Std 1344-1995). 2005.

IEEE STD C37.118.1-2011. IEEE Standard for Synchrophasors for Power Systems (Revision of IEEE Std C37.118-2005). 2011.

IEEE STD C37.118.2-2011. IEEE Standard for Synchrophasor Data Transfer for Power Systems (Revision of IEEE Std C37.118-2005). 2011. 1-53 p.

IRVING, M. R.; STERLING, M. J. M. Multi-level state estimation and optimal dispatch techniques. in Proc. IFAC Symp. Computer Applications in Large-Scale Power Systems, 1979. p. 117-125, 1979.

JIANG, W.; VITTAL, V.; HEYDT, G. A distributed state estimator utilizing synchronized phasor measurements. Power Systems, IEEE Transactions on, 2007. v. 22, n. 2, p. 563 -571, 2007.

Diakoptic state estimation using phasor measurement units. Power Systems, IEEE Transactions on, 2008. v. 23 , n. 4, p. $1580-1589,2008$. 
KORRES, G. A distributed multiarea state estimation. Power Systems, IEEE Transactions on, 2011. v. 26, n. 1, p. $73-84,2011$.

KRUMPhOlZ, G. R.; CLEMENTS, K.; DAVIS, P. Power system observability: A practical algorithm using network topology. Power Apparatus and Systems, IEEE Transactions on, 1980. PAS-99, n. 4, p. 1534-1542, 1980. ISSN 0018-9510.

KURZYN, M. S. Real-time state estimation for large-scale power systems. Power Apparatus and Systems, IEEE Transactions on, 1983. PAS-102, n. 7, p. 2055-2063, 1983. ISSN 0018-9510.

LAKSHMINARASIMHAN, S.; GIRGIS, A. A. Hierarchical state estimation applied to wide-area power systems. Power Engineering Society General Meeting, 200\%. IEEE, 2007. p. 1 -6, 2007.

LIN, H.; SAMBAMOORTHY, S.; SHUKLA, S.; THORP, J.; MILI, L. A study of communication and power system infrastructure interdependence on PMU-based wide area monitoring and protection. In: Power and Energy Society General Meeting, 2012 IEEE. San Diego: IEEE, 2012. p. 1-7. ISSN 1944-9925.

LO, K.; SALEM, M. M.; MCCOLL, R. D.; MOFFATT, A. M. Two-level state estimation for large power system. I. Algorithms. Generation, Transmission and Distribution, IEE Proceedings C, 1988. v. 135 , n. 4 , p. 299-308, 1988. ISSN 0143-7046.

. Two-level state estimation for large power system. II. Computational experience. Generation, Transmission and Distribution, IEE Proceedings C, 1988. v. 135, n. 4, p. 309-318, 1988. ISSN 0143-7046.

LONDON, J.; ALBERTO, L.; BRETAS, N. Analysis of measurement-set qualitative characteristics for state-estimation purposes. Generation, Transmission Distribution, IET, 2007. v. 1, n. 1, p. 39-45, 2007. ISSN 1751-8687.

MONTICELLI, A.; DECKMANN, S.; GARCIA, A.; STOTT, B. Real-time external equivalents for static security analysis. Power Apparatus and Systems, IEEE Transactions on, 1979. PAS-98, n. 2, p. $498-508,1979$. ISSN 0018-9510.

MONTICELLI, A.; GARCIA, A. Reliable bad data processing for real-time state estimation. Power Apparatus and Systems, IEEE Transactions on, 1983. PAS-102, n. 5, p. 1126-1139, 1983. ISSN 0018-9510.

. Fast decoupled state estimators. Power Systems, IEEE Transactions on, 1990. v. 5, n. 2, p. 556-564, 1990. ISSN 0885-8950.

MONTICELLI, A.; WU, F. A method that combines internal state estimation and external network modeling. Power Apparatus and Systems, IEEE Transactions on, 1985. PAS-104, p. 91 $-103,1985$.

Network observability: Identification of observable islands and measurement placement. Power Apparatus and Systems, IEEE Transactions on, 1985. PAS-104, n. 5, p. 1035-1041, 1985. ISSN 0018-9510.

MONTICELLI, A. J. State Estimation in Electric Power Systems: A Generalized Approach. Boston: Kluwer Academic Publishers, 1999. 
MORAES, R.; VOLSKIS, H.; HU, Y. Deploying a large-scale PMU system for the brazilian interconnected power system. Electric Utility Deregulation and Restructuring and Power Technologies, 2008. DRPT 2008. Third International Conference on, 2008. p. 143 -149, 2008.

NASPI. North American SynchroPhasor Iniative (NASPI). 2011. Disponível em: < http://www.naspi.org $>$.

NUQUI, R.; PHADKE, A. Phasor measurement unit placement techniques for complete and incomplete observability. Power Delivery, IEEE Transactions on, 2005. v. 20, n. 4, p. 2381-2388, 2005. ISSN 0885-8977.

OPENMP. The OpenMP API specification for parallel programming. 2013. Disponível em: < http://openmp.org/wp/>. Acesso em: 8 Out. 2013.

PATEL, M.; GIRGIS, A. Two-level state estimation for multi-area power system. Power Engineering Society General Meeting, 200\%. IEEE, 2007. p. 1 -6, 2007.

PHADKE, A. Synchronized phasor measurements in power systems. Computer Applications in Power, IEEE, 1993. v. 6, p. $10-15$, 1993. ISSN 2.

PHADKE, A.; THORP, J. Synchronized Phasor Measurements and Their Applications. Boston, MA: Springer Science+Business Media, LLC, 2008.

PHADKE, A. G.; THORP, J. S.; KARIMI, K. J. State estimation with phasor measurements. Power Systems, IEEE Transactions on, 1986. p. 233 -238, 1986.

QUiNTANA, V.; SIMÕES-COSTA, A.; MANDEL, A. Power system topological observability using a direct graph-theoretic approach. Power Apparatus and Systems, IEEE Transactions on, 1982. PAS-101, n. 3, p. 617-626, 1982. ISSN 0018-9510.

RICE, M.; HEYDT, G. Power systems state estimation accuracy enhancement through the use of PMU measurements. Transmission and Distribution Conference and Exhibition, 2005/2006 IEEE PES, 2006. p. $161-165,2006$.

SASAKI, H.; AOKI, K.; YOKOYAMA, R. A parallel computation algorithm for static state estimation by means of matrix inversion lemma. Power Systems, IEEE Transactions on, 1987. v. 2 , p. $624-631,1987$.

SCHWEPPE, F. Power system static-state estimation, part iii: Implementation. Power Apparatus and Systems, IEEE Transactions on, 1970. PAS-89, p. $130-135,1970$.

SCHWEPPE, F.; ROM, D. Power system static-state estimation, part ii: Approximate model. Power Apparatus and Systems, IEEE Transactions on, 1970. p. 125 -130, jan. 1970.

SCHWEPPE, F.; WILDES, J. Power system static-state estimation, part i: Exact model. Power Apparatus and Systems, IEEE Transactions on, 1970. p. 120 -125, jan. 1970.

SEIDU, K.; MUKAI, H. Parallel multi-area state estimation. Power Apparatus and Systems, IEEE Transactions on, 1985. PAS-104, n. 5, p. 1025 -1034, 1985. 
SIMÕES-COSTA, A.; ALBUQUERQUE, A.; BEZ, D. An estimation fusion method for including phasor measurements into power system real-time modeling. Power Systems, IEEE Transactions on, 2013. v. 28, n. 2, p. 1910-1920, 2013. ISSN 0885-8950.

SIMÕES-COSTA, A.; QUINTANA, V. A robust numerical technique for power system state estimation. Power Apparatus and Systems, IEEE Transactions on, 1981. PAS-100, n. 2, p. 691698, 1981. ISSN 0018-9510.

An orthogonal row processing algorithm for power system sequential state estimation. Power Apparatus and Systems, IEEE Transactions on, 1981. PAS-100, n. 8, p. 3791-3800, 1981. ISSN 0018-9510.

STOTT, B.; ALSAC, O. Fast decoupled load flow. Power Apparatus and Systems, IEEE Transactions on, 1974. PAS-93, n. 3, p. 859-869, 1974. ISSN 0018-9510.

STOTT, B.; ALSAC, O.; MONTICELLI, A. Security analysis and optimization. Proceedings of the IEEE, 1987. v. 75, p. 1623 - 1644, 1987. ISSN 0018-9219.

STOVALL, J.; KIRBY, B.; OVERBYE, T.; THORP, J.; PHADKE, A. Issues associated with the development of a wide-area analysis and environment. System Sciences, 2006. HICSS 06. Proceedings of the 39th Annual Hawaii International Conference on, 2006. p. 240a, 2006.

THORP, J.; PHADKE, A.; KARIMI, K. Real time voltage-phasor measurement for static state estimation. Power Apparatus and Systems, IEEE Transactions on, 1985. PAS-104, p. 3098 -3106, 1985. ISSN 11.

U.S. DEPARTMENT OF ENERGY. Final Report on the August 14'2003 Blackout in the United States and Canada: Causes and Recommendations. Canada, 2004.

UWEE. University of Washington Electrical Engineering: Power system test cases archive. 2011. Disponível em: <http://www.ee.washington.edu/research/pstca/>.

VAN CUTSEM, T.; HORWARD, J.; RIBBENS-PAVELLA, M. A two-level static state estimator for electric power systems. Power Apparatus and Systems, IEEE Transactions on, 1981. PAS-100, p. $3722-3732,1981$.

VAN CUTSEM, T.; HORWARD, J. L.; RIBBENS-PAVELLA, M.; EL-FATTAH, Y. M. Hierarchical state estimation. International Journal of Electrical Power \& Energy Systems, 1980. v. 2, p. $70-80,1980$.

VAN CUTSEM, T.; RIBBENS-PAVELLA, M. Critical survey of hierarchical methods for state estimation of electric power systems. Power Apparatus and Systems, IEEE Transactions on, 1983. PAS-102, p. $3415-3424,1983$.

WALLACH, Y.; HANDSCHIN, E.; BONGERS, C. An efficient parallel processing method for power system state estimation. Power Engineering Review, IEEE, 1981. PER-1, n. 11, p. 20 -21, 1981.

WARD, J. B. Equivalent circuits for power-flow studies. American Institute of Electrical Engineers, Transactions of the, 1949. v. 68, n. 1, p. 373 -382, 1949. ISSN 0096-3860. 
WU, F.; MONTICELLI, A. Critical review of external network modelling for online security analysis. International Journal of Electrical Power \& Energy Systems, 1983. v. 5, p. 222 - 235, 1983.

ZHAO, H. A new state estimation model of utilizing PMU measurements. Power System Technology, 2006. PowerCon 2006. International Conference on, 2006. p. 1 -5, 2006.

ZHAO, H.; GUO, J.; FAN, X.-d. The study of distributed multi-area state estimation algorithm. Power System Technology and IEEE Power India Conference, 2008. POWERCON 2008. Joint International Conference on, 2008. p. 1 -5, 2008.

ZHAO, L.; ABUR, A. Multi area state estimation using synchronized phasor measurements. Power Systems, IEEE Transactions on, 2005. v. 20, p. 611 - 617, 2005.

ZHOU, M. Advanced system monitoring with phasor measurements. Ph. D. Dissertation, Virginia Tech, 2008. 2008.

ZHOU, M.; CENTENO, V.; THORP, J.; PHADKE, A. An alternative for including phasor measurements in state estimators. Power Systems, IEEE Transactions on, 2006. v. 21, p. 1930 $-1937,2006$. ISSN 4.

ZHU, J.; ABUR, A. Effect of phasor measurements on the choice of reference bus for state estimation. Power Engineering Society General Meeting, 200\%. IEEE, 2007. p. 1 -5, 2007.

ZIVANOVIC, R.; CAIRNS, C. Implementation of PMU technology in state estimation: an overview. AFRICON, 1996., IEEE AFRICON 4th, 1996. v. 2, p. $1006-1011$ vol.2, 1996. 